\title{
The Journal of THORACIC AND \\ CARDIOVASCULAR SURGERY
}

Copyright $(1993$ by Mosby-Year Book, Inc.

Volume 105, Number 4

\section{Contents April 1993}

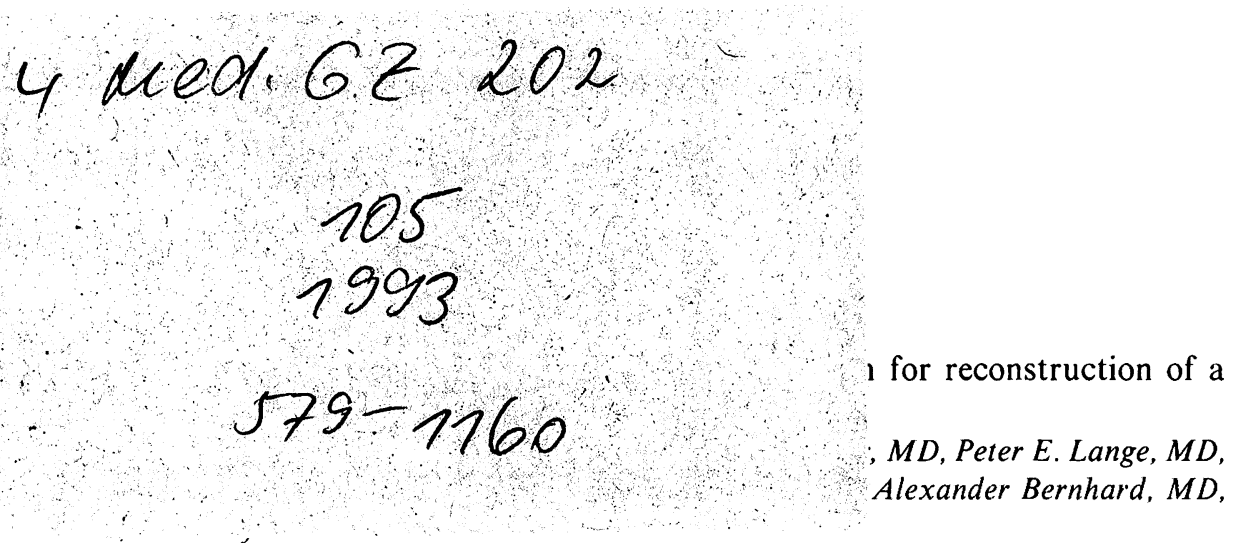

The functional behavior of monocuspid and bicuspid patches made from glutaraldehydetreated porcine aortic roots for experimental repair of a surgically created hypoplastic pulmonary root was investigated. The function of the bicuspid design is superior to that of the monocuspid design and permits the construction of a competent and stenosis-free valve mechanism.

Intraventricular repair for Taussig-Bing anomaly

Yasunaru Kawashima, MD, Hikaru Matsuda, MD (by invitation), Toshikatu Yagihara, MD (by invitation), Yasuhisa Shimazaki, MD (by invitation), Fumio Yamamoto, MD (by invitation), Kyoichi Nishigaki, MD (by invitation), Takuya Miura, MD (by invitation), and Hideki Uemura, MD (by invitation), Osaka, Japan

Ten patients with Taussig-Bing anomaly, mostly with a side-by-side relationship of the great arteries, underwent satisfactory intraventricular rerouting with no deaths and without late development of subaortic obstruction. This operation appears to be the method of choice for this subset of patients when care is paid to create an unobstructed left ventricular-aortic route during the operation.

Vol. 105, No. 4, April 1993. The Journal of Thoracic and Cardiovascular Surgery (ISSN 0022-5223) is published monthly (six issues per volume, two volumes per year) by Mosby-Year Book, Inc., 11830 Westline Industrial Dr., St. Louis, MO 63146-3318. Second-class postage paid at St. Louis, Mo., and additional mailing. offices. POSTMASTER: Send change of address to: The Journal of Thoracic and Cardiovascular Surgery, Mosby, 11830 Westline Industrial Dr., St. Louis, MO 63146$3318,(800)$ 325-4177, ext. 4351, or (314) 453-4351. Annual subscription rates for 1993: domestic, \$112.00 for individuals and \$192.00 for institutions. Printed in the U.S.A. Copyright $\mathcal{c} 1993$ by Mosby-Year Book, Inc. All rights reserved. No part of this publication may be reproduced, stored, or transmitted in any form or by any means, electronic or mechanical including photocopy, recording, or any information storage and retrieval system, without permission in writing from the publisher. 


\section{Contents continued}

A computer simulation of the plasma leakage through a vascular prosthesis made of expanded polytetrafluoroethylene

Ryoko Tabata, MD, Takayuki Kobayashi, PhD, Atsumi Mori, MD, Shuichi Matsuno, MD, Shoji Watarida, MD, Masahiko Onoe, MD, Takaaki Sugita, MD, Shoichirou Shiraisi, MD, and Takehisa Nojima, MD, Shiga, Japan

Computer simulation disclosed that the plasma leakage through the wall of an ePTFE vascular prosthesis depends on inn r pressure, surface tension, and a mean distance between PTFE fibers in the direction olntveraitataren ial axis of the vascular prosthesis.

\section{Bibliotnek \\ München}

\section{Surgery for Acquired Heart Disease}

The effect of coronary reoperation on the survival of patients with stenoses in saphe-

nous vein bypass grafts to coronary arteries

Bruce W. Lytle, MD, Floyd D. Loop, MD, Paul C. Taylor, MD (by invitation), Marlene Goormastic, MPH (by invitation), Robert W. Stewart, MD (by invitation), Roberto Novoa, MD (by invitation), Patrick McCarthy, MD (by invitation), and Delos M. Cosgrove, MD, Cleveland, Ohio. With the technical assistance of Maura J. Schnauffer

Patients with late stenoses ( $\geq 5$ years after operation) in SV grafts to coronary arteries have better survival with early reoperation than with initial medical treatment.

The right gastroepiploic artery graft: Clinical and angiographic midterm results in 200 patients

Hisayoshi Suma, MD (by invitation), Yasuhiko Wanibuchi, MD (by invitation), Yasushi Terada, MD (by invitation), Sachito Fukuda, MD (by invitation), Tetsuro Takayama, $M D$ (by invitation), and Shoichi Furuta, MD (by invitation), Tokyo, Japan

The GEA was used for CABG in 200 patients, with 6 early and 4 late deaths. Follow-up ( 6 to 70 months, mean 27 months) showed a GEA patency rate of $95 \%$ at both early (mean 2 months) and late (mean 2 years) angiography. Stress scintigraphy revealed satisfactory GEA function.

Continued on page $8 A$

\begin{tabular}{|c|c|c|c|c|c|c|}
\hline \multirow{2}{*}{$\begin{array}{l}1993 \text { Annual } \\
\text { subscription rates }\end{array}$} & \multirow[b]{2}{*}{ U.S.A. } & \multicolumn{2}{|c|}{ International surface mail } & \multicolumn{3}{|c|}{ International airmail } \\
\hline & & All regions (except Canada)* & Canadat & Canadat & Mexico & All other \\
\hline Institutions $\ddagger$ & $\$ 192.00$ & $\$ 222.00$ & $\$ 235.44$ & $\$ 265.44$ & $\$ 252.00$ & $\$ 262.00$ \\
\hline Students, residents§ & $\$ 51.00$ & $\$ 81.00$ & $\$ 84.57$ & $\$ 114.57$ & $\$ 111.00$ & $\$ 121.00$ \\
\hline
\end{tabular}

Subscription rates include supplements. Single copies are $\$ 10.00$. Remittances should be made by check, draft, post office or express money order, in U.S. funds, drawn through a U.S. bank, payable to this JouRNAL. Claims for missing issues will be serviced only within 6 months of cover date. Single copy prices will be charged on missing issues older than 6 months from cover date. Back issues generally are available for the previous 5 years. Contact the publisher to confirm availability of specific issues.

*Contact exclusive agents in Japan and India for subscription information. Igaku-Shoin Foreign Publications Department, 1-28-36 Hongo, Bunkyo-ku, Tokyo, 113 Japan. Universal Subscription Agency Pvt. Ltd., 18-19 Community Centre, Saket P. B. No. 8, New Delhi 110017, India.

†Canadian 7\% Goods and Services Tax, calculated on U.S. base rate, has been added and will be paid by Mosby to Revenue Canada under GST No. R 127341295.

‡Institutional (multiple-reader) subscriptions are available to public and private libraries, schools, hospitals, and clinics; city, county, state, provincial, and national government bureaus and departments; and all commercial and private institutions and organizations.

§Individual subscriptions and all student-rate subscriptions must be in the names of, billed to, and paid by individuals. All student-rate requests must indicate training status and name of institution.

Subscriptions may begin at any time. Contact Mosby, Subscription Services, 11830 Westline Industrial Dr., St. Louis, MO 63146-3318. U.S.A.: phone (800) $325-4177$, ext. 4351 , or (314) $453-4351$.

Statements and opinions expressed in the articles and communications herein are those of the author(s) and not necessarily those of the Editor or publisher, and the Editor and publisher disclaim any responsibility or liability for such material. Neither the Editor nor the publisher guarantees, warrants, or endorses any product or service advertised in this publication, nor do they guarantee any claim made by the manufacturer of such product or service. 


\section{Volume 105 Number $4 \quad$ April 1993 \\ The Journal of THORACIC AND CARDIOVASCULAR SURGERY}

\section{IN MEMORIAM}

Dr. E. Stanley Crawford died in Houston, Texas, on October 27, 1992. Dr. Charles Dubost, in attendance a few years ago at a surgical meeting in the United States, said that his real reason for coming to America was to pay his usual visit to "the greatest surgeon in your country." When pressed for his name, Dubost said, "Stanley Crawford."

Born in Evergreen, Alabama, on May 12, 1922, Stanley Crawford was a brilliant cardiovascular surgeon whose workload throughout his life was staggering, in spite of which his results were exemplary and set a standard for the rest of us to follow. While his early and richly rewarding writings stressed the drama of the domain in which he worked, in his later years his publications became precise and analytical. They too, in a different way, were richly rewarding to their readers. The perceptible transition was just one of the many remarkable and totally admirable characteristics of this lovable, gifted man. The readership of THE Journal of Thoracic and Cardiovascular Surgery surely joins the Editor in his loneliness for this productive, provocative, and genuinely unique and wonderful surgeon. 


\section{Hemostatic activation during cardiopulmonary bypass with different aprotinin dosages in pediatric patients having cardiac operations}

The effect of high-dose aprotinin treatment on hemostatic activation during cardiopulmonary bypass in pediatric patients having cardiac operations was investigated. Sixty patients weighing less than $10 \mathrm{~kg}$ undergoing cardiac operations for different types of congenital heart diseases were studied: 20 patients were treated with aprotinin $2 \times 15,000 \mathrm{KIU} / \mathrm{kg}, 20$ patients with $2 \times 30,000 \mathrm{KIU} / \mathrm{kg}$, and 20 patients without aprotinin treatment served as the control group. Different split products of fibrinogen and/or fibrin and the fibrinolytic activity on fibrin plates were measured to assess fibrinolytic activation. F1/F2 prothrombin fragments, thrombin-antithrombin III-complex, and fibrin monomers were measured to estimate thrombin activation. There was a significant dose-dependent reduction in fibrin-fibrinogen split product formation during cardiopulmonary bypass: In the high-dose aprotinin group the concentration of the split products at the end of bypass was $1.5 \pm 0.6 \mu \mathrm{g} / \mathrm{ml}$, compared with $3.4 \pm 3.0 \mu \mathrm{g} / \mathrm{ml}$ in the low-dose aprotinin group and $6.7 \pm 3.5 \mu \mathrm{g} / \mathrm{ml}$ in the control group $(p<00.5)$. Fibrinolytic activation on fibrin plates was also significantly reduced by aprotinin. Fibrin monomer formation was significantly diminished at the end of cardiopulmonary bypass in the high-dose group: $9.2 \pm 5.2 \mu \mathrm{g} / \mathrm{ml}$ compared with $21.6 \pm 14 \mu \mathrm{g} / \mathrm{ml}$ in the control group $(p<00.5)$. Elastase in complex with $\alpha_{1}$-protease inhibitor at the end of bypase was increased to the same amount in the three groups: $784 \pm 278 \mathrm{ng} / \mathbf{m L}$ (control group), $693 \pm 189 \mathrm{ng} / \mathrm{ml}$ (low-dose aprotinin), and $719 \pm 270 \mathrm{ng} / \mathrm{mL}$ (high dose aprotinin) (no significant difference). Blood loss 6 hours postoperatively was significantly $(p<00.5)$ less in the high-dose group $\left(99 \pm 32 \mathrm{ml} / \mathrm{m}^{2}\right)$ than in the control group $\left(164 \pm 87 \mathrm{ml} / \mathrm{m}^{2}\right.$; low-dose group: $\left.160 \pm 106 \mathrm{ml} / \mathrm{m}^{2}\right)$. These observations suggest an attenuation of hemostatic activation during cardiopulmonary bypass with less plasmin formation and, because of inhibition of contact activation, less thrombin generation with aprotinin treatment. Thus the thrombotic-thrombolytic equilibrium is kept more balanced after cardiopulmonary bypass. High-dose aprotinin treatment is recommended for pediatric patients undergoing cardiac operations. (J THORAC CardiovasC SuRg 1993;105:712-20)

W. Dietrich, MD, ${ }^{a}$ H. Mössinger, MD, ${ }^{a}$ M. Spannagl, MD, ${ }^{b}$ M. Jochum, MD,${ }^{c}$ P. Wendt, MD, ${ }^{d}$ A. Barankay, MD, ${ }^{\mathrm{a}}$ H. Meisner, MD, ${ }^{\mathrm{e}}$ and J. A. Richter, MD, ${ }^{\mathrm{a}}$ Munich, Germany

From the German Heart Center, Munich; the University Clinic, Munich; and the Technical University, Munich, Germany.

Received for publication March 13, 1992.

Accepted for publication Aug. 4, 1992.

Address for reprints: Wulf Dietrich, MD, German Heart Center Munich, Institute for Anesthesiology, Lothstrasse 11, D-8000 Munich 2, Germany.

${ }^{a}$ Institute for Anesthesiology, German Heart Center, Munich, Germany.

bDepartment of Hematology, University Clinic, Munich, Germany.

'Department of Surgery, Division of Clinical Chemistry, University Clinic, Munich, Germany.
Several recent studies ${ }^{1-8}$ have demonstrated that the application of the protease inhibitor aprotinin during cardiac operations leads to a dramatic reduction of intraoperative and postoperative bleeding tendency. This reduc-

\footnotetext{
${ }^{d}$ Department of Experimental Surgery, Technical University, Munich, Germany.

eDepartment of Cardio-Thoracic Surgery, German Heart Center, Munich, Germany.

Copyright 1993 by Mosby-Year Book, Inc.

$0022-5223 / 93 \$ 1.00+.10 \quad 12 / 1 / 41633$
} 
tion is caused by an attenuation of the hemostatic activation during cardiopulmonary bypass (CPB). Although it is generally accepted that impaired platelet function is the most important factor of postoperative bleeding ${ }^{9}$ and that platelet function is better preserved with aprotinin, ${ }^{10-12}$ the precise mechanism underlying the action of aprotinin is still being discussed: A direct platelet protective effect, ${ }^{12}$ inhibition of fibrinolysis, ${ }^{2,13}$ and inhibition of the contact phase of coagulation ${ }^{4}$ are considered to be the main mechanisms of aprotinin action. However, there is strong evidence supporting the hypothesis that the inhibition of kallikrein and the resulting attenuation of contact phase activation is one important aspect of aprotinin action.

All these studies have been performed on adults having cardiac operations. With respect to coagulation, there are important differences between adults and infants or neonates: The vitamin $\mathrm{K}$-dependent coagulation factors (II, VII, IX, and X) are lower in newborns and attain adult levels between 2 and 12 months of age. ${ }^{14}$ The biologic activity of the contact factors (factor XI, factor XII, prekallikrein, high-molecular-weight kininogen) is depressed to variable degrees. ${ }^{15}$ These differences are more pronounced in immature infants or infants with cyanotic heart disease than in adults. Data were published about the effect of a lower dosage of aprotinin on blood loss in repair of congenital heart defects. ${ }^{16}$ However, no data are available in the literature so far concerning the impact of high-dose aprotinin on the coagulation system in infants and children.

The aim of the present study was to investigate the influence of different aprotinin dosages on the activation of hemostasis during $\mathrm{CPB}$ in pediatric patients having cardiac operations.

\section{Methods}

After institutional approval, 60 pediatric patients with a body weight less than $10 \mathrm{~kg}$, undergoing cardiac operations for different congenital lesions, were enrolled in the protocol. Patients were excluded from the study if the expected duration of $\mathrm{CPB}$ exceeded 120 minutes. Patients were randomly assigned to one of three groups, each consisting of 20 patients. To twenty patients (group L, low-dose protocol) an aprotinin bolus of 15,000 kallikrein-inhibiting units per kilogram ( $\mathrm{KIU} / \mathrm{kg}$ ) (Bayer AG, Leverkusen, Germany) was given after induction of anesthesia and an additional bolus of $15,000 \mathrm{KIU} / \mathrm{kg}$ was added to the pump prime of the heart-lung machine. In the highdose aprotinin group (group H) 20 patients received an aprotinin bolus of 30,000 KIU $/ \mathrm{kg}$ after induction of anesthesia and the same dose was given to the pump prime. The control group (group C) consisted of 20 patients without aprotinin treatment.

Anesthesia. Premedication consisted of morphine $0.2 \mathrm{mg}$ / $\mathrm{kg}$, flunitrazepam $0.04 \mathrm{mg} / \mathrm{kg}$, and atropine $0.01 \mathrm{mg} / \mathrm{kg}$ given 1 hour before induction of anesthesia. For induction, halothane was applied via a face mask ( 0.5 to $1.0 \mathrm{vol} \%)$. Neuromuscular blockade was achieved and maintained with pancuronium $(0.1$ $\mathrm{mg} / \mathrm{kg}$ ). Fentanyl (10 to $20 \mu \mathrm{g} / \mathrm{kg}$ ) and flunitrazepam $(0.02$ $\mathrm{mg} / \mathrm{kg}$ ) were given to deepen and maintain anesthesia. Patients' lungs were ventilated to mild hypocapnia with an air-oxygen mixture or $100 \%$ oxygen. After intubation an arterial catheter (radial artery) and a central venous line were inserted via the right internal jugular vein.

CPB. Mucosa heparin (375 U/kg, La Roche, Basle, Switzerland) was injected via the central venous catheter before aortic cannulation. The extracorporeal circuit consisted of a bubble oxygenator (High Flex D 700 S, Dideco, Mirandola, Italy), nonocclusive roller pumps, and polyvinyl tubing. Blood from the operating field was aspirated by the cardiotomy suction and reinfused to the oxygenator via a $40 \mu$ filter (Dideco D 742). The oxygenator was primed in all patients with $500 \mathrm{ml}$ homologous blood and 100 to $300 \mathrm{ml}$ of crystalloid solution. Heparin $3000 \mathrm{U}$ was added to this homologous blood unit. Two different techniques were applied: (1) Patients operated on with the aid of hypothermia were cooled during bypass to a rectal temperature of $26^{\circ} \mathrm{C}$. The blood flow was kept to $2.4 \mathrm{~L} / \mathrm{min}$ $/ \mathrm{m}^{2}$, being reduced under hypothermia to $1.2 \mathrm{~L} / \mathrm{min} / \mathrm{m}^{2}$. (2) For deep hypothermic circulatory arrest (DHCA), patients were cooled until the rectal temperature was $20^{\circ} \mathrm{C}$. Then perfusion was stopped and the venous cannula was removed from the right atrium. After the surgical procedure, the venous cannula was inserted again and the patients were rewarmed on $C P B$ by means of the heat exchanger of the heart- lung machine and a warming blanket.

$\mathrm{CPB}$ was terminated in all patients when a rectal temperature of $32^{\circ} \mathrm{C}$ was attained. After completion of $\mathrm{CPB}$, residual heparin was neutralized with protamine chloride in a ratio of 1.5 $\mathrm{mg} / 125 \mathrm{U}$ heparin (protamine; La Roche, Basle, Switzerland). One unit of fresh whole blood was available after CPB for all patients from which, depending on hemoglobin value and hemodynamics, different amounts were given. Mechanical ventilation was continued for at least 12 hours after the operation.

Blood samples. Blood samples were taken from the radial artery or, during $\mathrm{CPB}$, from a port of the oxygenator at the following times: (1) after induction of anesthesia before aprotinin infusion, (2) 5 minutes after the onset of CPB, (3) 30 minutes after the onset of CPB or, in case of DHCA 15 minutes after the end of circulatory arrest, (4) at the end of CPB, and (5) at the end of the operation. After the first $5 \mathrm{ml}$ of blood was discarded, blood was drawn into ethylenediaminetetraacetic acid tubes for assessment of hematocrit value, platelet count, and leukocyte count or into acid-citrate-dextrose (ACD) solution (4:1) for all other measurements. The ACD blood was centrifuged at $3000 \mathrm{~g}$ for 10 minutes at room temperature and the plasma was separated from the cellular components. All plasma samples were frozen immediately at $-40^{\circ} \mathrm{C}$ in aliquots and thawed only before testing.

Aprotinin plasma concentrations were quantified by means of a competitive enzyme-linked immunosorbent assay according to Müller-Esterl and associates. ${ }^{17}$ The split products of the crosslinked fibrin were measured by two independent immunoassays, based on monoclonal antibodies to D-dimers (Boehringer, Mannheim, Germany) and to fibrin (Organon Teknika, Heidelberg, Germany). The degradation products of fibrinogen, the total degradation products (Organon Teknika, Heidelberg, Germany), the complex of thrombin with antithrombin III (Behringwerke, Marburg, Germany), F1/F2 prothrombin 
Table I. Demographic data on patients

\begin{tabular}{llccccc}
\hline Group & Age (days) & Weight $(\mathrm{gm})$ & $\begin{array}{c}\text { Operation time } \\
\text { (min) }\end{array}$ & $\begin{array}{c}\text { CPB time } \\
\text { (min) }\end{array}$ & $\begin{array}{c}\text { CCHD } \\
\text { (yes/no) }\end{array}$ & $\begin{array}{c}\text { DHCA } \\
(\text { yes } / \text { no })\end{array}$ \\
\hline Control & $211 \pm 189$ & $5477 \pm 1838$ & $168 \pm 57$ & $84 \pm 31$ & $10 / 10$ & $14 / 6$ \\
Low dose & $263 \pm 189$ & $6178 \pm 1934$ & $200 \pm 47$ & $100 \pm 27$ & $9 / 11$ & $13 / 7$ \\
High dose & $349 \pm 305$ & $6313 \pm 2479$ & $187 \pm 47$ & $98 \pm 40$ & $6 / 14$ & $10 / 10$ \\
\hline
\end{tabular}

$C P B$, Cardiopulmonary bypass; $C C H D$, cyanotic congenital heart disease; $D H C A$, deep hypothermic circulatory arrest.

Table II. Preoperative diagnoses

\begin{tabular}{lcccc}
\hline & Control & Low dose & High dose & Total \\
\hline VSD & 6 & 1 & 8 & 15 \\
TGA* & 5 & 3 & 3 & 11 \\
CAVSD & 1 & 5 & 5 & 11 \\
PA & 3 & 1 & 2 & 6 \\
TOF & 1 & 2 & 1 & 4 \\
SV & 2 & 1 & 0 & 3 \\
TAPVR & 0 & 2 & 0 & 2 \\
TA & 0 & 2 & 0 & 2 \\
TAC & 1 & 1 & 0 & 2 \\
other & 1 & 2 & 1 & 4 \\
Total: & 20 & 20 & 20 & 60 \\
\hline
\end{tabular}

$V S D$, Ventricular septal defect; $T G A$, transposition of the great arteries; $C A V S D$, complete artrioventricular septal defect; $P A$, pulmonary atresia; $T O F$, tetralogy of Fallot; $S V$, single ventricle; $T A P V R$, total anomalous pulmonary venous return; $T A$, tricuspid atresia; $T A C$, truncus arteriosus communis.

*Only atrial level repairs.

fragments (Behringwerke, Marburg, Germany), and elastase in complex with $\alpha_{1}$-protease inhibitor (E. Merck, Darmstadt, Germany) were determined by sandwich enzyme-linked immunosorbent assays using polyclonal and monoclonal antibodies. The concentration of fibrin monomers was measured by an immunoassay using monoclonal antibodies directed against the $N$-terminal $\alpha$-chain of human fibrin (Boehringer, Mannheim, Germany).

Spontaneous fibrinolytic activation in the native samples and in their euglobulin fraction was estimated by the use of plasminogen containing human fibrin plates. ${ }^{18}$ Any development of a lysis area, regardless of its size, was considered to be an indication of extrinsic plasminogen activator(s) in the sample. The activated clotting time was determined according to the instructions of the manufacturer (Hemochron 400, International Technidyne Corp., Edison, N.J.). For global coagulation tests, routinely applied clotting methods were used. Blood loss through the chest tubes was measured in the intensive care unit 6,12 , and 24 hours after the operation. Because body weight varied substantially among patients, the blood loss was expressed as a function of the body surface area in milliliters per square meter.

Data analysis. Two-way analysis of variance was used to analyze normal distributed data. Whenever appropriate, significant differences among the three groups were explored with the Newman-Keuls test. Parametric data were given as mean \pm standard deviation. If Shapiro's test of normality revealed that data did not conform to a normal distribution, comparison among the three groups was done with the Kruskal-Wallis test.
The $\chi^{2}$ test was used for categoric data. Stepwise multiple regression analysis was performed to assess the independent contributions of group allocation, cyanotic heart disease, or the application of DHCA to changes of hemostatic variables at the end of CPB. Linear regression analysis was applied to examine the relationship between the temperature 30 minutes after onset of CPB and the degree of fibrin formation and the concentration of the total degredation products. A $p$ value less than 0.05 was considered statistically significant.

\section{Results}

All 60 patients admitted to the study were included to the subsequent analysis. Table I shows the demographic data for the three groups and Table II the preoperative diagnoses. No significant differences were found for any of the variables. The mean CPB times including circulatory arrest times were $84 \pm 31$ minutes (group C), $100 \pm 27$ minutes (group L), and $98 \pm 40$ minutes (group H), respectively. Fourteen (group C), 13 (group $\mathrm{L}$ ), and 10 patients (group $\mathrm{H}$ ) were operated on in DHCA. The circulatory arrest time was $34 \pm 17$ minutes in group $\mathrm{C}(n=14)$ compared with $48 \pm 17$ minutes in group $\mathrm{L}(n=13)$ and $55 \pm 12$ minutes in group $\mathrm{H}$ $(n=10)(p<0.05$, group $\mathrm{C}$ versus groups $\mathrm{L}$ and $\mathrm{H})$. The mean CPB time, not including the arrest time for all infants with DHCA $(n=37)$, was $56 \pm 25$ minutes, whereas the $\mathrm{CPB}$ time was $81 \pm 38$ minutes in patients without DHCA $(n=23)(p<0.05)$.

The highest aprotinin plasma concentrations were measured 30 minutes after the onset of CPB: $73 \pm 30$ $\mathrm{KIU} / \mathrm{ml}$ in group $\mathrm{L}$ and $99 \pm 25 \mathrm{KIU} / \mathrm{ml}$ in group $\mathrm{H}$. At the end of CPB the concentrations were $63 \pm 72 \mathrm{KIU} /$ $\mathrm{ml}$ (group L) and $92 \pm 20 \mathrm{KIU} / \mathrm{ml}$ (group H), respectively. Patients of group $\mathrm{L}$ received a total amount of $98,000 \pm 42,000 \mathrm{KIU}$ aprotinin, as compared with $180,000 \pm 81,000 \mathrm{KIU}$ in group $\mathrm{H}(p<0.05)$.

The concentration of the $\mathrm{F} 1 / \mathrm{F} 2$ prothrombin fragments and the thrombin-antithrombin III complex increased steadily during $\mathrm{CPB}$. At the end of the operation the $\mathrm{F} 1 / \mathrm{F} 2$ concentrations were $9.9 \pm 4.9 \mathrm{ng} / \mathrm{ml}$ (group C), $11.2 \pm 4.8 \mathrm{ng} / \mathrm{ml}$ (group L), and $6.3 \pm 6.0$ $\mathrm{ng} / \mathrm{ml}$ (group $\mathrm{H})(p<0.05$ versus groups $\mathrm{C}$ and $\mathrm{L}$ ). In contrast, the thrombin-antithrombin III complex did not show significant differences. The course of the concentra- 


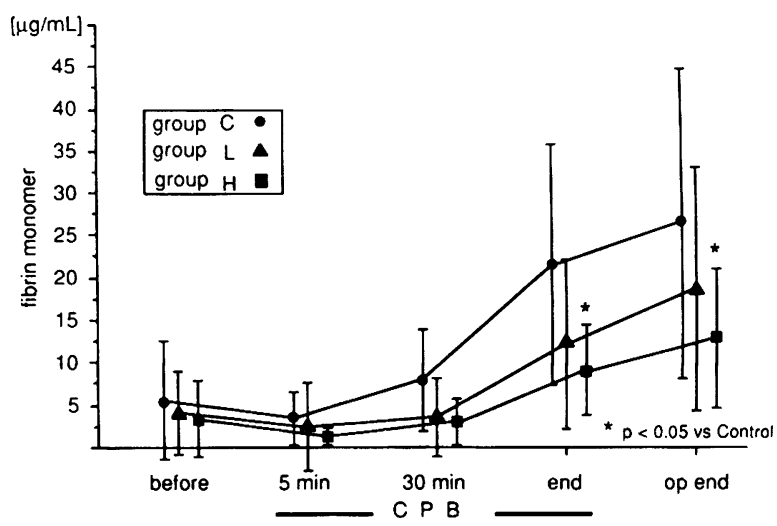

Fig. 1. Fibrin monomer concentration increased during $C P B$ in all three groups. However, the increase in the high-dose aprotinin group (group $\mathrm{H}$ ) was significantly attenuated by aprotinin. This indicates reduced clotting activation with aprotinin treatment.

tion of fibrin monomers is given in Fig. 1. The level was significantly higher in the control group $(21.6 \pm 14$ $\mu \mathrm{g} / \mathrm{ml})$ than in groups $\mathrm{L}$ and $\mathrm{H}(12.2 \pm 10$ and $9.2 \pm 5.2$ $\mu \mathrm{g} / \mathrm{ml}$, respectively) ( $p<0.05$ versus group C). There was a significant correlation between the formation of fibrin monomers and the concentration of aprotinin in the plasma at the end of CPB (Fig. 2).

The course of the concentration of fibrin-fibrinogen split products is given in Fig. 3. Aprotinin showed a dosedependent effect on the development of split products. At the end of CPB the concentrations of fibrin-fibrinogen split products were $6.7 \pm 3.5 \mu \mathrm{g} / \mathrm{ml}$ in group $\mathrm{C}$, $3.4 \pm 3.0 \mu \mathrm{g} / \mathrm{ml}$ in group $\mathrm{L}$, and $1.5 \pm 0.6 \mu \mathrm{g} / \mathrm{ml}$ in group $\mathrm{H}(p<0.05)$. The course of the concentration of D-dimers was nearly identical (D-dimer concentrations at the end of the operation: $1.2 \pm 0.8 \mu \mathrm{g} / \mathrm{ml}$, group $\mathrm{C}$; $0.9 \pm 0.9 \mu \mathrm{g} / \mathrm{ml}$, group $\mathrm{L}$; and $0.5 \pm 0.4 \mu \mathrm{g} / \mathrm{ml}$, group H) $(p<0.05$ versus group C). The fibrin split products paralleled the course of the D-dimers (Fig. 4). Thirty minutes after the onset of $\mathrm{CPB}$, the fibrin plates showed positive results in $75 \%$ of patients in group C, in $65 \%$ in group $\mathrm{L}$, and in $35 \%$ in group $\mathrm{H}$ (the lysis areas were $15.8 \pm 20.1 \mathrm{~mm}^{2}, 8.7 \pm 8.5 \mathrm{~mm}^{2}$, and $1.7 \pm 3.8 \mathrm{~mm}^{2}$ in groups $\mathrm{C}, \mathrm{L}$, and $\mathrm{H}$, respectively; $p<0.05$ ). There were no differences in the results on the fibrin plates at the end of $\mathrm{CPB}$ or the end of the operation.

No significant correlation could be found between rectal temperatures measured 30 minutes after onset of $\mathrm{CPB}$ and the formation of fibrin or fibrin degradation products. However, there was a tendency in all three groups toward a higher degree of activation of coagulation and fibrinolysis with lower body temperatures. Multivariate analysis

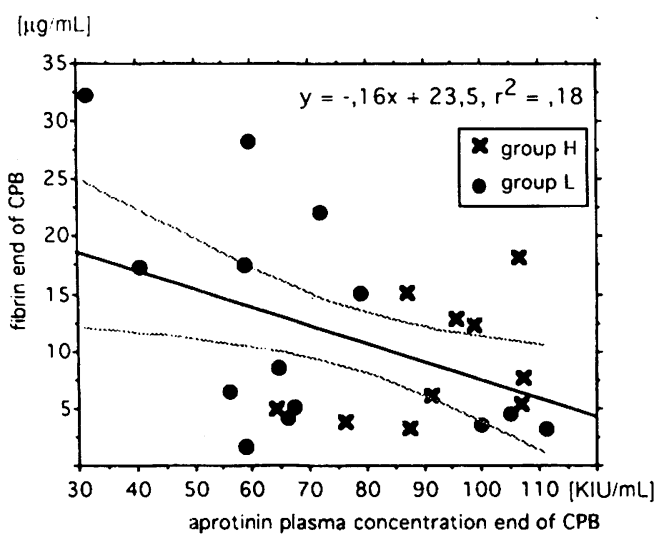

Fig. 2. Correlation of aprotinin plasma concentration at the end of CPB and fibrin monomer concentration. The higher the aprotinin plasma concentration the lower the fibrin generation.

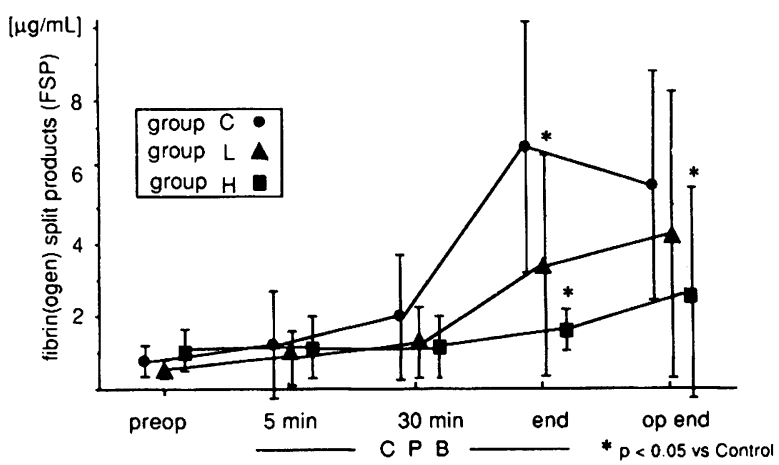

Fig. 3. The total split products of fibrinogen and fibrin (FSP) demonstrated a dose-dependent reduction during CPB with aprotinin treatment. This indicates less fibrinolytic activity with aprotinin treatment.

identified only the group allocation as being independently associated with a decreased clotting activation at the end of extracorporeal circulation. However, regardless of group allocation, all patients operated on in DHCA showed at the end of $\mathrm{CPB}$ increased concentrations of fibrin-fibrinogen split products (with DHCA, $4.6 \pm 3.8$ $\mu \mathrm{g} / \mathrm{ml}$; without, $2.7 \pm 2.2 \mu \mathrm{g} / \mathrm{ml} ; p=0.05$ ) and fibrin split products (with DHCA, $2.7 \pm 2.9 \mu \mathrm{g} / \mathrm{ml}$; without, $1.2 \pm 1.4 \mu \mathrm{g} / \mathrm{ml} ; p<0.05$ ) compared with patients without DHCA. On the other hand, there were no differences in regard to hemostatic activation between cyanotic and acyanotic patients.

A continuous increase of the concentration of the complex bound elastase over $\mathrm{CPB}$ time was noted. At the end of CPB these concentrations were $784 \pm 278 \mathrm{ng} / \mathrm{ml}$ (group C), $693 \pm 189 \mathrm{ng} / \mathrm{ml}$ (group L), and $719 \pm 270$ 

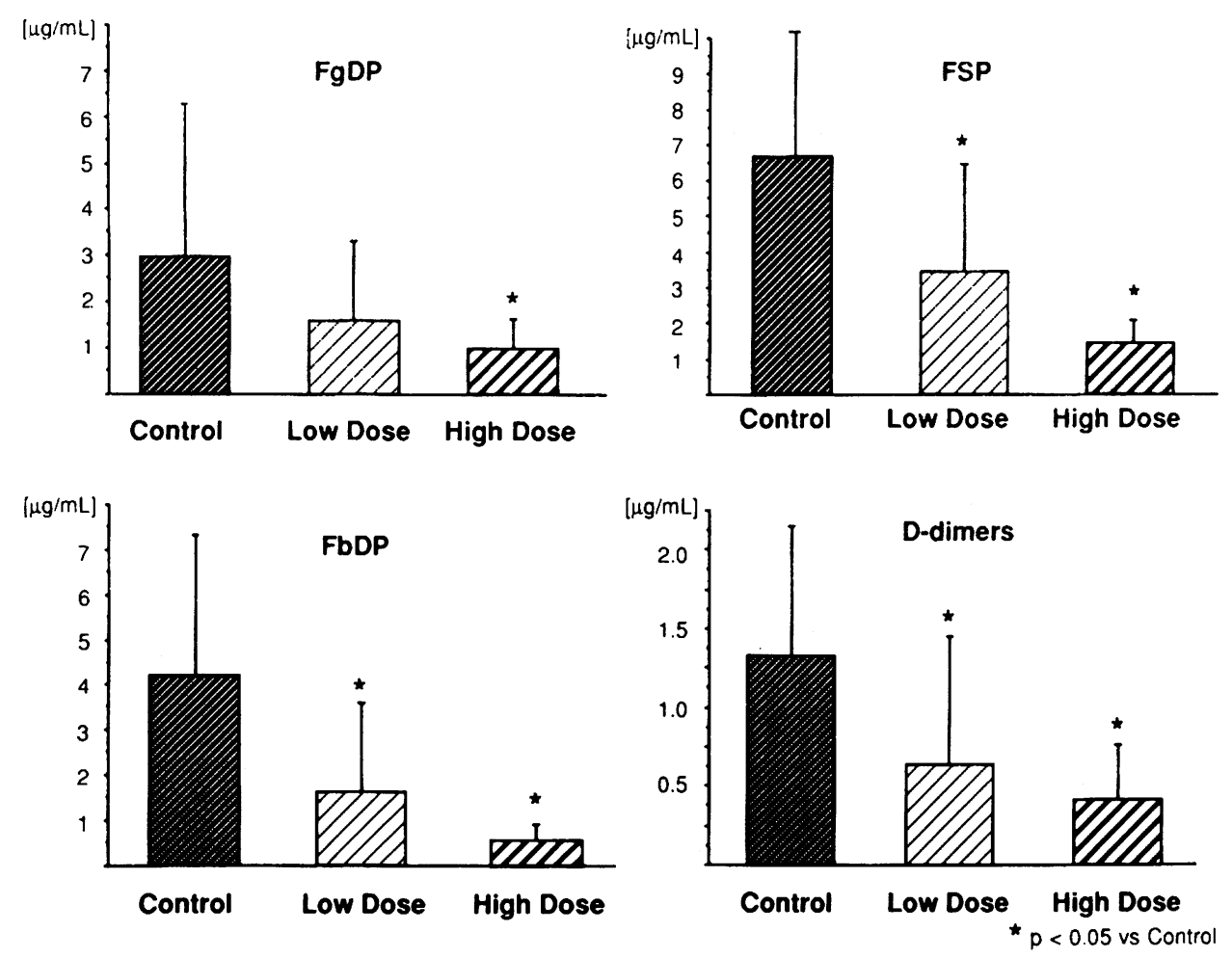

Fig. 4. Concentrations of different split products of fibrinogen or fibrin at the end of CPB. D-dimers and split products of fibrin $(F b D P)$ were measured by different immunoassays. Total degradation products (FSP) are the split products of fibrin and fibrinogen, whereas fibrinogen degradation products $(F g D P)$ were determined by monoclonal antibodies against fibrinogen split products. There was a dose-dependent reduction of all split products at the end of $\mathrm{CPB}$, indicating reduced fibrinolytic activity with aprotinin treatment.

$\mathrm{ng} / \mathrm{ml}$ (group H) ( $\left.p=\mathrm{NS}^{*}\right)$. The platelet count did not reveal significant differences among the three groups. From normal preoperative values the counts dropped to $121 \pm 36 \times 10^{9} / \mathrm{L}\left(\right.$ group C), $93 \pm 37 \times 10^{9} / \mathrm{L}$ (group $\mathrm{L}$ ), and $102 \pm 44 \times 10^{9} / \mathrm{L}$ (group H) at the end of the operation $(p=\mathrm{NS})$. The preoperative hemoglobin concentration varied from $13.0 \pm 2.3 \mathrm{gm} / \mathrm{dl}$ (group C) to $14.1 \pm 3.4 \mathrm{gm} / \mathrm{dl}$ (group L) and $12.8 \pm 2.1 \mathrm{gm} / \mathrm{dl}$ (group L) $(p=\mathrm{NS})$. At the end of CPB hemoglobin values were $10.6 \pm 1.4 \mathrm{gm} / \mathrm{dl}$ (group C), $10.9 \pm 2.0 \mathrm{gm} / \mathrm{dl}$ (group L), and $10.9 \pm 1.3 \mathrm{gm} / \mathrm{dl}$ (group $\mathrm{H}$ ), respectively.

Blood losses 6 hours postoperatively were $164 \pm 87$ $\mathrm{ml} / \mathrm{m}^{2}$ (group C), $160 \pm 106 \mathrm{ml} / \mathrm{m}^{2}$ (group L), and $99 \pm 32 \mathrm{ml} / \mathrm{m}^{2}$ (group $\left.\mathrm{H}\right)(p<0.05$, group $\mathrm{H}$ versus group $\mathrm{C}$ or $\mathrm{L}$ ). However, there were no significant differences in 24-hour blood losses: $294 \pm 148 \mathrm{ml} / \mathrm{m}^{2}$ (group C), $278 \pm 162 \mathrm{ml} / \mathrm{m}^{2}$ (group L), and $199 \pm 67 \mathrm{ml} / \mathrm{m}^{2}$ (group H) (range, 78 to $603 \mathrm{ml} / \mathrm{m}^{2}$, group C; 79 to 761

*NS = Not significant. $\mathrm{ml} / \mathrm{m}^{2}$, group L; and 103 to $370 \mathrm{ml} / \mathrm{m}^{2}$, group $\mathrm{H}$ ). There were no differences in the required units of homologous blood among the groups. Two units of fresh whole blood were available for all patients. The oxygenator of the heart-lung machine was primed with the first unit, and different amounts from the second unit were given during and after the operation. In none of the patients was a third unit of bank blood necessary.

The activated clotting time was greater than $1000 \mathrm{sec}-$ onds in all groups during CPB. None of the patients needed additional heparin to keep the activated clotting time above 400 seconds. After heparin reversal by protamine chloride, the activated clotting times were $139 \pm 19$ seconds (group C), $135 \pm 23$ seconds (group L), and $136 \pm 23$ seconds (group H) $(p=$ NS). The times to control bleeding, that is, the intervals between end of CPB and thoracic closure, were between $60 \pm 17$ minutes (group H) and $63 \pm 14$ minutes (group C) $(p=\mathrm{NS})$. No side effects attributable to aprotinin were observed. All patients survived the early postoperative period. 


\section{Discussion}

This investigation corroborates the results of recent studies ${ }^{2}, 6,19,20$ on the use of high-dose aprotinin in adults having cardiac operations. In the present study we found a dose-dependent attenuation of the deleterious effect of $\mathrm{CPB}$ on hemostatic activation, as well as a reduction of bleeding tendency, in our group of children undergoing cardiac operations with aprotinin compared with the control group without aprotinin treatment. Duration of operation and $\mathrm{CPB}$, as well as preoperative hemoglobin concentration, were comparable among the groups, thus rendering negligible the influence of different surgical procedures on hemostatic alterations.

Several studies have shown that clotting activation and fibrinolysis takes place during CPB. ${ }^{21,22}$ This process starts with the activation of factor XII (Hageman factor) by the initial blood contact with the unphysiologic, nonendothelial surfaces of the circuit of the heart-lung machine. The contact activation with the conversion of prekallikrein to kallikrein launches the activation of the cascades systems of the body, including the intrinsic coagulation pathway, fibrinolysis, the classic complement pathway, and the kinin-kininogen system. Contact activation does not play a role in physiologic coagulation. The extracorporeal circuit, however, with its artificial surfaces, is a highly unphysiologic system. Therefore, under the condition of $\mathrm{CPB}$ contact activation contributes to the activation of hemostasis. Neutrophil activation takes place at the same time. Heparin treatment is supposed to inhibit only one component of this contact activation system, the formation of fibrin. Because thrombin already bound to fibrin is less inhibitable by heparin, ${ }^{23}$ fibrin formation and polymerization take place despite heparin treatment during $\mathrm{CPB} .{ }^{24}$

Platelets are activated either by contact with unphysiologic surfaces ${ }^{25}$ by generated thrombin ${ }^{26,27}$ or plasmin, ${ }^{28}$ which are both powerful platelet stimulators. ${ }^{29}$ It is known that impaired platelet function is the main cause of bleeding after cardiac operations. ${ }^{30}$ Thus the link between hemostatic and platelet activation is as follows: Less plasmin and thrombin formation causes less platelet activation. This assumption is corroborated by the results of several studies demonstrating better preservation of platelet function in patients with high-dose aprotinin treatment. ${ }^{10-12}$

Our study clearly proved a dose-dependent reduction in fibrinolytic activity with aprotinin: Patients in the highdose group had the lowest concentrations of fibrin-fibrinogen split products, control patients the highest, and patients in the low-dose group had intermediate concentrations. The results of the fibrin plates also demonstrat- ed less fibrinolytic activity. However, the given dosages of aprotinin were not able to suppress fibrinolytic activity on fibrin plates completely. Besides this antifibrinolytic effect, a less pronounced antithrombin effect was also evident: We found significantly less fibrin formation in the patient group with the highest aprotinin dosage, and the concentrations of the F1/F2 fragments were significantly reduced at the end of the operation. However, the thrombin-antithrombin III-complex concentrations were not different among the three groups. With respect to plasma levels of complexed elastase, we did not see any differences in neutrophil activation, which indicates that clotting and fibrinolysis products play a minor role in neutrophil stimulation.

Several studies have shown the antifibrinolytic effect of aprotinin treatment during $\mathrm{CPB} .{ }^{2,13}$ Aprotinin is supposed to manifest its antifibrinolytic properties in plasma concentrations of about $50 \mathrm{KIU} / \mathrm{ml},{ }^{31,32}$ whereas kallikrein inhibition is achieved only with higher concentrations. ${ }^{33}$ It seems to be of paramount importance that aprotinin not only acts as an antifibrinolytic agent but also inhibits thrombin generation, ${ }^{4,11}$ thus enhancing the anticoagulatory effect of heparin during $\mathrm{CPB}{ }^{34}$ This is the pivotal difference to therapy with solely antifibrinolytic agents. ${ }^{35}$

The present data show some differences compared with results from adult patients treated with aprotinin. Recently, a reduction of the thrombin-antithrombin III-complex $^{4,11}$ and fibrin formation ${ }^{36}$ in adult patients undergoing myocardial revascularization could be demonstrated. The difference in these findings may be based on the different aprotinin plasma concentrations: The peak concentration found in adult patients was around $300 \mathrm{KIU} /$ $\mathrm{ml},{ }^{4}$ whereas in our study the peak aprotinin plasma concentration in the high-dose group was $99 \pm 25 \mathrm{KIU} /$ $\mathrm{ml} 30$ minutes after the onset of CPB. According to the dosage introduced by Royston, ${ }^{7}$ Bidstrup, ${ }^{37}$ and their associates, adult patients received 5 to $6 \times 10^{6} \mathrm{KIU}$ aprotinin during a cardiac operation. This represents approximately $60,000 \mathrm{KIU} / \mathrm{kg}$ body weight. Thus we calculated the dosage in the high-dose group according to this regimen with $2 \times 30,000 \mathrm{KIU} / \mathrm{kg}$. However, the relation between circulating blood volume and the pump prime differs when these small patients are compared with adults. Therefore the diluting effect of the pump prime is more pronounced, resulting in lower plasma concentrations of aprotinin during CPB. Consequently, the priming volume of the heart-lung machine should be included in the calculation of the aprotinin dosage. One might anticipate even more pronounced effects on hemostasis at aprotinin doses closer to those achieved in adults. 
Another difference between infants and adults was the prolonged activated clotting time in our pediatric patients. Because of the immature hemostatic system, the prothrombin time and the partial thromboplastin time $^{14,15,38}$ in neonates and infants are prolonged. Furthermore, hepatic congestion with an impaired development of clotting factors and polycythemia with imbalance of cellular and plasmatic hemostatic components is common in congenital heart disease.$^{39}$ However, a more simple explanation for this difference may be based on the fact that our patients received $3000 \mathrm{U}$ of additional heparin per unit of homologous blood during CPB. Therefore these patients were heparinized more effectively, because the total dosage of heparin per kilogram was higher than in adult patients.

A secondary result of this study was that the temperature reduction did not result in reduced hemostatic activation. In contrast, patients operated on under DHCA showed a tendency toward higher activation of the hemostatic system. Thus hypothermia does not seem to protect from activation of the hemostatic system.

The 6-hour postoperative blood loss was significantly lower in the high-dose aprotinin group than in the control group. Because postoperative blood loss is not the main predictor of transfusion requirement in patients with congenital heart disease, the homologous blood requirement was not significantly different among the groups. Moreover, in our institution homologous blood is available only in units containing 450 to $500 \mathrm{ml}$ blood. Regardless of their group allocation, all patients received different amounts from 2 units of homologous blood during the operation and in the early postoperative period and therefore came into contact with the blood of two blood donors. However, because patients with an expected bypass time longer than 2 hours were excluded from the study (e.g., anatomic repair of transposition of the great arteries), we presumably deal with a bias toward lower intraoperative and postoperative blood loss. This exclusion was done for ethical reasons, because we did not want to withhold aprotinin in these longer operations in which the blood-saving effect of this drug was clinically obvious before this study. In our experience, after introduction of routine aprotinin treatment, a dry operative field is the rule, whereas during the time without aprotinin, surgical hemostasis was often a long-lasting endeavor.

What are the true merits of aprotinin treatment? Clearly, for adult patients with cardiac disease to date, aprotinin reduces bleeding tendency caused by $\mathrm{CPB}^{40,41}$ and thereby reduces homologous blood requirement. This reduction in bleeding is the consequence of better preservation of the hemostatic system. The present study dem- onstrated a statistically significant reduction of bleeding tendency also for pediatric patients. However, in terms of transfusion requirements it was clinically insignificant. This might be due to patient selection (patients with expected long bypass times were excluded) or to the setup of the heart-lung machine used in our institution (routine administration of 1 unit of blood to the pump prime. On the other hand, this study showed a significant reduction of clotting and fibrinolytic activation. Because contact activation during CPB leads to stimulation of other cascade systems, ${ }^{42,43}$ the attenuation of hemostasis found in this study is a favorable effect of aprotinin treatment.

The positive effect of aprotinin could be superseded by possible side effects. As a foreign protein, aprotinin has antigenic properties resulting in antibody formation. Whether this formation might cause severe sequelae during reexposure to aprotinin, which has to be anticipated in operations for congenital heart disease, has not yet been settled. Further studies will be necessary to clarify this issue.

In summary, the present data suggest that high-dose aprotinin treatment attenuates hemostatic activation during $\mathrm{CPB}$ in pediatric patients. The maintenance of a hemostatic equilibrium postoperatively caused by diminished clotting and fibrinolytic activity is the main effect of aprotinin treatment. The higher dosage of $2 \times 30,000$ $\mathrm{KIU} / \mathrm{kg}$ was more effective than the dosage of $2 \times 15,000$ $\mathrm{KIU} / \mathrm{kg}$. Comparing these results with data gained in adults, one might postulate that an even higher aprotinin dosage would be desirable. Although it was not possible to demonstrate an overall saving of homologous blood in these small patients, the reduction of hemostatic activation and bleeding tendency is valuable. Therefore we recommend the routine use of aprotinin in pediatric patients having cardiac operations.

\section{REFER E N C ES}

1. Alajmo F, Calamai G, Perna AM, et al. High-dose aprotinin: hemostatic effects in open-heart operations. Ann Thorac Surg 1989;48:536-9.

2. Blauhut B, Gross C, Necek S, Doran JE, Spath P, Lundsgaardhansen $P$. Effects of high-dose aprotinin on blood loss, platelet function, fibrinolysis, complement, and renal function after cardiopulmonary bypass. J THORAC CARDIOVASC SURG 1991;101:958-67.

3. Dietrich W, Barankay A, Dilthey G, et al. Reduction of homologous blood requirement in cardiac surgery by intraoperative aprotinin application: clinical experience in 152 cardiac surgical patients. Thorac Cardiovasc Surg 1989; 37:92-8.

4. Dietrich W, Spannagl M, Jochum M, et al. Influence of 
high-dose aprotinin treatment on blood loss and coagulation pattern in patients undergoing myocardial revascularization. Anesthesiology 1990;73:1119-26.

5. Fraedrich G, Weber C, Bernard C, Hettwer A, Schlosser V. Reduction of blood transfusion requirement in open heart surgery by administration of high doses of aprotinin: preliminary results. Thorac Cardiovasc Surg 1989;37:8991.

6. Havel M, Teufelsbauer H, Knobl P, et al. Effect of intraoperative aprotinin administration on postoperative bleeding in patients undergoing cardiopulmonary bypass operation. J Thorac Cardiovasc Surg 1991;101:968-72.

7. Royston D, Taylor KM, Bidstrup BP, Sapsford RN. Effect of aprotinin on need for blood transfusion after repeat open-heart surgery. Lancet 1987;2:1289-91.

8. van Oeveren W, Jansen NJG, Bidstrup BP, et al. Effects of aprotinin on hemostatic mechanisms during cardiopulmonary bypass. Ann Thorac Surg 1987;44:640-5.

9. Harker LA. Bleeding after cardiopulmonary bypass. $\mathrm{N}$ Engl J Med 1986;314:446-8.

10. Lavee J, Savion N, Smolinsky A, Goor D, Mohr R. Platelet protection by aprotinin in cardiopulmonary bypass: electron microscopic study. Ann Thorac Surg 1992;53:47781 .

11. Lu H, Soria C, Commin PL, et al. Hemostasis in patients undergoing extracorporeal circulation: the effect of aprotinin (Trasylol). Thromb Haemost 1991;66:633-7.

12. van Oeveren W, Harder MP, Roozendaal KJ, Eijsman L, Wildevuur CRH. Aprotinin protects platelets against the initial effect of cardiopulmonary bypass. J THORAC CARDIOVASC SURG 1990;99:788-97.

13. Marx G, Pokar H, Doering V, Tilsner V. The effects of aprotinin on hemostatic function during cardiac surgery. $\mathrm{J}$ Cardiothorac Vasc Anesth 1991;5:467-74.

14. Ming Y, Simon N, Maertens P, Brigham S, Liu P. Maternal-fetal transport of vitamin $\mathrm{K} 1$ and its effect on coagulation in premature infants. J Pediatr 1989;115:1009-13.

15. Andrew M, Bhogal M, Karpatkin M. Factors XI and XII and prekallekrein in sick and healthy premature infants. $\mathrm{N}$ Engl J Med 1981;305:1130-3.

16. Popov-Cenic S, Urban AE, Noë G. Studies on the cause of bleeding during and after surgery with a heart-lung machine in children with cyanotic and acyanotic congenital cardiac defects and their prophylactic treatment. In: The role of chemical mediators in the pathophysiology of acute illness and injury. New York: McConn, 1982:229-42.

17. Müller-Esterl W, Oettl A, Truscheit E, Fritz H. Monitoring of aprotinin plasma levels by an enzyme-linked immunosorbent assay (ELISA). Fresenius Z Anal Chem 1984; 317:718-22.

18. Wendt P, Fritsch A, Schulz F, Wunderlich G, Blümel G. Proteinases and inhibitors in plasma and peritoneal exudate in acute pancreatitis. Hepatogastroenterology 1984;31: 277-81.

19. Bidstrup BP, Royston D, Sapsfort RN, Taylor KM. Reduction in blood loss and blood use after cardiopulmo- nary bypass with high-dose aprotinin (Trasylol). J THORAC CARdiOVASC SURG 1989;97:364-72.

20. Harder MP, Eijsman L, Roozendaal KJ, van Oeveren W, Wildevuur CRH. Aprotinin reduces intraoperative and postoperative blood loss in membrane oxygenator cardiopulmonary bypass. Ann Thorac Surg 1991;51:936-41.

21. Kirklin JK, Westaby S, Blackstone EH, Kirklin JW, Chenoweth DE, Pacifico AD. Complement and the damaging effects of cardiopulmonary bypass. J THORAC CARDIOVASC SURG 1983;86:845-57.

22. van Oeveren W, Kazatchkine MD, Descamps-Latscha B, et al. Deleterious effects of cardiopulmonary bypass: a prospective study of bubble versus membrane oxygenation. $\mathrm{J}$ ThoraC CARDIOVASC SURG 1985;89:888-9.

23. Weitz J, Hudoba M, Massel D, Maraganore J, Hirsch J. Clot-bound thrombin is protected from inhibition by heparin-antithrombin III but is susceptible to inactivation by antithrombin III-independent inhibitors. J Clin Invest 1990;86:385-91.

24. Dietrich W, Spannagl M, Schramm W, Vogt W, Barankay A, Richter J. The influence of preoperative anticoagulation on heparin response during cardiopulmonary bypass. J Thorac Cardiovasc SuRG 1991;102:505-14.

25. Zilla P, Fasol R, Groscurth P, Klepetko W, Reichenspurner $\mathrm{H}$, Wolner E. Blood platelets in cardiopulmonary bypass operations: Recovery occurs after initial stimulation, rather than continual activation. J THORAC CARDIOVASC SURG 1989;97:379-88.

26. Hanson S, Harker L. Interruption of acute platelet-dependent thrombosis by the synthetic antithrombin D-phenylalanyl-L-prolyl-arginyl chloromethylketone. Proc Natl Acad Sci USA 1988;85:3184-8.

27. Winters K, Santoro S, Miletich J, Eisenberg P. Relative importance of thrombin compared with plasmin-mediated platelet activation in response to plasminogen activation with streptokinase. Circulation 1991;84:1552-60.

28. Lu H, Soria C, Cramer EM, et al. Temperature dependence of plasmin-induced activation or inhibition of human platelets. Blood 1991;77:996-1005.

29. Edmunds LH. Blood platelets and bypass [Letter]. J THORAC Cardiovasc Surg 1989;97:470-1.

30. Mammen EB, Koets MH, Washington BC. Hemostasis changes during cardiopulmonary bypass surgery. Semin Thromb Hemost 1985; 11:281-92.

31. Emerson FE. Pharmacology of aprotinin and efficacy during cardiopulmonary bypass. Cardiovasc Drug Rev 1989; 7:127-40.

32. Verstraete M. Clinical application of inhibitors of fibrinolysis. Drugs 1985;29:236-61.

33. Fritz H, Wunderer G. Biochemistry and applications of aprotinin, the kallikrein inhibitor from bovine organs. Arzneim Forsch/Drug Res 1983;33:479-94.

34. DeSmet AAEA, Joen MCN, van Oeveren W, et al. Increased anticoagulation during cardiopulmonary bypass by aprotinin. J THORAC CARDIOVASC SURG 1990;100: 520-7. 
35. Horrow JC, Hlavacek J, Strong MD, et al. Prophylactic tranexamic acid decreases bleeding after cardiac operations. J Thorac Cardiovasc Surg 1990;99:70-4.

36. Dietrich W, Richter JA, Schramm W, Spannagl M. What is the mechanism of action of aprotinin? [Reply.] Anesthesiology 1991;75:378-9.

37. Bidstrup BP, Royston D, Sapsford RN, Taylor KM. Effect of aprotinin on need for blood transfusion in patients with septic endocarditis having open heart surgery. Lancet 1988; 1:366-7.

38. Stuart M. Bleeding in newborn and pediatric patients. In: Colman R, Hirsh J, Marder V, Salzman E, eds. Hemostasis and thrombosis. Philadelphia: JB Lippincott, 1987:94259.

39. Henriksson P, Värendh G, Lundström N. Hemostatic de- fects in cyanotic congenital heart disease. Br Heart $\mathbf{J}$ 1980;41:23.

40. Royston D. High-dose aprotinin therapy: a review of the first five years' experience. J Cardiothorac Vasc Anesth 1992;6:76-100.

41. Dietrich W, Barankay A, Hähnel C, Richter J. High-dose aprotinin in cardiac surgery: three years' experience in 1,784 patients. J Cardiothorac Vasc Anesth 1992;6:324-7.

42. Chenoweth DE, Cooper SW, Hugli TE, Stewart RW, Blackstone EH, Kirklin J. Complement activation during cardiopulmonary bypass: evidence for generation of $\mathrm{C} 3 \mathrm{a}$ and C5a anaphylatoxins. N Engl J Med 1981;304:497-503.

43. Dreyer WJ, Smith CW, Entman ML. Invited letter concerning: Neutrophil activation during cardiopulmonary bypass. J ThOrac Cardiovasc Surg 1991;102:318-26.

\section{Bound volumes available to subscribers}

Bound volumes of THE JOURNAL OF THORACIC AND CARDIOVASCULAR SURGERY are available to subscribers (only) for the 1993 issues from the Publisher, at a cost of $\$ 75.00$ for domestic, $\$ 98.25$ for Canadian, and $\$ 93.00$ for international subscribers for Vol. 105 (JanuaryJune) and Vol. 106 (July-December). Shipping charges are included. Each bound volume contains a subject and author index and all advertising is removed. Copies are shipped within 60 days after publication of the last issue of the volume. The binding is durable buckram with the JourNAL name, volume number, and year stamped in gold on the spine. Payment must accompany all orders. Contact Mosby, Subscription Services, 11830 Westline Industrial Drive, St. Louis, Missouri 63146-3318, USA; phone (800) 325-4177, ext. 4351, or (314) 453-4351.

Subscriptions must be in force to qualify. Bound volumes are not available in place of a regular JourNal subscription. 


\title{
The Journal of THORACIC AND
}

\section{CARDIOVASCULAR SURGERY}

\author{
Official organ, The American Association for Thoracic Surgery \\ and The Western Thoracic Surgical Association
}

\author{
Editor JOHN W. KIRKLIN, Birmingham, Ala. \\ Associate editor EUGENE H. BLACKSTONE, Birmingham, Ala. \\ Advisory editorial board CARY W. AKINS, Boston \\ LEONARD L. BAILEY, Loma Linda, Calif. \\ JOHN C. BALDWIN, New Haven, Conn. \\ ROBERT H. BARTLETT, Ann Arbor \\ WILLIAM A. BAUMGARTNER, Baltimore \\ LAWRENCE I. BONCHEK, Lancaster, Pa. \\ EDWARD L. BOVE, Ann Arbor \\ GERALD D. BUCKBERG, Los Angeles \\ ALDO R. CASTANEDA, Boston \\ PAT O. DAILY, San Diego \\ TOM R. DEMEESTER, Los Angeles \\ WILLIAM JEFFERY DREYER, Houston \\ L. HENRY EDMUNDS, JR., Philadelphia \\ L. PENFIELD FABER, Chicago \\ MICHEL N. ILBAWI, Oak Lawn, III. \\ SHUKRI F. KHURI, Boston \\ GERALD M. LAWRIE, Houston \\ BRUCE W. LYTLE, Cleveland \\ JAMES B. D. MARK, Stanford \\ LYNDA L. MICKLEBOROUGH, Toronto \\ G. A. PATTERSON, St. Louis \\ ERIC A. ROSE, New York \\ VALERIE RUSCH, New York \\ NORMAN A. SILVERMAN, Detroit \\ HENRY M. SPOTNITZ, New York \\ ANDREW S. WECHSLER, Richmond, $v a$. \\ RICHARD D. WEISEL, Toronto \\ Editorial coordinator \\ Senior manuscript editor \\ Former Editors \\ ANN EARWOOD, Birmingham, Ala. \\ LOIS R. LINDLEY, St. Louis \\ EVARTS A. GRAHAM, Editor, 1931-1957 \\ EMILE HOLMAN， Editor. 1957-1962 \\ BRIAN BLADES, Editor, 1962-1977 \\ DWIGHT C. McGOON, Editor, 1977-1987 \\ Volume 105, January-June, 1993 \\ Mosby \\ St. Louis, Mo.
}




\section{Volume 105}

Copyright $\odot 1993$ by Mosby-Year Book, Inc.

All rights reserved

Printed in the United States of America

\section{N/ Mosby}




\section{Index to volume 105}

\section{Author index*}

A

Abd-Elfattah AS, Messier RH Jr, Domkowski PW, Jones JL, Aly HM, Crescenzo DG, Wallace RB, Hopkins RA. Inhibition of adenosine deaminase and nucleoside transport: utility in a model of homograft cardiac valve preimplantation processing. 1993;105:1095-105

Abdelnoor M (see Øvrum et al). 1993;105:78-83

Acar C (see Allen et al). 1993; 105:864-84

Acuff T (see Hazelrigg et al). 1993; 105:389-93

Adoumie R, Shennib H, Brown R, Slinger P, Chiu RC-J. Differential lung ventilation: applications beyond the operating room. 1993; 105:229-33

Aeba R (see Hardesty et al). 1993;105:660-6

Aebert H, Laas J, Borst HG. Thoracic wall necrosis in a patient with intemal mammary-coronary bypass after prosthetic replacement of the thoracoabdominal aorta. 1993;105:557-8 (Letter)

Agnew R (see Browdie et al). 1993;105:732-6

Akiba T, Neirotti R, Becker AE. Is there an anatomic basis for subvalvular right ventricular outflow tract obstruction after an arterial switch repair for complete transposition? A morphometric study and review. 1993;105:142-6

Akins C. Discussion of Allen et al. 1993;105:864-84

Albain KS (see Rusch et al). 1993;105:97-106

Alladine MF (see Davies et al). 1993;105:979-87

Allen BS, Buckberg GD, Fontan FM, Kirsh MM, Popoff G, Beyersdorf F, Fabiani J-N, Acar C. Superiority of controlled surgical reperfusion versus percutaneous transluminal coronary angioplasty in acute coronary occlusion. 1993;105:864-84

Allen MS, Trastek VF, Deschamps C, Pairolero PC. Intrathoracic stomach: presentation and results of operation. 1993;105:253-9

Althaus U (see Inderbitzi et al). 1993; 105:84-8

Altorki NK, Sunagawa M, Skinner DB. Thoracic esophageal diverticula: Why is operation necessary? 1993;105:260-4

Alvarez-Sala R (see García-Talavera et al). 1993;105:767 (Letter)

Aly HM (see Abd-Elfattah et al). 1993;105:1095-105

Amato JJ. Discussion of Bove et al. 1993;105:1057-66

- Discussion of Laborde et al. 1993;105:278-80

Åm Holen E (see Øvrum et al). 1993;105:78-83

Amiral J (see Berruyer et al). 1993; 105:892-7

Amodeo A (see Di Donato et al). 1993;105:398-405

Anderson RP. Discussion of Yokoyama et al. 1993;105:912-7

- , Guyton SW, Paull DL, Tidwell SL. Selection of patients for same-day coronary bypass operations. 1993; 105:444-52

Andrews CW Jr (see Streitz et al). 1993;105:383-8

Angell WW. Discussion of Galloway et al. 1993;105:781-90

Antunes MJ. Transposition of the great arteries with posterior aorta. 1993;105:369 (Letter)

*January, 1-192: February, 193-382: March, 383-578: April, 579-772: May, 773964: June, $965-1160$.
Aoki M, Nomura F, Kawata H, Mayer JE Jr. Effect of calcium and preischemic hypothermia on recovery of myocardial function after cardioplegic ischemia in neonatal lambs. 1993; 105:207-13

Aoki M (see Inui et al). 1993;105:474-9

Appleyard RF (see Byme et al). 1993;105:689-93

Arciniegas E (see Hackbarth et al). 1993;105:31-6

Armiger LC (see Kadoba et al). 1993;105:132-41

Armitage JM, Fricker FJ, del Nido P, Starzl TE, Hardesty RL, Griffith BP. A decade (1982 to 1992) of pediatric cardiac transplantation and the impact of FK 506 immunosuppression. 1993;105:464-73

- - Kurland G, Hardesty RL, Michaels M, Morita S, Starzl TE, Yousem SA, Jaffe R, Griffith BP. Pediatric lung transplantation: the years 1985 to 1992 and the clinical trial of FK 506. 1993; 105:337-46

- (see Hardesty et al). 1993; 105:660-6

Aronson S, Lee BK, Zaroff JG, Wiencek JG, Walker R, Feinstein S, Karp RB. Myocardial distribution of cardioplegic solution after retrograde delivery in patients undergoing cardiac surgical procedures. 1993; 105:214-21

Arsiwala S, Panday S. A variant course of posterior descending artery. 1993; 105:952-3 (Letter)

Asai T (see Galloway et al). 1993;105:781-90

Asimacopoulos PJ, Camel JE, Iliopoulos DC, Reardon MJ. Blunt injury of the innominate artery: use of a vascular pericardial flap. 1993; 105:764-5 (Letter)

Asoh H (see Ichinose et al). 1993;105:1041-6

Attar S. Discussion of Altorki et al. 1993;105:260-4

-. Discussion of Bidstrup et al. 1993;105:147-53

Auer JE (see Hazelrigg et al). 1993; 105:389-93

Auge D, Georg R. Intrathoracic meningocele. 1993;105:561-2 (Letter)

Augustine J (see Caspi et al). 1993;105:59-67

- (see Caspi et al). 1993; 105:525-31

Avkiran M (see Yamamoto and Avkiran). 1993;105:120-31

\section{B}

Backer CL (see Muster et al). 1993;105:112-9

Badier M (see Massard et al). 1993;105:9-14

Bailey LL. Discussion of Armitage et al. 1993;105:464-73

—, Gundry SR, Razzouk AJ, Wang N, Sciolaro CM, Chiavarelli M, Loma Linda University Pediatric Heart Transplant Group. Bless the babies: one hundred fifteen late survivors of heart transplantation during the first year of life. 1993;105:805-15

Bains MS (see Burt et al). 1993; 105:89-96

Bakker PFA, Vermeulen FEE, de Boo JAJ, Elbers HRJ, van der Tweel I, van Beyeren I, Duyff P, Borst C, Robles de Medina EO, Tuntelder JR. Extensive cryoablation of the left ventricular posterior papillary muscle and subjacent ventricular wall: impact on mitral valve function and hemodynamics. 1993;105:327-36

Baktiroğlu S (see İğci et al). 1993;105:1116-8 (Letter)

Baldwin JC (see Higgins et al). 1993;105:965-71 
Ban T (see Oda et al). 1993;105:68-77

Bankoff MS (see Daly et al). 1993;105:904-11

Barankay A (see Dietrich et al). 1993;105:712-20

Barbero-Marcial M. Discussion of Bove et al. 1993;105:1057-66

Bamard PM (see Knott-Craig et al). 1993;105:394-7

Bamer HB (see Fischer and Bamer). 1993;105:762-3 (Letter)

Barratt-Boyes B. Discussion of Clarke et al. 1993;105:934-42

Bartlett RH (see Plötz et al). 1993;105:823-32

Bastien O (see Berruyer et al). 1993;105:892-7

Batisse A (see Laborde et al). 1993;105:278-80

Baumann FG (see Galloway et al). 1993;105:781-90

Baylen BG (see Myers et al). 1993;105:281-8

Beardmore H (see Browdie et al). 1993;105:732-6

Becker AE (see Akiba et al). 1993;105:142-6

Becker V (see Inui et al). 1993;105:474-9

Beekman RH III (see Bove et al). 1993;105:289-96 (see Bove et al). 1993; 105:1057-66

Belleville J (see Berruyer et al). 1993;105:892-7

Benfield JR. Discussion of Rusch et al. 1993;105:97-106

_. Discussion of Streitz et al. 1993;105:383-8

—. Discussion of Wolfe et al. 1993;105:749-56

—. Discussion of Yokoyama et al. 1993;105:912-7

- (see Rusch et al). 1993;105:97-106

Benson LN (see Caspi et al). 1993;105:59-67

- (see Caspi et al). 1993; 105:525-31

Berman K (see Ko et al). 1993; 105:1015-24

Bernard S (see Matsuura et al). 1993;105:45-51

Bemhard A (see Sievers et al). 1993;105:580-90

- (see Sievers et al). 1993; 105:775-80

Berri G, Welsh P, Capelli H. Aortic aneurysm after subclavian arterial flap angioplasty for coarctation of the aorta. 1993;105:951 (Letter)

Berruyer M, Amiral J, Ffrench P, Belleville J, Bastien O, Clerc J, Kassir A, Estanove S, Dechavanne M. Immunization by bovine thrombin used with fibrin glue during cardiovascular operations: development of thrombin and factor $V$ inhibitors. 1993;105:892-7

Beyersdorf F (see Allen et al). 1993; 105:864-84

Bidstrup BP. Discussion of Tobe et al. 1993;105:1007-14

- Underwood SR, Sapsford RN, Streets EM. Effect of aprotinin (Trasylol) on aorta-coronary bypass graft patency. 1993;105:147-53

Bishop DA (see Clarke et al). 1993;105:934-42

Björk S (see Gatzinsky et al). 1993;105:553-5 (Letter)

__ (see Gatzinsky et al). 1993;105:556-7 (Letter reply)

Blackstone EH (see Hanley et al). 1993; 105:406-27

Bloch G (see Menasché et al). 1993;105:353-63

Boban M, Stowe DF, Kampine JP, Goldberg AH, Bosnjak ZJ. Effects of 2,3-butanedione monoxime in isolated hearts: protection during reperfusion after global ischemia. 1993;105:532-40

Bolam DL (see Leuschen et al). 1993; 105:885-91

Boldt J. Invited letter conceming: aprotinin use in pediatric cardiac operations. 1993; 105:759-60 (Letter reply)

- Knothe C, Zickmann B, Wege N, Dapper F, Hempelmann G. Comparison of two aprotinin dosage regimens in pediatric patients having cardiac operations: influence on platelet function and blood loss. 1993; 105:705-11

Bolling SF (see Ning et al). 1993;105:541-9

Bolman RM III. Discussion of Armitage et al. 1993;105:337-46

Borleffs JCC (see Wijngaard et al). 1993; 105:183-4 (Letter)

Bors V (see Jault et al). 1993;105:1 106-13

Borst C (see Bakker et al). 1993; 105:327-36

Borst HG (see Aebert et al). 1993;105:557-8 (Letter)

- (see Inui et al). 1993; 105:474-9

Bosnjak ZJ (see Boban et al). 1993;105:532-40

Bourel P (see Laborde et al). 1993;105:278-80

Bove EL. Discussion of Myers et al. 1993;105:281-8
-, Lupinetti FM, Pridjian AK, Beekman RH III, Callow LB, Snider AR, Rosenthal A. Results of a policy of primary repair of truncus arteriosus in the neonate. 1993;105:1057-66

—, Minich LL, Pridjian AK, Lupinetti FM, Snider AR, Dick M II, Beekman RH III. The management of severe subaortic stenosis, ventricular septal defect, and aortic arch obstruction in the neonate. 1993; 105:289-96

Bozfakioğlu Y (see ľgci et al). 1993; 105:1116-8 (Letter)

Brezina A (see Caspi et al). 1993;105:59-67

- (see Caspi et al). 1993;105:525-31

Browdie D, Todd D, Agnew R, Rosen W, Beardmore $H$. The use of "nonanatomic" pulmonary resection in infants with extensive congenital adenomatoid malformation of the lung. 1993;105:732-6

Brown JW (see Hartman et al). 1993;105:743-8

Brown R (see Adoumie et al). 1993;105:229-33

Bruniaux J (see Planché et al). 1993;105:925-33

Buckberg GD (see Allen et al). 1993;105:864-84

- (see Matheis et al). 1993; 105:513-9

Burkart PT (see Scharfman et al). 1993;105:765-6 (Letter)

Burt M, Karpeh M, Ukcha O, Bains MS, Martini N, McCormack PM, Rusch VW, Ginsberg RJ. Medical tumors of the chest wall: solitary plasmacytoma and Ewing's sarcoma. 1993;105:89-96

Bussey ME (see Leuschen et al). 1993;105:885-91

Butler J, Pillai R, Rocker GM, Westaby S, Parker D, Shale DJ. Effect of cardiopulmonary bypass on systemic release of neutrophil elastase and tumor necrosis factor. 1993;105:25-30

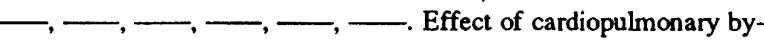
pass on systemic release of neutrophil elastase and tumor necrosis factor (1993;105:25-30). 1993;105:1056 (Correction)

Byme JG, Appleyard RF, Sun S-C, Couper GS, Sloane JA, Laurence RG, Cohn LH. Cardiac-derived thromboxane $A_{2}$ : An initiating mediator of reperfusion injury? 1993;105:689-93

\section{C}

Caballero P (see García-Talavera et al). 1993;105:767 (Letter)

Cabrol C (see Jault et al). 1993; 105:1106-13

Cachera JP. Discussion of Lytle et al. 1993;105:605-14

Callow LB (see Bove et al). 1993; 105:1057-66

Camel JE (see Asimacopoulos et al). 1993; 105:764-5 (Letter)

Campanile F (see Scalia et al). 1993; 105:633-42

Campbell DN (see Clarke et al). 1993;105:934-42

Canver CC. Isolated unilateral pulmonary artery agenesis. 1993;105: 766-7 (Letter)

Capelli H (see Berri et al). 1993;105:951 (Letter)

Cappelman WF (see Robicsek et al). 1993; 105:949-51 (Letter)

Carpentier A. Discussion of Castro et al. 1993;105:643-59

-Discussion of Llaneras et al. 1993; 105:439-43

Carrel T (see Pasic et al). 1993;105:321-6

Carson S (see Yau et al). 1993;105:833-44

Casarotto D (see Scalia et al). 1993;105:633-42

Caspi J, Coles JG, Benson LN, Herman SL, Augustine J, Tsao P, Brezina A, Kolin A, Wilson GJ. Effects of high plasma epinephrine and $\mathrm{Ca}^{2+}$ concentrations on neonatal myocardial function after ischemia. 1993; 105:59-67

,,--- Diaz RJ, Augustine J, Brezina A, Kolin A, Wilson GJ. The protective effect of magnesium on acute catecholamine cardiotoxicity in the neonate. 1993;105:525-31

Castaneda AR. Discussion of Bove et al. 1993;105:1057-66 - (see Hanley et al). 1993; 105:1047-56

Castle LW (see McCarthy et al). 1993;105:1077-87

Castro LJ, Moon MR, Rayhill SC, Niczyporuk MA, Ingels NB Jr, Daughters GT III, Derby GC, Miller DC. Annuloplasty with flexible or rigid ring does not alter left ventricular systolic performance, energetics, or ventricular-arterial coupling in conscious, closed-chest dogs. 1993;105:643-59

Causs T (see Fradin et al). 1993;105:364-5 (Letter) 
Cerrina J (see Dartevelle et al). 1993;105:1025-34

Chambers DJ (see Galiñanes et al). 1993;105:180-3 (Letter)

Chambers JS. Discussion of Hartman et al. 1993;105:743-8

- Discussion of Rusch et al. 1993;105:97-106

Chandrasekhar N (see Gokhale et al). 1993;105:557 (Letter)

Chang P (see Gates et al). 1993; 105:845-53

- (see Pearl et al). 1993;105:201-6

Chapelier AR (see Dartevelle et al). 1993;105:1025-34

Chard RB. Discussion of Myers et al. 1993;105:281-8

Chastre JC (see Jault et al). 1993; 105:1106-13

Chiavarelli M (see Bailey et al). 1993;105:805-15

Childs KF (see Ning et al). 1993;105:541-9

Chiu RC-J (see Adoumie et al). 1993; 105:229-33

Chow AW (see Sett et al). 1993; 105:428-34

Clark RE (see Cmolik et al). 1993;105:222-8

- (see Hennein et al). 1993; 105:624-32

Clarke DR, Campbell DN, Hayward AR, Bishop DA. Degeneration of aortic valve allografts in young recipients. 1993;105:934-42

Clerc J (see Berruyer et al). 1993; 105:892-7

Cmolik BL, Spero JA, Magovem GJ, Clark RE, Reid C. Redo cardiac surgery: late bleeding complications from topical thrombininduced factor $V$ deficiency. 1993;105:222-8

Cobanoglu A. Discussion of Miller and DeHoyos. 1993;105:247-52

Cohn LH. Discussion of Okoshi et al. 1993;105:791-5 (see Byme et al). 1993;105:689-93

Coles JG (see Caspi et al). 1993;105:59-67

- (see Caspi et al). 1993;105:525-31

Colvin SB (see Galloway et al). 1993;105:781-90

Comas JV (see Planché et al). 1993;105:925-33

Connolly JE. Discussion of Allen et al. 1993;105:253-9

Conte B, Leitner J. Leiomyosarcoma of the lung. 1993;105:1119-20 (Letter)

Cook NR (see Hanley et al). 1993; 105:1047-56

Cook WA. Discussion of Altorki et al. 1993;105:260-4

- Discussion of Hartman et al. 1993; 105:743-8

Cooper JD. Discussion of Inui et al. 1993;105:474-9

_ (see Date et al). 1993;105:480-91

- (see Date et al). 1993; 105:492-501

Cooper JM (see Date et al). 1993; 105:480-91

- (see Date et al). 1993; 105:492-501

Corno A, Papa M, Santoro F, Zoia E. Aneurysm of the membranous ventricular septum in transposition of the great arteries. 1993;105: 369-71 (Letter)

—, Samaja M. The reoxygenation phenomenon. 1993;105:373 (Letter)

Cosgrove DM III (see Lytle et al). 1993;105:605-14

_ (see McCarthy et al). 1993; 105:1077-87

Couper GS (see Byme et al). 1993;105:689-93

Crasti B (see Rocco et al). 1993;105:944-5 (Letter)

Crescenzo DG (see Abd-Elfattah et al). 1993;105:1095-105

Crowley JJ (see Rusch et al). 1993;105:97-106

Culliford AT (see Galloway et al). 1993;105:781-90

Cyran SE (see Myers et al). 1993; 105:281-8

\section{D}

Daly BDT, Mueller JD, Faling L, Diehl JT, Bankoff MS, Karp DD, Rand WM. N2 lung cancer: outcome in patients with falsenegative computed tomographic scans of the chest. 1993;105:90411

Danielson GK (see Orszulak et al). 1993;105:302-12

Dapper F (see Boldt et al). 1993; 105:705-11

Dartevelle PG, Chapelier AR, Macchiarini P, Lenot B, Cerrina J, Ladurie FLR, Parquin FJF, Lafont D. Anterior transcervicalthoracic approach for radical resection of lung tumors invading the thoracic inlet. 1993;105:1025-34
—, Macchiarini P. Actuarial five-year survival estimates. 1993; 105:375-7 (Letter reply)

Date H, Matsumura A, Manchester JK, Cooper JM, Lowry OH, Cooper JD. Changes in alveolar oxygen and carbon dioxide concentration and oxygen consumption during lung preservation: the maintenance of aerobic metabolism during lung preservation. 1993; 105:492-501

$\longrightarrow, \ldots$, Obo H, Lima O, Cooper JM, Sundaresan S, Lowry $\mathrm{OH}$, Cooper JD. Evaluation of lung metabolism during successful twenty-four-hour canine lung preservation. 1993;105:480-91

Daughters GT III (see Castro et al). 1993;105:643-59

Davies SW, Duffy JP, Wickens DG, Underwood SM, Hill A, Alladine MF, Feneck RO, Dormandy TL, Walesby RK. Time-course of free radical activity during coronary artery operations with cardiopulmonary bypass. 1993;105:979-87

Deac R (see Llaneras et al). 1993;105:439-43

Deac RC. Discussion of Hennein et al. 1993;105:624-32

de Boo JAJ (see Bakker et al). 1993;105:327-36

De Buyzere M (see De Smet et al). 1993;105:946-7 (Letter)

Dechavanne M (see Bermyer et al). 1993;105:892-7

DeHoyos A (see Miller et al). 1993;105:247-52

Della Pona C (see Rocco et al). 1993;105:944-5 (Letter)

del Nido P (see Armitage et al). 1993;105:464-73 (see Jimenez et al). 1993; 105:988-94

de Mascarel A (see Fradin et al). 1993;105:364-5 (Letter)

Dembitsky WP. Discussion of McCarthy et al. 1993;105:1077-87

—. Discussion of Yokoyama et al. 1993;105:912-7

DeMeester TR (see Stein et al). 1993;105:107-11

Derby GC (see Castro et al). 1993;105:643-59

Dernevik L (see Gatzinsky et al). 1993;105:553-5 (Letter)

- (see Gatzinsky et al). 1993; 105:556-7 (Letter reply)

Derrick MJ (see Yokoyama et al). 1993;105:912-7

Deschamps C (see Allen et al). 1993; 105:253-9

Deshmukh DR (see Hackbarth et al). 1993;105:31-6

Deslauriers J. Discussion of Miller. 1993;105:347-52 - (see Grégoire et al). 1993; 105:918-24

De Smet H, Missault L, Provenier F, De Buyzere M, Duprez D. Transesophageal echography as an emergency diagnostic tool for acute aortic dissection. 1993;105:946-7 (Letter)

DeSmet J-M (see Orszulak et al). 1993;105:302-12

Diaz RJ (see Caspi et al). 1993;105:525-31

Díaz S (see García-Talavera et al). 1993;105:767 (Letter)

di Carlo DD (see Di Donato et al). 1993;105:398-405

Dick M II (see Bove et al). 1993; 105:289-96

Di Donato RM, Amodeo A, di Carlo DD, Galletti L, Rinelli G, Pasquini L, Marcelletti C. Staged Fontan operation for complex cardiac anomalies with subaortic obstruction. 1993;105:398-405

Diehl JT (see Daly et al). 1993;105:904-11

Dietrich W, Mössinger HJ. Invited letter conceming: aprotinin use in pediatric cardiac operations. 1993;105:758-9 (Letter reply)

,-- , Spannagl M, Jochum M, Wendt P, Barankay A, Meisner H, Richter JA. Hemostatic activation during cardiopulmonary bypass with different aprotinin dosages in pediatric patients having cardiac operations. 1993;105:712-20

Ding X (see Ning et al). 1993;105:541-9

Djuranović S (see Rakić and Djuranović). 1993;105:1122 (Letter)

Domkowski PW (see Abd-Elfattah et al). 1993;105:1095-105

Dong FB (see Evans et al). 1993; 105:972-8

Dormandy TL (see Davies et al). 1993;105:979-87

Doty DB. Discussion of Myers et al. 1993;105:281-8

Discussion of Yau et al. 1993;105:833-44

Downing SW (see Llaneras et al). 1993;105:439-43

Dreyer WJ, Smith CW, Entman ML. Reply to: neutrophil activation during cardiopulmonary bypass (J THORAC CARDIOVASC SURG 1992;104:1746-8). 1993; 105:763 (Letter)

Drinkwater DC. Discussion of Di Donato et al. 1993;105:398-405 
- (see Gates et al). 1993;105:845-53

— (see Pearl et al). 1993;105:201-6

Duffy CE (see Muster et al). 1993; 105:112-9

Duffy JP (see Davies et al). 1993;105:979-87

Duhaylongsod FG (see Wolfe et al). 1993;105:749-56

Duprez D (see De Smet et al). 1993;105:946-7 (Letter)

Duran C. Discussion of Castro et al. 1993;105:643-59

du Toit EF (see Owen et al). 1993;105:995-1006

Duyff P (see Bakker et al). 1993; 105:327-36

\section{E}

Edmunds LH Jr. Discussion of Cmolik et al. 1993;105:222-8

—. Invited letter conceming: aprotinin use in pediatric cardiac operations. 1993;105:757-8 (Letter)

- (see Llaneras et al). 1993;105:439-43

Eisen RN (see Higgins et al). 1993;105:965-71

Elbers HRJ (see Bakker et al). 1993;105:327-36

Elkins RC (see Hedrick et al). 1993; 105:297-301

Elliott M (see Finn et al). 1993; 105:234-41

(see Miller et al). 1993;105:550-1 (Letter)

Ellis FH Jr. Discussion of Allen et al. 1993;105:253-9

—. Invited letter concerning: technique for prevention of gastroesophageal reflux after transthoracic Heller's operation. 1993; 105:555-6 (Letter)

- (see Streitz et al). 1993; 105:383-8

Endres S (see Markewitz et al). 1993;105:15-24

Engelman RM. Discussion of Markewitz et al. 1993;105:15-24

Entman ML (see Dreyer et al). 1993;105:763 (Letter)

Ercan E (see İgci et al). 1993;105:1116-8 (Letter)

Estanove S (see Berruyer et al). 1993;105:892-7

Evans RW, Manninen DL, Dong FB. An economic analysis of heart-lung transplantation: costs, insurance coverage, and reimbursement. 1993;105:972-8

\section{$\mathbf{F}$}

Faber LP. Discussion of Altorki et al. 1993;105:260-4

- Discussion of Burt et al. 1993;105:89-96

_. Discussion of Daly et al. 1993;105:904-11

—. Discussion of Hartman et al. 1993;105:743-8

_. Discussion of Rusch et al. 1993;105:97-106

Fabiani J-N (see Allen et al). 1993; 105:864-84

Fahey AL (see Ko et al). 1993;105:1015-24

Faist E (see Markewitz et al). 1993;105:15-24

Faling LJ (see Daly et al). 1993;105:904-11

Fasoli G (see Scalia et al). 1993; 105:633-42

Feinberg H (see Jimenez et al). 1993;105:988-94

Feinstein S (see Aronson et al). 1993;105:214-21

Feneck RO (see Davies et al). 1993;105:979-87

Fenton KN, Heinemann MK, Hanley FL. Exclusion of the placenta during fetal cardiac bypass augments systemic flow and provides important information about the mechanism of placental injury. 1993; 105:502-12

Ferraris V (see Scharfman et al). 1993;105:765-6 (Letter)

Ffrench P (see Berruyer et al). 1993; 105:892-7

Finn A, Naik S, Klein N, Levinsky RJ, Strobel S, Elliott M. Interleukin-8 release and neutrophil degranulation after pediatric cardiopulmonary bypass. 1993;105:234-41

Firmin RK (see Underwood et al). 1993;105:377-8 (Letter)

Fischer V, Barner HB. Reply to invited letter concerning: assessment of myocardial ultrastructure after retrograde infusion of cardioplegic solution (J THORAC CARDrOVASC SURG 1992;104:1745-6). 1993; 105:762-3 (Letter)

Flameng W (see Noyez et al). 1993;105:854-63

Fleisher AG (see Wemer et al). 1993;105:737-42
Flomenbaum MA, Schoen FJ. Effects of fixation back pressure and antimineralization treatment on the morphology of porcine aortic bioprosthetic valves. 1993; 105:154-64

Foker JE. Discussion of Hanley et al. 1993;105:406-27

Follette DM. Discussion of Allen et al. 1993;105:864-84

-. Discussion of Acki et al. 1993;105:207-13

Fontan FM (see Allen et al). 1993;105:864-84

- (see Fradin et al). 1993;105:364-5 (Letter)

Fosburg RG. Fulfilling expectations. 1993;105:194-200 (Pres. address)

Fradin D, Causs T, Rabaud M, de Mascarel A, Fontan F. Preliminary experimental results of a new resorbable biomaterial as pericardial substitute. 1993;105:364-5 (Letter)

Franco KL (see Higgins et al). 1993; 105:965-71

Frater RW. Discussion of Llaneras et al. 1993;105:439-43

Freedom RM. Invited letter conceming: critical aortic stenosis. 1993; 105:366-7 (Letter reply)

- (see Hanley et al). 1993; 105:406-27

Fricker FJ (see Armitage et al). 1993;105:337-46

- (see Armitage et al). 1993;105:464-73

Fuchs D (see Markewitz et al). 1993;105:15-24

Fukuda S (see Suma et al). 1993;105:615-23

Furrer M (see Inderbitzi et al). 1993;105:84-8

Furuta S (see Suma et al). 1993;105:615-23

$G$

Gaither JM (see Hartman et al). 1993;105:743-8

Galiñanes M, Chambers DJ, Hearse DJ. Should adenosine continue to be ignored as a cardioprotective agent in cardiac operations? 1993; 105:180-3 (Letter)

Gallagher KP (see Ning et al). 1993;105:541-9

Galletti L (see Di Donato et al). 1993;105:398-405

Galletti PM (see Okoshi et al). 1993;105:791-5

Galloway AC, Colvin SB, Grossi EA, Parish MA, Culliford AT, Asai T, Rofsky NM, Weinreb JC, Shapiro S, Baumann FG, Spencer FC. Surgical repair of type A aortic dissection by the circulatory arrest-graft inclusion technique in sixty-six patients. 1993;105: $781-90$

Gandjbakhch I (see Jault et al). 1993;105:1106-13

Garan H (see Mitchell et al). 1993;105:453-63

García-Talavera I, Alvarez-Sala R, Caballero P, Díaz S, Villamor J. Pleural effusion as the first manifestation of a malignant fibrous histiocytoma. 1993;105:767 (Letter)

Gates RN, Laks H, Drinkwater DC, Pearl J, Zaragoza AM, Kaczer E, Chang $P$. The microvascular distribution of cardioplegic solution in the piglet heart: retrograde versus antegrade delivery. 1993;105: 845-53

- (see Pearl et al). 1993;105:201-6

Gatzinsky P, Dernevik L, Björk S, Sandberg N. Technique for prevention of gastroesophageal reflux after transthoracic Heller's operation. 1993; 105:553-5 (Letter)

,,-- Sandberg N, Björk S. Invited letter conceming: technique for prevention of gastroesophageal reflux after transthoracic Heller's operation. 1993;105:556-7 (Letter reply)

Georg R (see Auge and Georg). 1993;105:561-2 (Letter)

George JF. Invited letter concerning: transplantation tolerance and transplantation. 1993;105:184-5 (Letter)

Giben C (see Jault et al). 1993;105:1106-13

Gillis B (see Tobe et al). 1993;105:1007-14

Gimbrère J (see Noyez et al). 1993;105:854-63

Ginsberg RJ. Discussion of Dartevelle et al. 1993;105:1025-34

- Discussion of Orringer et al. 1993;105:265-77

(see Burt et al). 1993;105:89-96

Giudicelli R (see Massard et al). 1993;105:9-14

Gleason MM (see Myers et al). 1993;105:281-8

Gmelig Meyling FHJ (see Schuurman et al). 1993;105:178-80 (Letter) 
- (see Wijngaard et al). 1993;105:183-4 (Letter)

_- (see Wijngaard et al). 1993;105:185 (Letter reply)

Goddard M (see Okoshi et al). 1993;105:791-5

Gokhale AGK, Suhasini T, Saraswati V, Chandrasekhar N, Rajagopal P. Cold agglutinins and warm heart surgery. 1993;105:557 (Letter)

Goldberg AH (see Boban et al). 1993;105:532-40

Goodrich PD (see Leuschen et al). 1993;105:885-91

Goor DA (see Mohr et al). 1993;105:371-3 (Letter)

Goormastic M (see Lytle et al). 1993;105:605-14

Gorczynski RM (see Hirai et al). 1993;105:1-8

Goto K (see Onizuka et al). 1993;105:559-60 (Letter)

Goto Y (see Yaku et al). 1993;105:679-88

Gøtzsche LB-H, Weeke J. Reply to invited letter conceming: changes in plasma-free thyroid hormones during cardiopulmonary bypass (J Thorac Cardovasc SuRg 1992;104:526-7). 1993;105:176-8 (Letter)

Graham TR (see Underwood et al). 1993;105:377-8 (Letter)

Grégoire J, Deslauriers J, Guojin L, Rouleau J. Indications, risks, and results of completion pneumonectomy. 1993;105:918-24

Griffin BR (see Rusch et al). 1993;105:97-106

Griffith BP. Discussion of Pierce et al. 1993;105:520-4

- (see Armitage et al). 1993;105:337-46

- (see Armitage et al). 1993;105:464-73

- (see Hardesty et al). 1993;105:660-6

Grossi EA (see Galloway et al). 1993;105:781-90

Grousset C (see Menasché et al). 1993;105:353-63

Guha M (see Sievers et al). 1993;105:775-80

Guillot C (see Massard et al). 1993;105:9-14

Gundry SR (see Bailey et al). 1993;105:805-15

Guojin L (see Grégoire et al). 1993;105:918-24

Guyton RA. Discussion of Allen et al. 1993;105:864-84

- Discussion of Markewitz et al. 1993;105:15-24

Guyton SW. Discussion of Mitchell et al. 1993;105:453-63

- (see Anderson et al). 1993; 105:444-52

H

Hackbarth R, Sarnaik AP, Meert K, Deshmukh DR, Arciniegas E. Changes in plasma fibronectin in children after elective repair of congenital heart defects. 1993; 105:31-6

Hammond GL (see Higgins et al). 1993;105:965-71

Haneda K (see Sato et al). 1993;105:313-20

Hanley FL, Heinemann MK, Jonas RA, Mayer JE Jr, Cook NR, Wessel DL, Castaneda AR. Repair of truncus arteriosus in the neonate. 1993; 105:1047-56

—, Sade RM, Blackstone EH, Kirklin JW, Freedom RM, Nanda NC. Outcomes in neonatal pulmonary atresia with intact ventricular septum: a multiinstitutional study. 1993;105:406-27

- (see Fenton et al). 1993;105:502-12

Hara N (see Ichinose et al). 1993;105:1041-6

Harbold NB Jr (see Robicsek et al). 1993;105:949-51 (Letter)

Hardesty RL, Aeba R, Armitage JM, Kormos RL, Griffith BP. A clinical trial of University of Wisconsin solution for pulmonary preservation. 1993; 105:660-6

- (see Armitage et al). 1993;105:337-46

_- (see Amitage et al). 1993;105:464-73

Harlan BJ. Discussion of Yokoyama et al. 1993;105:912-7

Hartman DL, Gaither JM, Kesler KA, Mylet DM, Brown JW, Mathur PN. Comparison of insufflated talc under thoracoscopic guidance with standard tetracycline and bleomycin pleurodesis for control of malignant pleural effusions. 1993;105:743-8

Hasegawa T (see Misawa et al). 1993;105:168-72

Hastings JC III, Robicsek F. Clinical significance of epicardial pacing wire cultures. 1993;105:165-7

Hathorn JW (see Wolfe et al). 1993;105:749-56

Haverich A (see Inui et al). 1993;105:474-9
Hayashida N (see Isomura et al). 1993;105:1088-94

Hayward AR (see Clarke et al). 1993;105:934-42

Hazelrigg SR, Landreneau RJ, Mack M, Acuff T, Seifert PE, Auer JE, Magee M. Thoracoscopic stapled resection for spontaneous pneumothorax. 1993; 105:389-93

Hearse DJ (see Galiñanes et al). 1993;105:180-3 (Letter)

Hedrick M, Elkins RC, Knott-Craig CJ, Razook JD. Successful thrombectomy for thrombosis of the right side of the heart after the Fontan operation: report of two cases and review of the literature. 1993;105:297-301

Hefner LL (see Urthaler et al). 1993;105:1114-6 (Letter)

Heinemann MK (see Fenton et al). 1993;105:502-12

- (see Hanley et al). 1993;105:1047-56

Heintzen PH (see Sievers et al). 1993;105:580-90

Hempelmann G (see Boldt et al). 1993;105:705-11

Hendry PJ, Labow RS, Keon WJ. A comparison of intracellular solutions for donor heart preservation. 1993; 105:667-73

Hennein HA, Jones M, Stone CD, Clark RE. Left ventricular function in experimental mitral regurgitation with intact chordae tendineae. 1993;105:624-32

Herman SL (see Caspi et al). 1993; 105:59-67

- (see Caspi et al). 1993;105:525-31

Higgins RSD, Letsou GV, Sanchez JA, Eisen RN, Smith GJW, Franco KL, Hammond GL, Baldwin JC. Improved ultrastructural lung preservation with prostaglandin $E_{1}$ as donor pretreatment in a primate model of heart-lung transplantation. 1993;105:965-71

Hill A (see Davies et al). 1993;105:979-87

Hirai T, Waddell TK, Puskas JD, Wada H, Hitomi S, Gorczynski RM, Slutsky AS, Patterson GA. Prolonged lung allograft survival with a short course of FK 506. 1993;105:1-8

Hirano A (see Isomura et al). 1993;105:1088-94

Hisatomi K (see Isomura et al). 1993;105:1088-94

Hitomi S (see Hirai et al). 1993;105:1-8

Hoeft S (see Stein et al). 1993;105:107-11

Hofer BO (see Tobe et al). 1993;105:1007-14

Hoie EB (see Leuschen et al). 1993;105:885-91

Hop WCJ (see Tilanus et al). 1993; 105:898-903

Hopkins RA (see Abd-Elfattah et al). 1993;105:1095-105

Hori M (see Onizuka et al). 1993;105:559-60 (Letter)

Hoshino S (see Yamagishi et al). 1993;105:1067-76

Hu W-c (see Spahn et al). 1993;105:694-704

Hudon MPJ (see Sett et al). 1993;105:428-34

Hung J (see Lam et al). 1993;105:1035-40

I

Ichikawa H, Yamamoto F. Proton gradient during cardiac arrest: oxygenation of St. Thomas' Hospital cardioplegic solution and carbon dioxide level. 1993; 105:551-2 (Letter)

Ichinose Y, Hara N, Ohta M, Asoh H, Yano T, Maeda K, Yagawa K. Hypotonic cisplatin treatment for carcinomatous pleuritis found at thoracotomy in patients with lung cancer: in vitro experiments and preliminary clinical results. 1993;105:1041-6

İgci A, Kalayci G, Baktiroğlu S, Bozfakioglu Y, Ercan E, Müslümanogłlu M. Dysphagia lusoria. 1993;105:1116-8 (Letter)

Ihnken K (see Matheis et al). 1993;105:513-9

Ikonomidis JS (see Yau et al). 1993;105:833-44

Ilbawi MN (see Muster et al). 1993; 105:112-9

Iliopoulos DC (see Asimacopoulos et al). 1993;105:764-5 (Letter)

Imai Y (see Yamagishi et al). 1993;105:1067-76

Inderbitzi RGC, Furrer M, Striffeler H, Althaus U. Thoracoscopic pleurectomy for treatment of complicated spontaneous pneumothorax. 1993;105:84-8

Ingels NB Jr (see Castro et al). 1993;105:643-59 
Inui K, Schäfers H-J, Aoki M, Becker V, Ongsiek B, Kemnitz J, Haverich A, Borst HG. Bronchial circulation after experimental lung transplantation: the effect of long-term administration of prednisolone. 1993;105:474-9

Ishihara K (see Yamagishi et al). 1993; 105:1067-76

Isom OW (see Ko et al). 1993;105:1015-24

Isomura T, Hisatomi $K$, Hirano A, Hayashida N, Matsuzoe S, Ohishi $K$. Clinical evaluation with exercise performance in twenty patients who underwent coronary artery bypass grafting with both the gastroepiploic and intemal thoracic arteries. 1993;105:108894

Itoh T (see Sato et al). 1993;105:313-20

Ivanov J (see Yau et al). 1993;105:833-44

Jaffe R (see Armitage et al). 1993; 105:337-46

Jambroes G (see Wijngaard et al). 1993;105:183-4 (Letter)

James J (see Miller et al). 1993;105:550-1 (Letter)

Jamieson WRE (see Sett et al). 1993;105:428-34

Jansen EWL (see Schuurman et al). 1993;105:178-80 (Letter)

Jault F, Gandjbakhch I, Chastre JC, Levasseur JP, Bors V, Gibert C, Pavie A, Cabrol C. Prosthetic valve endocarditis with ring abscesses: surgical management and long-term results. 1993;105: 1106-13

Jebara V. Discussion of Bidstrup et al. 1993; 105:147-53

Jimenez E, del Nido P, Feinberg H, Levitsky S. Redistribution of myocardial calcium during ischemia: relationship to onset of contracture. 1993;105:988-94

Jochum M (see Dietrich et al). 1993; 105:712-20

John LCH, Rees GM, Kovacs IB. Inhibition of platelet function by heparin: an etiologic factor in postbypass hemorthage. 1993;105: 816-22

Johnson H (see Pinkard et al). 1993;105:721-8

Jonas RA. Invited letter conceming: the importance of pulsatile flow when systemic venous return is connected directly to the pulmonary arteries. 1993; 105:173-5 (Letter)

Jonas RA (see Hanley et al). 1993; 105:1047-56

- (see Kadoba et al). 1993;105:132-41

Jones JL (see Abd-Elfattah et al). 1993;105:1095-105

Jones K. Discussion of Anderson et al. 1993;105:444-52

- Discussion of Fenton et al. 1993;105:502-12

Jones M (see Hennein et al). 1993;105:624-32

Joubert JR (see Knott-Craig et al). 1993;105:394-7

Kaczer E (see Gates et al). 1993;105:845-53

Kadoba K, Armiger LC, Sawatari K, Jonas RA. Mechanical durability of pulmonary allograft conduits at systemic pressure: angiographic and histologic study in lambs. 1993;105:132-41

Kalayci G (see lğci et al). 1993;105:1116-8 (Letter)

Kampine JP (see Boban et al). 1993;105:532-40

Karam J (see Laborde et al). 1993;105:278-80

Karp DD (see Daly et al). 1993;105:904-11

Karp RB (see Aronson et al). 1993; 105:214-21

Karpeh M (see Burt et al). 1993;105:89-96

Kassir A (see Bernuyer et al). 1993; 105:892-7

Kato M (see Misawa et al). 1993;105:168-72

Kawaguchi O (see Yaku et al). 1993; 105:679-88

Kawashima Y, Matsuda H, Yagihara T, Shimazaki Y, Yamamoto F, Nishigaki $K$, Miura $T$, Uemura $H$. Intraventricular repair for Taussig-Bing anomaly. 1993;105:591-7

Kawata H (see Aoki et al). 1993;105:207-13

Kay G. Discussion of Yau et al. 1993;105:833-44

Kemnitz J (see Inui et al). 1993;105:474-9

Kennedy JH. Discussion of Matheis et al. 1993;105:513-9

Keon WJ (see Hendry et al). 1993;105:667-73
Kesler KA (see Hartman et al). 1993;105:743-8

Khalil-Marzouk JF. Allograft replacement of the trachea: experimental synchronous revascularization of composite thyrotracheal transplant. 1993; 105:242-6

Kimura H (see Koike and Kimura). 1993;105:1121-2 (Letter)

Kirklin JW (see Hanley et al). 1993; 105:406-27

Kirschner PA. Discussion of Miller. 1993;105:347-52

Kirsh MM (see Allen et al). 1993;105:864-84

Klein AL (see McCarthy et al). 1993;105:1077-87

Klein N (see Finn et al). 1993;105:234-41

Knape JTA (see Noyez et al). 1993;105:854-63

Knothe C (see Boldt et al). 1993;105:705-11

Knott-Craig CJ, Oostuizen JG, Rossouw G, Joubert JR, Bamard PM. Management and prognosis of massive hemoptysis: recent experience with 120 patients. 1993; 105:394-7

- (see Hedrick et al). 1993;105:297-301

Ko W, Zelano JA, Fahey AL, Berman K, Lang D, Isom OW, Krieger $\mathrm{KH}$. The effects of amrinone versus dobutamine on myocardial mechanics and energetics after hypothermic global ischemia. 1993;105:1015-24

Kobayashi T (see Tabata et al). 1993; 105:598-604

Koh Y (see Yamagishi et al). 1993;105:1067-76

Kohno T, Murakami T, Wakabayashi A. Anatomic lobectomy of the lung by means of thoracoscopy: an experimental study. 1993;105: 729-31

Koide M (see Yamagishi et al). 1993; 105:1067-76

Koike R, Kimura H. Dilating effects of isosorbide dinitrate on diameter of internal thoracic artery graft. 1993;105:1121-2 (Letter)

Kolff J. Discussion of Lytle et al. 1993;105:605-14

Kolin A (see Caspi et al). 1993;105:59-67

- (see Caspi et al). 1993;105:525-31

Kormos RL (see Hardesty et al). 1993;105:660-6

Kovacs IB (see John et al). 1993;105:816-22

Kreitmann B (see Metras et al). 1993;105:186-8 (Letter)

Krieger KH (see Ko et al). 1993;105:1015-24

Krukenkamp IB (see Misare et al). 1993;105:37-44

Kurland G (see Armitage et al). 1993;105:337-46

\section{$\mathbf{L}$}

Laas J (see Aebert et al). 1993;105:557-8 (Letter)

Laborde F, Noihomme P, Karam J, Batisse A, Bourel P, Saint Maurice $O$. A new video-assisted thoracoscopic surgical technique for interruption of patent ductus arteriosus in infants and children. 1993; 105:278-80

Labow RS (see Hendry et al). 1993;105:667-73

Lacour-Gayet F (see Planché et al). 1993;105:925-33

Lacquet LK (see Noyez et al). 1993;105:854-63 (see van Son et al). 1993;105:368-9 (Letter)

Ladurie FLR (see Dartevelle et al). 1993;105:1025-34

Lafont D (see Dartevelle et al). 1993;105:1025-34

Laks H (see Gates et al). 1993;105:845-53 (see Pearl et al). 1993;105:201-6

Lam S, MacAulay C, Hung J, LeRiche J, Profio AE, Palcic B. Detection of dysplasia and carcinoma in situ with a lung imaging fluorescence endoscope device. 1993;105:1035-40

Landreneau RJ. Discussion of Kohno et al. 1993;105:729-31

_ (see Hazelrigg et al). 1993; 105:389-93

Lang D (see Ko et al). 1993;105:1015-24

Lang S (see Markewitz et al). 1993;105:15-24

Lange PE (see Sievers et al). 1993; 105:580-90

Langenhorst BLAM (see Tilanus et al). 1993;105:898-903

Laurence RG (see Byme et al). 1993; 105:689-93

Lavee J (see Mohr et al). 1993; 105:371-3 (Letter)

Lazar HL. Discussion of Allen et al. 1993;105:864-84

- (see Matsuura et al). 1993; 105:45-51

Lazer ZP (see Misare et al). 1993;105:37-44 
LeBlanc JG (see Wemer et al). 1993;105:737-42

Lee AW (see Yokoyama et al). 1993;105:912-7

Lee BK (see Aronson et al). 1993; 105:214-21

Lee R (see Mitchell et al). 1993;105:453-63

Leitner J (see Conte and Leitner). 1993;105:1119-20 (Letter)

Lenot B (see Dartevelle et al). 1993;105:1025-34

Leone BJ (see Spahn et al). 1993; 105:694-704

Leopold KA (see Wolfe et al). 1993;105:749-56

LeRiche J (see Lam et al). 1993;105:1035-40

Letsou GV (see Higgins et al). 1993;105:965-71

Leuschen MP, Willett LD, Hoie EB, Bolam DL, Bussey ME, Goodrich PD, Zach TL, Nelson RM Jr. Plasma fentanyl levels in infants undergoing extracorporeal membrane oxygenation. 1993;105: 885-91

Levasseur JP (see Jault et al). 1993;105:1106-13

Levinsky RJ (see Finn et al). 1993;105:234-41

Levitsky S (see Jimenez et al). 1993;105:988-94

_- (see Misare et al). 1993;105:37-44

Lewis RJ. Mesothelioma: an incurable, nonsurgically treatable disease. 1993;105:943 (Letter)

Leyh R (see Sievers et al). 1993;105:775-80

Leyland SA (see Pinkard et al). 1993;105:721-8

Lima JAC (see Llaneras et al). 1993;105:439-43

Lima $O$ (see Date et al). 1993;105:480-91

Linden PL (see Llaneras et al). 1993;105:439-43

Liu P (see Yau et al). 1993; 105:833-44

Livingston RB (see Rusch et al). 1993;105:97-106

Llaneras MR, Nance ML, Streicher JT, Linden PL, Downing SW, Lima JAC, Deac R, Edmunds LH Jr. Pathogenesis of ischemic mitral insufficiency. 1993;105:439-43

Loma Linda University Pediatric Heart Transplant Group (see Bailey et al). 1993;105:805-15

Lonchyna V (see Rusch et al). 1993;105:97-106

Loop FD (see Lytle et al). 1993; 105:605-14

Loose R (see Sievers et al). 1993; 105:775-80

Lowe JE (see Spahn et al). 1993; 105:694-704

Lowry OH (see Date et al). 1993; 105:480-91

_- (see Date et al). 1993;105:492-501

Lupinetti FM (see Bove et al). 1993; 105:289-96

- (see Bove et al). 1993; 105:1057-66

Lytle BW. Discussion of Suma et al. 1993;105:615-23

—- Loop FD, Taylor PC, Goormastic M, Stewant RW, Novoa R, McCarthy P, Cosgrove DM, Schnauffer MJ. The effect of coronary reoperation on the survival of patients with stenoses in saphenous vein bypass grafts to coronary arteries. 1993;105:605-14

M

MacAulay C (see Lam et al). 1993;105:1035-40

Macchiarini P (see Dartevelle and Macchiarini). 1993;105:375-7 (Letter reply)

- (see Dartevelle et al). 1993;105:1025-34

Mack M (see Hazelrigg et al). 1993; 105:389-93

Maeda K (see Ichinose et al). 1993;105:1041-6

Magee M (see Hazelrigg et al). 1993;105:389-93

Magovem GJ (see Cmolik et al). 1993;105:222-8

Maloney JD (see McCarthy et al). 1993;105:1077-87

Manchester JK (see Date et al). 1993;105:480-91

- (see Date et al). 1993; 105:492-501

Manninen DL (see Evans et al). 1993;105:972-8

Marcelletti C (see Di Donato et al). 1993;105:398-405

Mark JBD. Discussion of Altorki et al. 1993;105:260-4

. Discussion of Daly et al. 1993;105:904-11

— Discussion of Hazelrigg et al. 1993;105:389-93

_. Discussion of Wolfe et al. 1993;105:749-56
Markewitz A, Faist E, Lang S, Endres S, Fuchs D, Reichart B. Successful restoration of cell-mediated immune response after cardiopulmonary bypass by immunomodulation. 1993;105:15-24

Marseille-Montreal lung transplant program (see Massard et al). 1993; 105:9-14

Marshall B (see Orringer et al). 1993;105:265-77

Martin GR, Short BL. The reoxygenation phenomenon. 1993;105: 373-4 (Letter reply)

Martini N (see Burt et al). 1993; 105:89-96

Masaki T (see Onizuka et al). 1993;105:559-60 (Letter)

Massard G, Badier M, Guillot C, Reynaud M, Thomas P, Giudicelli R, Noirclerc M, Marseille-Montreal lung transplant program. Lung size matching for double lung transplantation based on the submammary thoracic perimeter: accuracy and functional results. 1993;105:9-14

Matheis G, Tixier DB, Buckberg GD, Ihnken K, Morita K, Sherman MP, Young HH. Cardiopulmonary dysfunction produced by reoxygenation of immature hypoxemic animals supported by cardiopulmonary bypass: prevention by intravenous metabolic pretreatment. 1993; 105:513-9

Mathisen DJ. Discussion of Rusch et al. 1993;105:97-106

- Discussion of Stein et al. 1993;105:107-11

Mathur PN (see Hartman et al). 1993;105:743-8

Matos-Cruz M (see Robicsek et al). 1993;105:949-51 (Letter)

Matsuda H (see Kawashima et al). 1993;105:591-7

Matsumoto A (see Noishiki et al). 1993;105:796-804

Matsumura A (see Date et al). 1993; 105:480-91

- (see Date et al). 1993;105:492-501

Matsuno S (see Tabata et al). 1993;105:598-604

Matsuura H, Lazar HL, Yang X, Rivers S, Treanor P, Bemard S, Shemin RJ. Warm versus cold blood cardioplegia-Is there a difference? 1993;105:45-51

Matsuzoe S (see Isomura et al). 1993; 105:1088-94

Mavroudis C. Discussion of Bailey et al. 1993;105:805-15

_. Discussion of Hanley et al. 1993;105:406-27

- Discussion of Kawashima et al. 1993;105:591-7

_. Discussion of Laborde et al. 1993;105:278-80

- Discussion of Planché et al. 1993;105:925-33

- (see Muster and Mavroudis). 1993;105:175-6 (Letter reply)

- (see Muster et al). 1993;105:112-9

Mayer JE Jr (see Aoki et al). 1993; 105:207-13

- (see Hanley et al). 1993;105:1047-56

Mayumi H, Tokunaga K. Prostaglandin $E_{1}$ for patients who have both heart and lung failure after cardiotomy. 1993; 105:1120-1 (Letter)

Mazzucco A (see Scalia et al). 1993;105:633-42

McCarthy PM, Castle LW, Maloney JD, Trohman RG, Simmons TW, White RD, Klein AL, Cosgrove DM III. Initial experience with the maze procedure for atrial fibrillation. 1993; 105:1077-87

- (see Lytle et al). 1993; 105:605-14

McCormack PM (see Burt et al). 1993;105:89-96

McDougall JC (see Tazelaar et al). 1993;105:674-8

McGregor CGA (see Tazelaar et al). 1993;105:674-8

McKay R. Invited letter conceming: critical aortic stenosis. 1993;105: 365-6 (Letter)

McKenna R Jr (see Rusch et al). 1993;105:97-106

McKneally MF. Discussion of Daly et al. 1993;105:904-11

_. Discussion of Higgins et al. 1993;105:965-71

_. Discussion of Inui et al. 1993;105:474-9

—. Discussion of Khalil-Marzouk. 1993;105:242-6

McRae RL (see Spahn et al). 1993; 105:694-704

Meen K (see Hackbarth et al). 1993;105:31-6

Meisner H (see Dietrich et al). 1993;105:712-20

Melacini P (see Scalia et al). 1993;105:633-42

Menasché P, Pradier F, Grousset C, Peynet J, Mouas C, Bloch G, Piwnica A. Improved recovery of heart transplants with a specific kit of preservation solutions. 1993;105:353-63 
Meneshian A (see Pearl et al). 1993;105:201-6

Mengoli L. Actuarial five-year survival estimates. 1993;105:375 (Letter)

Menius AJ (see Spahn et al). 1993;105:694-704

Messier RH Jr (see Abd-Elfattah et al). 1993;105:1095-105

Metras DR. Discussion of Bove et al. 1993;105:289-96

- Kreitmann B, Tatou E, Riberi A, Wemert F. Tetralogy of Fallot with pulmonary atresia, coronary artery-pulmonary artery fistula, and origin of left pulmonary artery from descending aorta: total correction in infancy. 1993;105:186-8 (Letter)

Michaels M (see Armitage et al). 1993;105:337-46

Mickle DAG (see Yau et al). 1993;105:833-44

Milano A (see Scalia et al). 1993;105:633-42

Miller DC. Discussion of Galloway et al. 1993;105:781-90

- (see Castro et al). 1993;105:643-59

Miller JD, DeHoyos A, University of Toronto and Washington University Lung Transplant Programs. An evaluation of the role of omentopexy and of early perioperative corticosteroid administration in clinical lung transplantation. 1993; 105:247-52

Miller JI Jr. Physiologic evaluation of pulmonary function in the candidate for lung resection. 1993; 105:347-52

Miller OI, James J, Elliott MJ. Intraoperative use of inhaled low-dose nitric oxide. 1993; 105:550-1 (Letter)

Mills NL. Discussion of Suma et al. 1993;105:615-23

Minich LL (see Bove et al). 1993; 105:289-96

Misare BD, Krukenkamp IB, Lazer ZP, Levitsky S. Recovery of postischemic contractile function is depressed by antegrade warm continuous blood cardioplegia. 1993; 105:37-44

Misawa Y, Hasegawa T, Kato M. Clinical experience with the Omnicarbon prosthetic heart valve. 1993;105:168-72

Missault L (see De Smet et al). 1993;105:946-7 (Letter)

Mitchell JD, Lee R, Garan H, Ruskin JN, Torchiana DF, Vlahakes GJ. Experience with an implantable tiered therapy device incorporating antitachycardia pacing and cardioverter/defibrillator therapy. 1993; 105:453-63

Mitchell RL. Discussion of Kohno et al. 1993;105:729-31

—. Discussion of Wolfe et al. 1993;105:749-56

Mitsui K (see Onizuka et al). 1993;105:559-60 (Letter)

Miura M (see Sato et al). 1993; 105:313-20

Miura T (see Kawashima et al). 1993;105:591-7

Miyamoto A-T (see Oda et al). 1993;105:68-77

Miyauchi T (see Onizuka et al). 1993;105:559-60 (Letter)

Mohabeer MK (see Yau et al). 1993; 105:833-44

Mohr R, Sagi B, Lavee J, Goor DA. The hemostatic effect of autologous platelet-rich plasma versus autologous whole blood after cardiac operations: Is platelet separation really necessary? 1993; 105:371-3 (Letter)

Mohri H (see Sato et al). 1993;105:313-20

Moon MR (see Castro et al). 1993;105:643-59

Morgan M (see Pinkard et al). 1993;105:721-8

Mori A (see Tabata et al). 1993;105:598-604

Morita K (see Matheis et al). 1993;105:513-9

Morita S (see Armitage et al). 1993;105:337-46

Mössinger HJ (see Dietrich and Mössinger). 1993;105:758-9

- (see Dietrich et al). 1993;105:712-20

Mouas C (see Menasché et al). 1993;105:353-63

Mueller JD (see Daly et al). 1993;105:904-11

Munsch C (see Rosenfeldt and Munsch). 1993;105:1118-9 (Letter reply)

Murakami T (see Kohno et al). 1993;105:729-31

Murtaugh P (see Tazelaar et al). 1993;105:674-8

Müslümanoğlu M (see İğci et al). 1993;105:1116-8 (Letter)

Muster AJ, Mavroudis C. Invited letter concerning: the importance of pulsatile flow when systemic venous return is connected directly to the pulmonary arteries. 1993;105:175-6 (Letter reply)
_, Zales VR, Ilbawi MN, Backer CL, Duffy CE, Mavroudis C. Biventricular repair of hypoplastic right ventricle assisted by pulsatile bidirectional cavopulmonary anastomosis. 1993;105:112-9

Myers JL. Discussion of Bove et al. 1993; 105:289-96

-Waldhausen JA, Cyran SE, Gleason MM, Weber HS, Baylen BG. Results of surgical repair of congenital supravalvular aortic stenosis. 1993; 105:281-8

Mylet DM (see Hartman et al). 1993;105:743-8

\section{N}

Nadel HR (see Wemer et al). 1993;105:737-42

Nagatsu M (see Yamagishi et al). 1993;105:1067-76

Naik S (see Finn et al). 1993;105:234-41

Nance ML (see Llaneras et al). 1993;105:439-43

Nanda NC (see Hanley et al). 1993;105:406-27

Neirotti R (see Akiba et al). 1993;105:142-6

Nelson RM Jr (see Leuschen et al). 1993;105:885-91

Nessly M (see Tobe et al). 1993;105:1007-14

Niczyporuk MA (see Castro et al). 1993;105:643-59

Nilsson FN (see Tazelaar et al). 1993;105:674-8

Ning X-H, Ding X, Childs KF, Bolling SF, Gallagher KP. Flavone improves functional recovery after ischemia in isolated reperfused rabbit hearts. 1993; 105:541-9

Nishigaki K (see Kawashima et al). 1993;105:591-7

Nitta S-i (see Sato et al). 1993;105:313-20

Noirclerc M (see Massard et al). 1993;105:9-14

Noirhomme P (see Laborde et al). 1993;105:278-80

Noishiki Y, Tomizawa Y, Yamane Y, Okoshi T, Satoh S, Matsumoto A. Acceleration of neointima formation in vascular prostheses by transplantation of autologous venous tissue fragments: application to small-diameter grafts. 1993; 105:796-804

Nojima T (see Tabata et al). 1993;105:598-604

Nomura F (see Aoki et al). 1993;105:207-13

Novoa R (see Lytle et al). 1993;105:605-14

Noyez L, van Son JAM, van der Werf T, Knape JTA, Gimbrère J, van Asten WNJC, Lacquet LK, Flameng W. Retrograde versus antegrade delivery of cardioplegic solution in myocardial revascularization: a clinical trial in patients with three-vessel coronary artery disease who underwent myocardial revascularization with extensive use of the internal mammary artery. 1993;105:854-63

Obo H (see Date et al). 1993;105:480-91

Oda T, Miyamoto A-T, Okamoto Y, Ban T. Skeletal muscle-powered ventricle: effects of size and configuration on ventricular function. 1993; 105:68-77

Oga K (see Yaku et al). 1993; 105:679-88

Ohgoshi Y (see Yaku et al). 1993;105:679-88

Ohishi K (see Isomura et al). 1993;105:1088-94

Ohmi M (see Sato et al). 1993;105:313-20

Ohta M (see Ichinose et al). 1993;105:1041-6

Oka T (see Yaku et al). 1993; 105:679-88

Okamoto Y (see Oda et al). 1993;105:68-77

Okoshi T, Soldani G, Goddard M, Galletti PM. Very small-diameter polyurethane vascular prostheses with rapid endothelialization for coronary artery bypass grafting. 1993;105:791-5 - (see Noishiki et al). 1993;105:796-804

O'Neill MJ Jr (see Palanzo and O'Neill). 1993; 105:1118 (Letter)

Ongsiek B (see Inui et al). 1993;105:474-9

Onizuka M, Miyauchi T, Mitsui K, Suzuki N, Ueno H, Goto K, Masaki T, Hori M. Plasma levels of endothelin-1 and thrombinantithrombin III complex in patients undergoing open chest operations. 1993;105:559-60 (Letter)

Onnasch DGW (see Sievers et al). 1993;105:580-90

Once M (see Tabata et al). 1993;105:598-604

Oostuizen JG (see Knott-Craig et al). 1993;105:394-7 
Opie LH (see Owen et al). 1993;105:995-1006

Orringer MB, Marshall B, Stirling MC. Transhiatal esophagectomy for benign and malignant disease. 1993;105:265-77

Orszulak TA, Schaff HV, DeSmet J-M, Danielson GK, Pluth JR, Puga FJ. Late results of valve replacement with the Björk-Shiley valve (1973 to 1982). 1993;105:302-12

Ottino G (see Rabajoli et al). 1993;105:948-9 (Letter)

Oury JH. Discussion of Llaneras et al. 1993;105:439-43

Øvrum E, Åm Holen E, Abdelnoor M, Øystese R, Ringdal ML. Tranexamic acid (Cyklokapron) is not necessary to reduce blood loss after coronary artery bypass operations. 1993;105:78-83

Owen P, du Toit EF, Opie LH. The optimal glucose concentration for intermittent cardioplegia in isolated rat heart when added to St. Thomas' Hospital cardioplegic solution. 1993;105:995-1006

Øystese R (see Øvrum et al). 1993;105:78-83

\section{p}

Pae WE (see Pierce et al). 1993;105:520-4

Pairolero PC (see Allen et al). 1993;105:253-9

Palanzo DA, O'Neill MJ Jr. Particle-induced coronary vasoconstriction: the need for in-line filtration of cardioplegic solutions. 1993; 105:1118 (Letter)

Palcic B (see Lam et al). 1993; 105:1035-40

Panday S (see Arsiwala and Panday). 1993;105:952-3 (Letter)

Papa M (see Como et al). 1993;105:369-71 (Letter)

Parish MA (see Galloway et al). 1993;105:781-90

Parker D (see Butler et al). 1993; 105:25-30

Parquin FJF (see Dartevelle et al). 1993;105:1025-34

Pasic M, Carrel T, von Segesser L, Turina M. In situ repair of mycotic aneurysm of the ascending aorta. 1993;105:321-6

Pasquini L (see Di Donato et al). 1993;105:398-405

Patterson GA. Discussion of Dartevelle et al. 1993;105:1025-34

- Discussion of Miller and DeHoyos. 1993;105:247-52

. Discussion of Rusch et al. 1993;105:97-106

(see Hirai et al). 1993;105:1-8

Paull DL (see Anderson et al). 1993; 105:444-52

Pavie A (see Jault et al). 1993;105:1106-13

Pearl JM, Laks H, Drinkwater DC, Meneshian A, Sun B, Gates RN, Chang P. Normocalcemic blood or crystalloid cardioplegia provides better neonatal myocardial protection than does low-calcium cardioplegia. 1993;105:201-6

- (see Gates et al). 1993;105:845-53

Pearson FG. Discussion of Orringer et al. 1993;105:265-77

Petry A (see Sievers et al). 1993;105:775-80

Peynet J (see Menasché et al). 1993;105:353-63

Pierce WS, Snyder AJ, Rosenberg G, Weiss WJ, Pae WE, Waldhausen JA. A long-term ventricular assist system. 1993; 105:520-4

Pillai R (see Butler et al). 1993; 105:25-30

Pinkard J, Utley JR, Leyland SA, Morgan M, Johnson H. Relative risk of aortic and femoral insertion of intraaortic balloon pump after coronary artery bypass grafting procedures. 1993;105:721-8

Pirie GE (see Werner et al). 1993;105:737-42

Piwnica A (see Menasché et al). 1993;105:353-63

Planché C, Serraf A, Comas JV, Lacour-Gayet F, Bruniaux J, Touchot A. Anatomic repair of transposition of great arteries with ventricular septal defect and aortic arch obstruction: one-stage versus two-stage procedure. 1993;105:925-33

Plötz FB, van Oeveren W, Bartlett RH, Wildevuur CRH. Blood activation during neonatal extracorporeal life support. 1993;105:823-32

Pluth JR (see Orszulak et al). 1993;105:302-12

Pomerantz M. Discussion of Grégoire et al. 1993;105:918-24

Popoff G (see Allen et al). 1993; 105:864-84

Pradier F (see Menasché et al). 1993;105:353-63

Presbitero P (see Rabajoli et al). 1993;105:948-9 (Letter)

Pridjian AK (see Bove et al). 1993;105:289-96

- (see Bove et al). 1993; 105:1057-66
Profio AE (see Lam et al). 1993;105:1035-40

Provenier F (see De Smet et al). 1993;105:946-7 (Letter)

Puga FJ (see Orszulak et al). 1993; 105:302-12

Puskas JD (see Hirai et al). 1993;105:1-8

\section{$\mathbf{R}$}

Rabajoli F, Presbitero P, Ottino G, Zattera G. Surgical correction of a recurrent aneurysm of the ascending aorta simulating pulmonary stenosis. 1993; 105:948-9 (Letter)

Rabaud M (see Fradin et al). 1993; 105:364-5 (Letter)

Rajagopal P (see Gokhale et al). 1993;105:557 (Letter)

Rakić S, Djuranović S. Lengths of different routes for esophageal replacement in a white population. 1993;105:1122 (Letter)

Rand WM (see Daly et al). 1993;105:904-11

Rankin JS. Discussion of Hennein et al. 1993;105:624-32

Rauch AE (see Scharfman et al). 1993; 105:765-6 (Letter)

Rayhill SC (see Castro et al). 1993;105:643-59

Razook JD (see Hedrick et al). 1993;105:297-301

Razzouk AJ. Discalssion of Gates et al. 1993;105:845-53

- (see Bailey et al). 1993; 105:805-15

Reardon MJ (see Asimacopoulos et al). 1993;105:764-5 (Letter)

Rees GM (see John et al). 1993;105:816-22

Reeves RC (see Urthaler et al). 1993;105:1114-6 (Letter)

Reichart B (see Markewitz et al). 1993;105:15-24

Reid C (see Cmolik et al). 1993; 105:222-8

Reynaud M (see Massard et al). 1993;105:9-14

Riberi A (see Metras et al). 1993;105:186-8 (Letter)

Rice TW (see Rusch et al). 1993;105:97-106

Richter JA (see Dietrich et al). 1993;105:712-20

Rinaldi M (see Tazelaar et al). 1993;105:674-8

Rinelli G (see Di Donato et al). 1993;105:398-405

Ringdal ML (see Øvrum et al). 1993;105:78-83

Rivers S (see Matsuura et al). 1993;105:45-51

Rizzi A (see Rocco et al). 1993;105:944-5 (Letter)

Rizzoli G (see Scalia et al). 1993;105:633-42

Robicsek F, Harbold NB Jr, Cappelman WF, Matos-Cruz M. Aneurysm of saphenous vein graft used for aorta-coronary bypass, resembling an anterior mediastinal mass. 1993;105:949-51 (Letter) (see Hastings and Robicsek). 1993;105:165-7

Robles de Medina EO (see Bakker et al). 1993;105:327-36

Robustellini M (see Rocoo et al). 1993; 105:944-5 (Letter)

Rocco G, Robustellini M, Rossi G, Della Pona C, Rizzi A, Crasti B. Solitary bone plasmacytoma of rib presenting as a superior sulcus tumor. 1993;105:944-5 (Letter)

Rocker GM (see Butler et al). 1993; 105:25-30

Rofsky NM (see Galloway et al). 1993;105:781-90

Rohwedder EB (see Sievers et al). 1993;105:580-90

Rose E. Discussion of Cmolik et al. 1993;105:222-8

Rosen W (see Browdie et al). 1993;105:732-6

Rosenberg G (see Pierce et al). 1993;105:520-4

Rosenfeldt F, Munsch C. Particle-induced coronary vasoconstriction: the need for in-line filtration of cardioplegic solutions. 1993;105: 1118-9 (Letter reply)

Rosenthal A (see Bove et al). 1993; 105:1057-66

Ross D. Discussion of Clarke et al. 1993;105:934-42

Rossi G (see Rocco et al). 1993; 105:944-5 (Letter)

Rossouw G (see Knott-Craig et al). 1993;105:394-7

Rouleau J (see Grégoire et al). 1993;105:918-24

Rubin JW. Discussion of Wolfe et al. 1993;105:749-56

Rusch VW. Mesothelioma: an incurable, nonsurgically treatable disease. 1993; 105:943-4 (Letter reply)

- Albain KS, Crowley JJ, Rice TW, Lonchyna V, McKenna R Jr, Livingston RB, Griffin BR, Benfield JR. Surgical resection of stage IIIA and stage IIIB non-small-cell lung cancer after concurrent induction chemoradiotherapy: a Southwest Oncology Group trial. 1993;105:97-106 
- (see Burt et al). 1993;105:89-96

Ruskin JN (see Mitchell et al). 1993;105:453-63

Sade RM. Discussion of Myers et al. 1993;105:281-8

_- (see Hanley et al). 1993; 105:406-27

Sagi B (see Mohr et al). 1993;105:371-3 (Letter)

Saint Maurice O (see Laborde et al). 1993;105:278-80

Salati M, Scrofani R, Fundaró P, Cialfi A, Santoli C. Correction of anterior mitral prolapse: results of chordal transposition (1992; 104:1268-73). 1993;105:1056 (Correction)

Samaja M (see Corno and Samaja). 1993;105:373 (Letter)

Sanchez JA (see Higgins et al). 1993; 105:965-71

Sandberg N (see Gatzinsky et al). 1993;105:553-5 (Letter)

Sandberg N (see Gatzinsky et al). 1993;105:556-7 (Letter reply)

Santoro F (see Como et al). 1993; 105:369-71 (Letter)

Sapsford RN (see Bidstrup et al). 1993; 105:147-53

Saraswati V (see Gokhale et al). 1993;105:557 (Letter)

Samaik AP (see Hackbarth et al). 1993;105:31-6

Sarsam MAI, Yacoub M. Remodeling of the aortic valve anulus. 1993;105:435-8

Sato N, Miura M, Itoh T, Ohmi M, Haneda K, Mohri H, Nitta S-i, Tanaka M. Sound spectral analysis of prosthetic valvular clicks for diagnosis of thrombosed Björk-Shiley tilting standard disc valve prostheses. 1993; 105:313-20

Satoh S (see Noishiki et al). 1993;105:796-804

Sawatari K (see Kadoba et al). 1993;105:132-41

Scalia D, Rizzoli G, Campanile F, Melacini P, Villanova C, Milano A, Fasoli G, Mazzucco A, Casarotto D. Long-term results of mitral commissurotomy. 1993;105:633-42

Schäfers H-J (see Inui et al). 1993;105:474-9

Schaff HV. Discussion of Clarke et al. 1993;105:934-42

- (see Orszulak et al). 1993;105:302-12

Scharfman WB, Rauch AE, Ferraris V, Burkart PT. Treatment of a patient with factor IX deficiency (hemophilia B) with coronary bypass surgery. 1993;105:765-6 (Letter)

Schnauffer MJ (see Lytle et al). 1993; 105:605-14

Schoen FJ (see Flomenbaum and Schoen). 1993;105:154-64

— (see Sellke et al). 1993;105:52-8

Schuurman H-J, Wijngaard PLJ, Jansen EWL, van der Meulen A, Slootweg PJ, Gmelig Meyling FHJ. Cytoimmunologic monitoring for rejection and infection after lung transplantation. 1993;105: 178-80 (Letter)

—_ (see Wijngaard et al). 1993;105:183-4 (Letter)

- (see Wijngaard et al). 1993; 105:185 (Letter reply)

Sciolaro CM (see Bailey et al). 1993;105:805-15

Seifert PE (see Hazelrigg et al). 1993; 105:389-93

Seigler HF (see Wolfe et al). 1993; 105:749-56

Sellke FW, Shafique T, Schoen FJ, Weintraub RM. Impaired endothelium-dependent coronary microvascular relaxation after cold potassium cardioplegia and reperfusion. 1993;105:52-8

Sepulvada R (see Tobe et al). 1993;105:1007-14

Serraf A (see Planché et al). 1993;105:925-33

Sett SS, Hudon MPJ, Jamieson WRE, Chow AW. Prosthetic valve endocarditis: experience with porcine bioprostheses. 1993;105:428-34

Shafique T (see Sellke et al). 1993;105:52-8

Shale DJ (see Butler et al). 1993; 105:25-30

Shapiro S (see Galloway et al). 1993;105:781-90

Shemin RJ (see Matsuura et al). 1993;105:45-51

Shennib H. Discussion of Armitage et al. 1993;105:337-46

—. Discussion of Inui et al. 1993;105:474-9

- Discussion of Miller and DeHoyos. 1993;105:247-52

- (see Adoumie et al). 1993;105:229-33

Sherman MP (see Matheis et al). 1993; 105:513-9

Shimazaki Y (see Kawashima et al). 1993;105:591-7

Shinoka T (see Yamagishi et al). 1993; 105:1067-76
Shiraisi S (see Tabata et al). 1993;105:598-604

Short BL (see Martin and Short). 1993;105:373-4 (Letter reply)

Sievers H-H, Leyh R, Loose R, Guha M, Petry A, Bemhard A. Time course of dimension and function of the autologous pulmonary root in the aortic position. 1993; 105:775-80

- Storde U, Rohwedder EB, Lange PE, Onnasch DGW, Heintzen $\mathrm{PH}$, Bemhard A. Superior function of a bicuspid over a monocuspid patch for reconstruction of a hypoplastic pulmonary root in pigs. 1993;105:580-90

Silverman NA. Invited letter conceming: cardiac stun syndrome. 1993; 105:374-5 (Letter)

Simmons TW (see McCarthy et al). 1993;105:1077-87

Skinner DB. Discussion of Streitz et al. 1993;105:383-8

- (see Altorki et al). 1993;105:260-4

Slinger P (see Adoumie et al). 1993;105:229-33

Sloane JA (see Byme et al). 1993;105:689-93

Slootweg PJ (see Schuurman et al). 1993;105:178-80 (Letter)

Slutsky AS (see Hirai et al). 1993;105:1-8

Smedts F (see van Son et al). 1993;105:368-9 (Letter)

Smith CW (see Dreyer et al). 1993;105:763 (Letter)

Smith GJW (see Higgins et al). 1993;105:965-71

Smith LR (see Spahn et al). 1993; 105:694-704

Snider AR (see Bove et al). 1993; 105:289-96

- (see Bove et al). 1993; 105:1057-66

Snyder AJ (see Pierce et al). 1993;105:520-4

Soldani G (see Okoshi et al). 1993;105:791-5

Sosnowski A (see Underwood et al). 1993;105:377-8 (Letter)

Spahn DR, Smith LR, Veronee CD, McRae RL, Hu W-c, Menius AJ, Lowe JE, Leone BJ. Acute isovolemic hemodilution and blood transfusion: effects on regional function and metabolism in myocardium with compromised coronary blood flow. 1993;105: 694-704

Spannagl M (see Dietrich et al). 1993;105:712-20

Spencer FC (see Galloway et al). 1993;105:781-90

Spero JA (see Cmolik et al). 1993;105:222-8

Stark JF. Discussion of Di Donato et al. 1993;105:398-405

Starzl TE (see Armitage et al). 1993;105:337-46

- (see Amitage et al). 1993;105:464-73

Stein HJ, Hoeft S, DeMeester TR. Functional foregut abnormalities in Barrett's esophagus. 1993; 105:107-11

Stewart RW (see Lytle et al). 1993;105:605-14

Stiles QR. Discussion of Anderson et al. 1993;105:444-52

Stirling MC (see Orringer et al). 1993;105:265-77

Stone CD (see Hennein et al). 1993;105:624-32

Storde U (see Sievers et al). 1993; 105:580-90

Stowe DF (see Boban et al). 1993; 105:532-40

Streets EM (see Bidstrup et al). 1993; 105:147-53

Streicher JT (see Llaneras et al). 1993;105:439-43

Streitz JM Jr. Discussion of Altorki et al. 1993;105:260-4

- Andrews CW Jr, Ellis FH Jr. Endoscopic surveillance of Barrett's esophagus: Does it help? 1993;105:383-8

Striffeler H (see Inderbitzi et al). 1993;105:84-8

Strobel S (see Finn et al). 1993;105:234-41

Suga H (see Yaku et al). 1993; 105:679-88

Sugita T (see Tabata et al). 1993; 105:598-604

Suhasini T (see Gokhale et al). 1993; 105:557 (Letter)

Suma H, Wanibuchi Y, Terada Y, Fukuda S, Takayama T, Funuta S. The right gastroepiploic artery graft: clinical and angiographic midterm results in 200 patients. $1993 ; 105: 615-23$

Sun B (see Pearl et al). 1993;105:201-6

Sun S-C (see Byme et al). 1993;105:689-93

Sunagawa M (see Altorki et al). 1993;105:260-4

Sundaresan S (see Date et al). 1993;105:480-91

Suzuki N (see Onizuka et al). 1993;105:559-60 (Letter) 
Tabata R, Kobayashi T, Mori A, Matsuno S, Watarida S, Onoe M, Sugita T, Shiraisi S, Nojima T. A computer simulation of the plasma leakage through a vascular prosthesis made of expanded polytetrafluoroethylene. 1993;105:598-604

Takayama T (see Suma et al). 1993;105:615-23

Tanaka M (see Sato et al). 1993;105:313-20

Tatou E (see Metras et al). 1993;105:186-8 (Letter)

Taylor PC (see Lytle et al). 1993;105:605-14

Tazelaar HD, Nilsson FN, Rinaldi M, Murtaugh P, McDougall JC, McGregor CGA. The sensitivity of transbronchial biopsy for the diagnosis of acute lung rejection. 1993;105:674-8

Tchervenkov CI. Discussion of Bove et al. 1993;105:289-96

- Discussion of Planché et al. 1993;105:925-33

Terada Y (see Suma et al). 1993;105:615-23

Thomas P (see Massard et al). 1993;105:9-14

Tidwell SL (see Anderson et al). 1993;105:444-52

Tilanus HW, Hop WCJ, Langenhorst BLAM, van Lanschot JJB. Esophagectomy with or without thoracotomy: Is there any difference? 1993;105:898-903

Tixier DB (see Matheis et al). 1993;105:513-9

Tobe CE, Vocelka C, Sepulvada R, Gillis B, Nessly M, Verrier ED, Hofer BO. Infusion of autologous platelet rich plasma does not reduce blood loss and product use after coronary artery bypass: a prospective, randomized, blinded study. 1993;105:1007-14

Todd D (see Browdie et al). 1993;105:732-6

Tokunaga K (see Mayumi and Tokunaga). 1993;105:1 120-1 (Letter)

Tomizawa Y (see Noishiki et al). 1993;105:796-804

Tönz M, von Segesser LK, Turina M. Selective pulmonary vasodilatation with inhaled nitric oxide. 1993; 105:760-2 (Letter)

Torchiana DF (see Mitchell et al). 1993;105:453-63

Touchot A (see Planché et al). 1993;105:925-33

Trastek VF. Discussion of Streitz et al. 1993; 105:383-8

_- (see Allen et al). 1993;105:253-9

Treanor P (see Matsuura et al). 1993; 105:45-51

Trohman RG (see McCarthy et al). 1993;105:1077-87

Tsao P (see Caspi et al). 1993:105:59-67

Tumiati L (see Yau et al). 1993; 105:833-44

Tuntelder JR (see Bakker et al). 1993;105:327-36

Turina M (see Pasic et al). 1993;105:321-6

- (see Tönz et al). 1993;105:760-2 (Letter)

Tyers GFO. Discussion of Aoki et al. 1993;105:207-13

_. Discussion of Cmolik et al. 1993;105:222-8

\section{U}

Uemura H (see Kawashima et al). 1993;105:591-7

Ueno H (see Onizuka et al). 1993;105:559-60 (Letter)

Ukoha O (see Burt et al). 1993; 105:89-96

Underwood MJ, Sosnowski A, Graham TR, Firmin RK. Operative management of the calcified aorta: a practical method of aortic $O c$ clusion. 1993; 105:377-8 (Letter)

Underwood SM (see Davies et al). 1993;105:979-87

Underwood SR (see Bidstrup et al). 1993;105:147-53

University of Toronto and Washington University Lung Transplant Programs (see Miller et al). 1993;105:247-52

Unruh H. Discussion of Higgins et al. 1993; 105:965-71

Urthaler F, Walker AA, Reeves RC, Hefner LL. Effects of hypoxia on intracellular calcium and contractility. 1993;105:1114-6 (Letter)

Utley JR (see Pinkard et al). 1993;105:721-8

\section{V}

van Asten WNJC (see Noyez et al). 1993;105:854-63 van Beyeren I (see Bakker et al). 1993;105:327-36

van der Meulen A (see Schuurman et al). 1993;105:178-80 (Letter)

van der Tweel I (see Bakker et al). 1993;105:327-36 van der Werf T (see Noyez et al). 1993;105:854-63

van de Wal HJCM. Total anomalous pulmonary venous drainage by double connection corrected by ascending vein and coronary sinus repair. 1993;105:367-8 (Letter)

van Lanschot JJB (see Tilanus et al). 1993;105:898-903

van Oeveren W (see Plötz et al). 1993; 105:823-32

van Son JAM, Lacquet LK, Smedts F. Pattems of ductal tissue in coarctation of the aorta in early infancy. 1993;105:368-9 (Letter)

- (see Noyez et al). 1993;105:854-63

Vargas F. Discussion of Armitage et al. 1993;105:464-73

Vaughn AL (see Wolfe et al). 1993;105:749-56

Vaughn CC. Discussion of Yau et al. 1993;105:833-44

Vermeulen FEE (see Bakker et al). 1993;105:327-36

Veronee CD (see Spahn et al). 1993; 105:694-704

Verrier ED (see Tobe et al). 1993;105:1007-14

Villamor J (see García-Talavera et al). 1993; 105:767 (Letter)

Villanova C (see Scalia et al). 1993;105:633-42

Villela de Andrade Vicente $W$. A simple and versatile sling stemal retractor for intemal mammary artery harvesting. 1993;105:560-1 (Letter)

Vlahakes GJ (see Mitchell et al). 1993;105:453-63

Vocelka C (see Tobe et al). 1993;105:1007-14

von Oppell UO. Proton gradient during cardiac arrest: oxygenation of St. Thomas' Hospital cardioplegic solution and carbon dioxide level. 1993; 105:552-3 (Letter reply)

von Segesser LK (see Pasic et al). 1993;105:321-6

— (see Tönz et al). 1993;105:760-2 (Letter)

\section{w}

Wada H (see Hirai et al). 1993;105:1-8

Waddell TK (see Hirai et al). 1993;105:1-8

Wakabayashi A. Discussion of Hazelrigg et al. 1993;105:389-93 - (see Kohno et al). 1993;105:729-31

Waldhausen JA. Nina S. Braunwald (1928-1992). 1993;105:773-4 (Obit.)

- (see Myers et al). 1993;105:281-8

- (see Pierce et al). 1993;105:520-4

Walesby RK (see Davies et al). 1993;105:979-87

Walker AA (see Urthaler et al). 1993;105:1114-6 (Letter)

Walker R (see Aronson et al). 1993;105:214-21

Wallace RB (see Abd-Elfattah et al). 1993;105:1095-105

Wang N (see Bailey et al). 1993; 105:805-15

Wanibuchi Y (see Suma et al). 1993;105:615-23

Watarida S (see Tabata et al). 1993;105:598-604

Webb WR. Discussion of Hartman et al. 1993;105:743-8

Weber HS (see Myers et al). 1993;105:281-8

Weeke J (see Gøtzsche and Weeke). 1993;105:176-8 (Letter)

Wege N (see Boldt et al). 1993;105:705-11

Weinreb JC (see Galloway et al). 1993;105:781-90

Weintraub RM (see Sellke et al). 1993;105:52-8

Weisel RD (see Yau et al). 1993;105:833-44

Weiss WJ (see Pierce et al). 1993;105:520-4

Welsh P (see Berri et al). 1993;105:951 (Letter)

Wendt $P$ (see Dietrich et al). 1993;105:712-20

Wemer HA, Pirie GE, Nadel HR, Fleisher AG, LeBlanc JG. Lung volumes, mechanics, and perfusion after pulmonary resection in infancy. 1993; 105:737-42

Wemen F (see Metras et al). 1993;105:186-8 (Letter)

Wemly JA. Discussion of Tobe et al. 1993;105:1007-14

Wessel DL (see Hanley et al). 1993;105:1047-56

Westaby S (see Butler et al). 1993;105:25-30

White RD (see McCarthy et al). 1993;105:1077-87

Wickens DG (see Davies et al). 1993;105:979-87

Wiencek JG (see Aronson et al). 1993;105:214-21 
Wijngaard PLJ, Gmelig Meyling FHJ, Schuurman H-J. Invited letter conceming: transplantation tolerance and transplantation. 1993; 105:185 (Letter reply)

- - Schuurman H-J, Gmelig Meyling FHJ, Jambroes G, Borleffs JCC. Breaking of transplantation tolerance after reduction of immunosuppression. 1993;105:183-4 (Letter) - (see Schuurman et al). 1993;105:178-80 (Letter)

Wildevuur CRH (see Plö̈z et al). 1993;105:823-32

Willett LD (see Leuschen et al). 1993;105:885-91

Williams WG. Discussion of Bailey et al. 1993;105:805-15 . Discussion of Di Donato et al. 1993;105:398-405 . Discussion of Hanley et al. 1993;105:406-27

Wilson GJ (see Caspi et al). 1993;105:59-67 (see Caspi et al). 1993;105:525-31

Wolfe WG. Discussion of Galloway et al. 1993;105:781-90

- Vaughn AL, Seigler HF, Hathom JW, Leopold KA, Duhaylongsod FG. Survival of patients with carcinoma of the esophagus treated with combined-modality therapy. 1993;105: 749-56

\section{$\mathbf{Y}$}

Yacoub M (see Sarsam and Yacoub). 1993;105:435-8

Yagawa K (see Ichinose et al). 1993;105:1041-6

Yagihara T (see Kawashima et al). 1993;105:591-7

Yaku H, Goto Y, Ohgoshi Y, Kawaguchi O, Oga K, Oka T, Suga H. Determinants of myocardial oxygen consumption in fibrillating dog hearts: comparison between normothermia and hypothermia. 1993;105:679-88

Yamagishi M, Imai Y, Hoshino S, Ishihara K, Koh Y, Nagatsu M, Shinoka T, Koide M. Anatomic correction of atrioventricular discordance. 1993; 105:1067-76
Yamamoto F (see Ichikawa and Yamamoto). 1993;105:551 -2 (Letter) - (see Kawashima et al). 1993;105:591-7

Yamamoto H, Avkiran M. Left ventricular pressure overload during postnatal development: effects on coronary vasodilator reserve and tolerance to hypothermic global ischemia. 1993;105:120-31

Yamane Y (see Noishiki et al). 1993;105:796-804

Yang X (see Matsuura et al). 1993;105:45-51

Yankah C. Discussion of Abd-Elfattah et al. 1993;105:1095-105

Yano T (see Ichinose et al). 1993;105:1041-6

Yau TM, Ikonomidis JS, Weisel RD, Mickle DAG, Ivanov J, Mohabeer MK, Tumiati L, Carson S, Liu P. Ventricular function after normothermic versus hypothermic cardioplegia. 1993;105: 833-44

Yokoyama T, Derrick MJ, Lee AW. Cardiac operation with associated pulmonary resection. 1993;105:912-7

Young HH (see Matheis et al). 1993;105:513-9

Yousem SA (see Armitage et al). 1993;105:337-46

\section{2}

Zach TL (see Leuschen et al). 1993;105:885-91

Zales VR (see Muster et al). 1993;105:112-9

Zaragoza AM (see Gates et al). 1993;105:845-53

Zaroff JG (see Aronson et al). 1993; 105:214-21

Zattera G (see Rabajoli et al). 1993;105:948-9 (Letter)

Zelano JA (see Ko et al). 1993;105:1015-24

Zickmann B (see Boldt et al). 1993; 105:705-11

Ziemer G. Discussion of Bove et al. 1993;105:1057-66

_. Discussion of Di Donato et al. 1993;105:398-405

. Discussion of Matheis et al. 1993;105:513-9

Zoia E (see Como et al). 1993;105:369-71 (Letter) 


\section{Subject index*}

A

Abscess

Prosthetic valve endocarditis with ring abscesses: surgical management and long-term results (Jault et al). 1993; 105:1106-13

Actuarial analysis

Actuarial five-year survival estimates (Mengoli) (Letter); (Dartevelle and Macchiarini) (Reply). 1993;105:375-7

\section{Adenocarcinoma}

Endoscopic surveillance of Barrett's esophagus: Does it help? (Streitz et al). 1993;105:383-8

Adenomatoid malformation of lung, congenital; see Cystic adenomatoid malformation of lung, congenital

Adenosine

Should adenosine continue to be ignored as a cardioprotective agent in cardiac operations? (Galiñanes et al). 1993;105:180-3 (Letter)

Adenosine deaminase

Inhibition of adenosine deaminase and nucleoside transport: utility in a model of homograft cardiac valve preimplantation processing (Abd-Elfattah et al). 1993;105:1095-105

Adjuvants, immunologic

Successful restoration of cell-mediated immune response after cardiopulmonary bypass by immunomodulation (Markewitz et al). 1993;105:15-24

Administration, inhalation

Intraoperative use of inhaled low-dose nitric oxide (Miller et al). 1993;105:550-1 (Letter)

Selective pulmonary vasodilatation with inhaled nitric oxide (Tönz et al). 1993; 105:760-2 (Letter)

Adolescence

Pediatric lung transplantation: the years 1985 to 1992 and the clinical trial of FK 506 (Armitage et al). 1993;105:337-46

Adrenal cortex hormones

Bronchial circulation after experimental lung transplantation: the effect of long-term administration of prednisolone (Inui et al). 1993;105:474-9

An evaluation of the role of omentopexy and of early perioperative corticosteroid administration in clinical lung transplantation (Miller et al). 1993;105:247-52

Agglutination

Cold agglutinins and warm heart surgery (Gokhale et al). 1993; 105:557 (Letter)

\section{Airway resistance}

Differential lung ventilation: applications beyond the operating room (Adoumie et al). 1993;105:229-33

*January, 1-192: February, 193-382: March, 383-578: April, 579-772: May, 773964: June, 965-1160.
Allograft; see Transplantation, homologous

Alprostadil

Improved ultrastructural lung preservation with prostaglandin $E_{1}$ as donor pretreatment in a primate model of heart-lung transplantation (Higgins et al). 1993;105:965-71

Prostaglandin $E_{1}$ for patients who have both heart and lung failure after cardiotomy (Mayumi and Tokunaga). 1993;105:1120-1 (Letter)

Alvedar oxygen concentration; see Blood gas analysis

American Association for Thoracic Surgery

Editorial search. 1993;105:190

Graham Traveling Fellowship. 1993;105:572-3, 768, 954, 1123

Notice of annual meeting. 1993;105: 189-90, 379, 576, 770, 956$7,1125-6$

Program for annual meeting. 1993;105:563-71

Robert E. Gross Research Fellowship. 1993;105:574-5, 769, 955, 1124

American Board of Thoracic Surgery

Notices of examination and recertification process. 1993;105:192, $381,587,772,959,1128$

Amrinone

The effects of amrinone versus dobutamine on myocardial mechanics and energetics after hypothermic global ischemia (Ko et al). 1993;105:1015-24

Anastomosis, surgical

Biventricular repair of hypoplastic right ventricle assisted by pulsatile bidirectional cavopulmonary anastomosis (Muster et al). 1993; 105:112-9

An evaluation of the role of omentopexy and of early perioperative corticosteroid administration in clinical lung transplantation (Miller et al). 1993; 105:247-52

\section{Aneurysm}

Aneurysm of saphenous vein graft used for aorta-coronary bypass, resembling an anterior mediastinal mass (Robicsek et al). 1993;105:949-51 (Letter)

Aneurysm of the membranous ventricular septum in transposition of the great arteries (Corno et al). 1993;105:369-71 (Letter)

Aneurysm, dissecting

Surgical repair of type A aortic dissection by the circulatory arrest-graft inclusion technique in sixty-six patients (Galloway et al). 1993;105:781-90

Transesophageal echography as an emergency diagnostic tool for acute aortic dissection (Le Smet et al). 1993; 105:946-7 (Letter)

Aneurysm, infected

In situ repair of mycotic aneurysm of the ascending aorta (Pasic et al). 1993;105:321-6

Angina pectoris

Selection of patients for same-day coronary bypass operations (Anderson et al). 1993;105:444-52 


\section{Angiography}

Dilating effects of isosorbide dinitrate on diameter of internal thoracic artery graft (Koike and Kimura). 1993;105:1121-2 (Letter)

Mechanical durability of pulmonary allograft conduits at systemic pressure: angiographic and histologic study in lambs (Kadoba et al). 1993;105:132-41

The right gastroepiploic artery graft: clinical and angiographic midterm results in 200 patients (Suma et al). 1993; 105:615-23

Angioplasty

Aortic aneurysm after subclavian arterial flap angioplasty for $\infty$ arctation of the aorta (Berri et al). 1993; 105:951 (Letter)

Angioplasty, transluminal, percutaneous coronary

Superiority of controlled surgical reperfusion versus percutaneous transluminal coronary angioplasty in acute coronary occlusion (Allen et al). 1993;105:864-84

Animal disease models; see Disease models, animal

Annuloplasty

Annuloplasty with flexible or rigid ring does not alter left ventricular systolic performance, energetics, or ventricular-arterial coupling in conscious, closed-chest dogs (Castro et al). 1993; 105:643-59

Anoxemia

Cardiopulmonary dysfunction produced by reoxygenation of immature hypoxemic animals supported by cardiopulmonary bypass: prevention by intravenous metabolic pretreatment (Matheis et al). 1993;105:513-9

Anoxia

Effects of hypoxia on intracellular calcium and contractility (Urthaler et al). 1993;105:1114-6 (Letter)

Antegrade cardioplegia; see Heart arrest, induced

Anticoagulants

Clinical experience with the Omnicarbon prosthetic heart valve (Misawa et al). 1993; 105:168-72

Antitachycardia pacing; see Cardiac pacing, artificial

Antithrombin III

Plasma levels of endothelin-1 and thrombin-antithrombin III complex in patients undergoing open chest operations (Onizuka Aorta et al). 1993;105:559-60 (Letter)

In situ repair of mycotic aneurysm of the ascending aorta (Pasic et al). 1993;105:321-6

Operative management of the calcified aorta: a practical method of aortic occlusion (Underwood et al). 1993; 105:377-8 (Letter)

Relative risk of aortic and femoral insertion of intraaortic balloon pump after coronary artery bypass grafting procedures (Pinkard et al). 1993; 105:721-8

Surgical correction of a recurrent aneurysm of the ascending aorta simulating pulmonary stenosis (Rabajoli et al). 1993;105:9489 (Letter)

Transposition of the great arteries with posterior aorta (Antunes). 1993;105:369 (Letter)

\section{Aorta, abdominal}

Thoracic wall necrosis in a patient with intemal mammary-coronary bypass after prosthetic replacement of the thoracoabdominal aorta (Aebert et al). 1993;105:557-8 (Letter)

Aorta, thoracic

Anatomic repair of transposition of great arteries with ventricular septal defect and aortic arch obstruction: one-stage versus twostage procedure (Planché et al). 1993;105:925-33

The management of severe subaortic stenosis, ventricular septal defect, and aortic arch obstruction in the neonate (Bove et al). 1993; 105:289-96

Tetralogy of Fallot with pulmonary atresia, coronary artery-pulmonary artery fistula, and origin of left pulmonary artery from descending aorta: total correction in infancy (Metras et al). 1993; 105:186-8 (Letter)
Thoracic wall necrosis in a patient with intemal mammary-coronary bypass after prosthetic replacement of the thoracoabdominal aorta (Aebert et al). 1993;105:557-8 (Letter)

A variant course of posterior descending artery (Arsiwala and Panday). 1993;105:952-3 (Letter)

\section{Aortic aneurysm}

Aortic aneurysm after subclavian arterial flap angioplasty for $c^{-}$ arctation of the aorta (Berri et al). 1993; 105:951 (Letter)

Surgical correction of a recurrent aneurysm of the ascending aorta simulating pulmonary stenosis (Rabajoli et al). 1993;105:9489 (Letter)

Transesophageal echography as an emergency diagnostic tool for acute aortic dissection (De Smet et al). 1993;105:946-7 (Letter)

Aortic arch; see Aorta, thoracic

Aortic coarctation

Aortic aneurysm after subclavian arterial flap angioplasty for coarctation of the aorta (Berri et al). 1993;105:951 (Letter)

Patterns of ductal tissue in coarctation of the aorta in early infancy (van Son et al). 1993;105:368-9 (Letter)

Aortic dissection; see Aneurysm, dissecting

Aortic valve

Degeneration of aortic valve allografts in young recipients (Clarke et al). 1993;105:934-42

Effects of fixation back pressure and antimineralization treatment on the morphology of porcine aortic bioprosthetic valves (Flomenbaum and Schoen). 1993;105:154-64

Time course of dimension and function of the autologous pulmonary root in the aortic position (Sievers et al). 1993;105:775-80

Aortic valve insufficiency

Remodeling of the aortic valve anulus (Sarsam and Yacoub). 1993; 105:435-8

Aortic valve stenosis

Invited letter conceming: critical aortic stenosis (McKay) (Letter); (Freedom) (Reply). 1993; 105:365-7

The management of severe subaortic stenosis, ventricular septal defect, and aortic arch obstruction in the neonate (Bove et al) 1993; 105:289-96

Results of surgical repair of congenital supravalvular aortic stenosis (Myers et al). 1993; 105:281-8

Aortocoronary bypass; see Coronary artery bypass

Aprotinin

Comparison of two aprotinin dosage regimens in pediatric patients having cardiac operations: influence on platelet function and blood loss (Boldt et al). 1993;105:705-11

Effect of aprotinin (Trasylol) on aorta-coronary bypass graft patency (Bidstrup et al). 1993;105:147-53

Hemostatic activation during cardiopulmonary bypass with different aprotinin dosages in pediatric patients having cardiac operations (Dietrich et al). 1993;105:712-20

Invited letter conceming: aprotinin use in pediatric cardiac operations (Edmunds) (Letter); (Dietrich and Mössinger) (Reply); (Boldt) (Reply). 1993; 105:757-60

Arrhythmia

Initial experience with the maze procedure for atrial fibrillation (McCarthy et al). 1993; 105:1077-87

Arterial occlusive diseases

The management of severe subaortic stenosis, ventricular septal defect, and aortic arch obstruction in the neonate (Bove et al). 1993; 105:289-96

Operative management of the calcified aorta: a practical method of aortic occlusion (Underwood et al). 1993;105:377-8 (Letter)

Recovery of postischemic contractile function is depressed by antegrade warm continuous blood cardioplegia (Misare et al). $1993 ; 105: 37-44$ 


\section{Arterial switch operation; see Transposition of great vessels Arteries}

Clinical evaluation with exercise performance in twenty patients who underwent coronary artery bypass grafting with both the gastroepiploic and intemal thoracic arteries (Isomura et al). 1993;105:1088-94

The right gastroepiploic artery graft: clinical and angiographic midterm results in 200 patients (Suma et al). 1993; 105:615-23

\section{Arterio-arterial fistula}

Tetralogy of Fallot with pulmonary atresia, coronary arterypulmonary artery fistula, and origin of left pulmonary artery from descending aorta: total correction in infancy (Metras et al). 1993;105:186-8 (Letter)

Ascending aorta; see Aorta

Atrial fibrillation

Initial experience with the maze procedure for atrial fibrillation (McCarthy et al). 1993; 105:1077-87

Atrioventricular discordance; see Heart defects, congenital

Autologous blood transfusion; see Blood transfusion, autologous

B

Baby; see Infant

Balloon dilatation

Long-term results of mitral commissurotomy (Scalia et al). 1993; 105:633-42

Barrett esophagus

Endoscopic surveillance of Barrett's esophagus: Does it help? (Streitz et al). 1993;105:383-8

Functional foregut abnormalities in Barrett's esophagus (Stein et al). 1993;105:107-11

Bicuspld patch; see Bioprosthesis

Biocompatibie materials

Preliminary experimental results of a new resorbable biomaterial as pericardial substitute (Fradin et al). 1993;105:364-5 (Letter)

Biological transport

Inhibition of adenosine deaminase and nucleoside transport: utility in a model of homograft cardiac valve preimplantation processing (Abd-Elfattah et al). 1993;105:1095-105

Bioprosthesis

Effects of fixation back pressure and antimineralization treatment on the morphology of porcine aortic bioprosthetic valves (Flomenbaum and Schoen). 1993; 105:154-64

Prosthetic valve endocarditis: experience with porcine bioprostheses (Sett et al). 1993;105:428-34

Superior function of a bicuspid over a monocuspid patch for reconstruction of a hypoplastic pulmonary root in pigs (Sievers et al). 1993;105:580-90

Biopsy

The sensitivity of transbronchial biopsy for the diagnosis of acute lung rejection (Tazelaar et al). 1993;105:674-8

Björk-Shiley valve; see Heart valve prosthesis

Bleeding; see Hemorrhage

Bleomycins

Comparison of insufflated talc under thoracoscopic guidance with standard tetracycline and bleomycin pleurodesis for control of Blood malignant pleural effusions (Hartman et al). 1993;105:743-8

The hemostatic effect of autologous platelet-rich plasma versus autologous whole blood after cardiac operations: Is platelet separation really necessary? (Mohr et al). 1993;105:371-3 (Letter)
Blood activation; see Platelet activation

Blood cardioplegia; see Heart arrest, induced

Blood circulation

Bronchial circulation after experimental lung transplantation: the effect of long-term administration of prednisolone (Inui et al). 1993; 105:474-9

Time course of dimension and function of the autologous pulmonary root in the aortic position (Sievers et al). 1993;105:77580

Blood component transfusion

Infusion of autologous platelet rich plasma does not reduce blood loss and product use after coronary artery bypass: a prospective, randomized, blinded study (Tobe et al). 1993;105:100714

Blood gas analysis

Changes in alveolar oxygen and carbon dioxide concentration and oxygen consumption during lung preservation: the maintenance of aerobic metabolism during lung preservation (Date et al). 1993;105:492-501

Blood loss; see Hemorrhage

Blood platelets

The hemostatic effect of autologous platelet-rich plasma versus autologous whole blood after cardiac operations: Is platelet separation really necessary? (Mohr et al). 1993;105:371-3 (Letter)

Infusion of autologous platelet rich plasma does not reduce blood loss and product use after coronary artery bypass: a prospective, randomized, blinded study (Tobe et al). 1993;105:1007-14

Inhibition of platelet function by heparin: an etiologic factor in postbypass hemorihage (John et al). 1993;105:816-22

Blood transfusion

Acute isovolemic hemodilution and blood transfusion: effects on regional function and metabolism in myocardium with compromised coronary blood flow (Spahn et al). 1993;105:694-704

Blood transfusion, autologous

The hemostatic effect of autologous platelet-rich plasma versus autologous whole blood after cardiac operations: Is platelet separation really necessary? (Mohr et al). 1993;105:371-3 (Letter)

Blood vessel prosthesis

Acceleration of neointima formation in vascular prostheses by transplantation of autologous venous tissue fragments: application to small-diameter grafts (Noishiki et al). 1993;105: 796-804

A computer simulation of the plasma leakage through a vascular prosthesis made of expanded polytetrafluoroethylene (Tabata et al). 1993;105:598-604

Remodeling of the aortic valve anulus (Sarsam and Yacoub). 1993; $105: 435-8$

Thoracic wall necrosis in a patient with intemal mammary-coronary bypass after prosthetic replacement of the thoracoabdominal aorta (Aebert et al). 1993;105:557-8 (Letter)

Very small-diameter polyurethane vascular prostheses with rapid endothelialization for coronary artery bypass grafting (Okoshi et al). 1993;105:791-5

Blood vessels

Acceleration of neointima formation in vascular prostheses by transplantation of autologous venous tissue fragments: application to small-diameter grafts (Noishiki et al). 1993;105: 796-804

Allograft replacement of the trachea: experimental synchronous revascularization of composite thyrotracheal transplant (Khalil-Marzouk). 1993;105:242-6

The microvascular distribution of cardioplegic solution in the piglet heart: retrograde versus antegrade delivery (Gates et al). 1993; 105:845-53 
Blunt injury; see Wounds, nonpenetrating

Bone neoplasms

Pleural effusion as the first manifestation of a malignant fibrous histiocytoma (García-Talavera et al). 1993;105:767 (Letter)

Solitary bone plasmacytoma of rib presenting as a superior sulcus tumor (Rocco et al). 1993; 105:944-5 (Letter)

Bovine thrombin; see Thrombin

Brachiocephalic trunk

Blunt injury of the innominate artery: use of a vascular pericardial flap (Asimacopoulos et al). 1993;105:764-5 (Letter)

Braunwald, Nina S.

Nina S. Braunwald (1928-1992) (Waldhausen). 1993;105:773-4 (Obit.)

Bronchial arteries

Management and prognosis of massive hemoptysis: recent experience with 120 patients (Knott-Craig et al). 1993;105:394-7

Bronchial circulation; see Blood circulation

Bronchogenic carcinoma; see Carcinoma, bronchogenic

Butanedione monoxime

Effects of 2,3-butanedione monoxime in isolated hearts: protection during reperfusion after global ischemia (Boban et al). 1993; 105:532-40

C

\section{Calcification}

Effects of fixation back pressure and antimineralization treatment on the morphology of porcine aortic bioprosthetic valves (Flomenbaum and Schoen). 1993;105:154-64

Operative management of the calcified aorta: a practical method of aortic occlusion (Underwood et al). 1993;105:377-8 (Letter)

Calcium

Effect of calcium and preischemic hypothermia on recovery of myocardial function after cardioplegic ischemia in neonatal lambs (Aoki et al). 1993;105:207-13

Effects of high plasma epinephrine and $\mathrm{Ca}^{2+}$ concentrations on neonatal myocardial function after ischemia (Caspi et al). 1993; 105:59-67

Effects of hypoxia on intracellular calcium and contractility (Urthaler et al). 1993;105:1114-6 (Letter)

Improved recovery of heart transplants with a specific kit of preservation solutions (Menasché et al). 1993;105:353-63

Normocalcemic blood or crystalloid cardioplegia provides better neonatal myocardial protection than does low-calcium cardioplegia (Pearl et al). 1993;105:201-6

Redistribution of myocardial calcium during ischemia: relationship to onset of contracture (Jimenez et al). 1993;105:988-94

Carbon dioxide

Proton gradient during cardiac arrest: oxygenation of St. Thomas' Hospital cardioplegic solution and carbon dioxide level (Ichikawa and Yamamoto) (Letter); (von Oppell) (Reply). 1993;105:551-3

Carbon dioxide concentration; see Blood gas analysis

Carcinoma

Detection of dysplasia and carcinoma in situ with a lung imaging fluorescence endoscope device (Lam et al). 1993;105:1035-40

Esophagectomy with or without thoracotomy: Is there any difference? (Tilanus et al). 1993; 105:898-903

Hypotonic cisplatin treatment for carcinomatous pleuritis found at thoracotomy in patients with lung cancer: in vitro experiments and preliminary clinical results (Ichinose et al). 1993;105: $1041-6$

Survival of patients with carcinoma of the esophagus treated with combined-modality therapy (Wolfe et al). 1993;105:749-56
Carcinoma, bronchogenic

Cardiac operation with associated pulmonary resection (Yokoyama et al). 1993; 105:912-7

Carcinoma, non-small cell lung

Anterior transcervical-thoracic approach for radical resection of lung tumors invading the thoracic inlet (Dartevelle et al). 1993;105:1025-34

Surgical resection of stage IIIA and stage IIIB non-small-cell lung cancer after concurrent induction chemoradiotherapy: a Southwest Oncology Group trial (Rusch et al). 1993;105:97-106

Cardiac anomalies, congenital; see Heart defects, congenital

Cardiac arrest; see Heart arrest, induced

Cardiac-derived thromboxane A2; see Thromboxane A2

Cardiac pacing, artificial

Clinical significance of epicardial pacing wire cultures (Hastings and Robicsek). 1993;105:165-7

Experience with an implantable tiered therapy device incorporating antitachycardia pacing and cardioverter/defibrillator therapy (Mitchell et al). 1993;105:453-63

Cardiac stun

Invited letter concerning: cardiac stun syndrome (Silverman). 1993; 105:374-5 (Letter)

The reoxygenation phenomenon (Como and Samaja) (Letter); (Martin and Short) (Reply). 1993;105:373-4

Cardiac transplantation; see Heart transplantation

Cardioplegia; see Heart arrest, induced

Cardioplegic solutions

A clinical trial of University of Wisconsin solution for pulmonary preservation (Hardesty et al). 1993; 105:660-6

Cold agglutinins and warm heart surgery (Gokhale et al). 1993; 105:557 (Letter)

A comparison of intracellular solutions for donor heart preservation (Hendry et al). 1993; 105:667-73

Impaired endothelium-dependent coronary microvascular relaxation after cold potassium cardioplegia and reperfusion (Sellke et al). 1993;105:52-8

The microvascular distribution of cardioplegic solution in the piglet heart: retrograde versus antegrade delivery (Gates et al). 1993; 105:845-53

Myocardial distribution of cardioplegic solution after retrograde delivery in patients undergoing cardiac surgical procedures (Aronson et al). 1993;105:214-21

Normocalcemic blood or crystalloid cardioplegia provides better neonatal myocardial protection than does low-calcium cardioplegia (Pearl et al). 1993;105:201-6

The optimal glucose concentration for intermittent cardioplegia in isolated rat heart when added to St. Thomas' Hospital cardioplegic solution (Owen et al). 1993;105:995-1006

Particle-induced coronary vasoconstriction: the need for in-line filtration of cardioplegic solutions (Palanzo and O'Neill) (Letter); (Rosenfeldt and Munsch) (Reply). 1993;105:1118-9

Proton gradient during cardiac arrest: oxygenation of St. Thomas' Hospital cardioplegic solution and carbon dioxide level (Ichikawa and Yamamoto) (Letter); (von Oppell) (Reply). 1993; 105:551-3

Reply to invited letter concerning: assessment of myocardial ultrastructure after retrograde infusion of cardioplegic solution (J Thorac Cardiovasc Surg 1992;104:1745-6) (Fischer and Bamer). 1993;105:762-3 (Letter)

Retrograde versus antegrade delivery of cardioplegic solution in myocardial revascularization: a clinical trial in patients with three-vessel coronary artery disease who underwent myocardial revascularization with extensive use of the internal mammary artery (Noyez et al). 1993;105:854-63

Ventricular function after normothermic versus hypothermic cardioplegia (Yau et al). 1993;105:833-44 


\section{Cardiopulmonary bypass}

Blood activation during neonatal extracorporeal life support (Plötz et al). 1993;105:823-32

Cardiopulmonary dysfunction produced by reoxygenation of immature hypoxemic animals supported by cardiopulmonary bypass: prevention by intravenous metabolic pretreatment (Matheis et al). 1993; 105:513-9

Effect of cardiopulmonary bypass on systemic release of neutrophil elastase and tumor necrosis factor (Butler et al). 1993; 105:25-30

Exclusion of the placenta during fetal cardiac bypass augments systemic flow and provides important information about the mechanism of placental injury (Fenton et al). 1993;105:502-12

Hemostatic activation during cardiopulmonary bypass with different aprotinin dosages in pediatric patients having cardiac operations (Dietrich et al). 1993; 105:712-20

Inhibition of platelet function by heparin: an etiologic factor in postbypass hemorrhage (John et al). 1993;105:816-22

Interleukin-8 release and neutrophil degranulation after pediatric cardiopulmonary bypass (Finn et al). 1993;105:234-41

Reply to: neutrophil activation during cardiopulmonary bypass (J Thorac Cardiovasc Surg 1992;104:1746-8) (Dreyer et al). 1993;105:763 (Letter)

Reply to invited letter concerning: changes in plasma-free thyroid hormones during cardiopulmonary bypass (J THORAC CARDIOVASC SURG 1992;104:526-7) (Gøtzsche and Weeke). 1993; 105:176-8 (Letter)

Successful restoration of cell-mediated immune response after cardiopulmonary bypass by immunomodulation (Markewitz et al). 1993;105:15-24

Time-course of free radical activity during coronary artery operations with cardiopulmonary bypass (Davies et al). 1993;105: 979-87

Warm versus cold blood cardioplegia-Is there a difference? (Matsuura et al). 1993;105:45-51

Cardiotomy; see Heart surgery

Cardioversion; see Electric countershock

Catecholamines

Effects of high plasma epinephrine and $\mathrm{Ca}^{2+}$ concentrations on neonatal myocardial function after ischemia (Caspi et al). 1993;105:59-67

The protective effect of magnesium on acute catecholamine cardiotoxicity in the neonate (Caspi et al). 1993; 105:525-31

Cavopulmonary anastomosis; see Anastomosis, surgical

Cell-mediated immune response; see Immunity, cellular

Cells, immunology

Cytoimmunologic monitoring for rejection and infection after lung transplantation (Schuurman et al). 1993; 105:178-80 (Letter)

Chemotherapy; see Drug therapy

Chest; see Thorax

Chest wall neoplasms; see Thoracic neoplasms

Child

Changes in plasma fibronectin in children after elective repair of congenital heart defects (Hackbarth et al). 1993;105:31-6

Comparison of two aprotinin dosage regimens in pediatric patients having cardiac operations: influence on platelet function and blood loss (Boldt et al). 1993;105:705-11

A decade (1982 to 1992) of pediatric cardiac transplantation and the impact of FK 506 immunosuppression (Armitage et al). 1993; $105: 464-73$

Degeneration of aortic valve allografts in young recipients (Clarke et al). 1993;105:934-42

Hemostatic activation during cardiopulmonary bypass with different aprotinin dosages in pediatric patients having cardiac operations (Dietrich et al). 1993;105:712-20
Interleukin-8 release and neutrophil degranulation after pediatric cardiopulmonary bypass (Finn et al). 1993;105:234-41

Intraventricular repair for Taussig-Bing anomaly (Kawashima et al). 1993;105:591-7

Invited letter conceming: aprotinin use in pediatric cardiac operations (Edmunds) (Letter); (Dietrich and Mössinger) (Reply); (Boldt) (Reply). 1993; 105:757-60

A new video-assisted thoracoscopic surgical technique for interruption of patent ductus arteriosus in infants and children (Laborde et al). 1993;105:278-80

Pediatric lung transplantation: the years 1985 to 1992 and the clinical trial of FK 506 (Armitage et al). 1993;105:337-46

\section{Chordae tendinese}

Left ventricular function in experimental mitral regurgitation with intact chordae tendineae (Hennein et al). 1993;105:624-32

\section{Christmas disease}

Treatment of a patient with factor IX deficiency (hemophilia B) with coronary bypass surgery (Scharfman et al). 1993;105: 765-6 (Letter)

Circulatory arrest; see Heart arrest, induced

Cisplatin

Hypotonic cisplatin treatment for carcinomatous pleuritis found at thoracotomy in patients with lung cancer: in vitro experiments and preliminary clinical results (Ichinose et al). 1993;105: $1041-6$

Coarctation of aorta; see Aortic coarctation

Cold agglutins; see Agglutination

Cold blood cardioplegia; see Heart arrest, induced

Combined modality therapy

Survival of patients with carcinoma of the esophagus treated with combined-modality therapy (Wolfe et al). 1993;105:749-56

\section{Commissurotomy}

Long-term results of mitral commissurotomy (Scalia et al). 1993; 105:633-42

Completion pneumonectomy; see Pneumonectomy

Computed tomography; see Tomography, $x$-ray computed

Computer simulation

A computer simulation of the plasma leakage through a vascular prosthesis made of expanded polytetrafluoroethylene (Tabata et al). 1993; 105:598-604

Congenital adenomatoid malformation of lung; see Cystic adenomatoid malformation of lung, congenital

Congenital heart defects; see Heart defects, congenital

Contractile function, postischemic; see Myocardial contraction

Coronary angiography

A variant course of posterior descending artery (Arsiwala and Panday). 1993;105:952-3 (Letter)

Coronary artery bypass

Aneurysm of saphenous vein graft used for aorta-coronary bypass, resembling an anterior mediastinal mass (Robicsek et al). 1993;105:949-51 (Letter)

Clinical evaluation with exercise performance in twenty patients who underwent coronary artery bypass grafting with both the gastroepiploic and intemal thoracic arteries (Isomura et al). 1993;105:1088-94

Dilating effects of isosorbide dinitrate on diameter of internal thoracic artery graft (Koike and Kimura). 1993;105:1121-2 (Letter)

Effect of aprotinin (Trasylol) on aorta-coronary bypass graft patency (Bidstrup et al). 1993;1C5:147-53

The effect of coronary reoperation on the survival of patients with stenoses in saphenous vein bypass grafts to coronary arteries (Lytle et al). 1993; 105:605-14

Infusion of autologous platelet rich plasma does not reduce blood loss and product use after coronary artery bypass: a prospective, randomized, blinded study (Tobe et al). 1993;105:1007-14 
Coronary artery bypass - cont'd

Relative risk of aortic and femoral insertion of intraaortic balloon pump after coronary artery bypass grafting procedures (Pinkard et al). 1993; 105:721-8

The right gastroepiploic artery graft: clinical and angiographic midterm results in 200 patients (Suma et al). 1993; 105:615-23

Selection of patients for same-day coronary bypass operations (Anderson et al). 1993;105:444-52

Thoracic wall necrosis in a patient with internal mammary-coronary bypass after prosthetic replacement of the thoracoabdominal aorta (Aebert et al). 1993;105:557-8 (Letter)

Time-course of free radical activity during coronary artery operations with cardiopulmonary bypass (Davies et al). 1993;105: 979-87

Tranexamic acid (Cyklokapron) is not necessary to reduce blood loss after coronary artery bypass operations (Øvrum et al). 1993;105:78-83

Treatment of a patient with factor IX deficiency (hemophilia B) with coronary bypass surgery (Scharfman et al). 1993;105: 765-6 (Letter)

Ventricular function after normothermic versus hypothermic cardioplegia (Yau et al). 1993;105:833-44

Very small-diameter polyurethane vascular prostheses with rapid endothelialization for coronary artery bypass grafting (Okoshi et al). 1993;105:791-5

Coronary artery-pulmonary artery fistula; see Arterio-arterial fistula

Coronary circulation

Acute isovolemic hemodilution and blood transfusion: effects on regional function and metabolism in myocardium with compromised coronary blood flow (Spahn et al). 1993;105: 694-704

\section{Coronary disease}

Retrograde versus antegrade delivery of cardioplegic solution in myocardial revascularization: a clinical trial in patients with three-vessel coronary artery disease who underwent myocardial revascularization with extensive use of the intemal mammary artery (Noyez et al). 1993;105:854-63

Superiority of controlled surgical reperfusion versus percutaneous transluminal coronary angioplasty in acute coronary occlusion (Allen et al). 1993;105:864-84

A variant course of posterior descending artery (Arsiwala and Panday). 1993;105:952-3 (Letter)

Coronary reoperation; see Reoperation

Coronary reperfusion; see Myocardial reperfusion

Coronary vasoconstriction; see Vasoconstriction

Coronary vessel anomalies

Total anomalous pulmonary venous drainage by double connection corrected by ascending vein and coronary sinus repair (van de Wal). 1993; 105:367-8 (Letter)

Coronary vessels

Impaired endothelium-dependent coronary microvascular relaxation after cold potassium cardioplegia and reperfusion (Sellke et al). 1993;105:52-8

Corrections

Correction of anterior mitral prolapse: results of chordal transposition (Salati et al) (1992;104:1268-73). 1993;105:1056

Effect of cardiopulmonary bypass on systemic release of neutrophil elastase and tumor necrosis factor (Butler et al) (1993; 105:25-30). 1993;105:1056

Correspondence

Letters. 1993; 105:173-88, 364-78, 550-62, 757-67, 943-53, 1114-22
Corticosterolds; see Adrenal cortex hormones

Costs and cost analysis

An economic analysis of heart-lung transplantation: costs, insurance coverage, and reimbursement (Evans et al). 1993;105: 972-8

Crawford, E.Stanley

E. Stanley Crawford (1922-1992). 1993;105:579

Cryopreservation

Degeneration of aortic valve allografts in young recipients (Clarke et al). 1993;105:934-42

Cryosurgery

Extensive cryoablation of the left ventricular posterior papillary muscle and subjacent ventricular wall: impact on mitral valve function and hemodynamics (Bakker et al). 1993;105:327-36

Crystalloid cardioplegia; see Heart arrest, induced

Culture

Clinical significance of epicardial pacing wire cultures (Hastings and Robicsek). 1993;105:165-7

Cyklokapron; see Tranexamic acid

Cystic adenomatoid malformation of lung, congenital

The use of "nonanatomic" pulmonary resection in infants with extensive congenital adenomatoid malformation of the lung (Browdie et al). 1993; 105:732-6

Cysts

Intrathoracic meningocele (Auge and Georg). 1993;105:561-2 (Letter)

Cytoimmunology; see Cells, immunology

\section{D}

Death rate; see Mortality

Defibrillation, electric; see Electric countershock

Deglutition disorders

Dysphagia lusoria (İgci et al). 1993;105:1116-8 (Letter)

Descending aorta; see Aorta, thoracic

Dextrans

Evaluation of lung metabolism during successful twenty-fourhour canine lung preservation (Date et al). 1993;105:480-91

Differential lung ventilation; see Respiration, artificial

Digestive system abnormalities

Functional foregut abnormalities in Barrett's esophagus (Stein et al). 1993;105:107-11

Disease models, animal

Improved ultrastructural lung preservation with prostaglandin $\mathrm{E}_{1}$ as donor pretreatment in a primate model of heart-lung transplantation (Higgins et al). 1993;105:965-71

Pathogenesis of ischemic mitral insufficiency (Llaneras et al).

Dobutamine $1993 ; 105: 439-43$

The effects of amrinone versus dobutamine on myocardial mechanics and energetics after hypothermic global ischemia (Ko et al). 1993;105:1015-24

Donor heart preservation; see Organ preservation

Donor size matching; see Organ weight

Dose-response relationship, drug

Comparison of two aprotinin dosage regimens in pediatric patients having cardiac operations: influence on platelet function and blood loss (Boldt et al). 1993;105:705-11

Hemostatic activation during cardiopulmonary bypass with different aprotinin dosages in pediatric patients having cardiac operations (Dietrich et al). 1993; 105:712-20

Double lung transplantation; see Lung transplantation

Double outlet right ventricle

Intraventricular repair for Taussig-Bing anomaly (Kawashima et al). 1993;105:591-7 


\section{Drug evaluation}

Should adenosine continue to be ignored as a cardioprotective agent in cardiac operations? (Galiñanes et al). 1993;105:180-3 (Letter)

Drug therapy

Surgical resection of stage IIIA and stage IIIB non-small-cell lung cancer after concurrent induction chemoradiotherapy: a Southwest Oncology Group trial (Rusch et al). 1993;105:97-106

Ductus arteriosus

Pattems of ductal tissue in coarctation of the aorta in early infancy (van Son et al). 1993;105:368-9 (Letter)

Ductus arteriosus, patent

A new video-assisted thoracoscopic surgical technique for interruption of patent ductus arteriosus in infants and children (Laborde et al). 1993; 105:278-80

Dysphagia lusoria; see Deglutition disorders

Dysplasia

Detection of dysplasia and carcinoma in situ with a lung imaging fluorescence endoscope device ( $\mathrm{Lam}$ et al). 1993;105:1035-40

\section{E}

\section{Echocardiography}

Myocardial distribution of cardioplegic solution after retrograde delivery in patients undergoing cardiac surgical procedures (Aronson et al). 1993;105:214-21

Transesophageal echography as an emergency diagnostic tool for acute aortic dissection (De Smet et al). 1993; 105:946-7 (Letter)

Economic analysis; see Costs and cost analysis

Editorial search

Editorial search. 1993;105:190

Elastase; see Pancreatopeptidase

Electric countershock

Experience with an implantable tiered therapy device incorporating antitachycardia pacing and cardioverter/defibrillator therapy (Mitchell et al). 1993;105:453-63

Endocarditis

Prosthetic valve endocarditis: experience with porcine bioprostheses (Sett et al). 1993;105:428-34

Prosthetic valve endocarditis with ring abscesses: surgical management and long-term results (Jault et al). 1993;105:1106-13

Endoscopy

Detection of dysplasia and carcinoma in situ with a lung imaging fluorescence endoscope device (Lam et al). 1993;105:1035-40

Endoscopic surveillance of Barrett's esophagus: Does it help? (Streitz et al). 1993;105:383-8

Endothelins

Plasma levels of endothelin-1 and thrombin-antithrombin III complex in patients undergoing open chest operations (Onizuka et al). 1993;105:559-60 (Letter)

Endothelium, vascular

Very small-diameter polyurethane vascular prostheses with rapid endothelialization for coronary artery bypass grafting (Okoshi et al). 1993:105:791-5

Endothelium-derived relaxing factor

Impaired endothelium-dependent coronary microvascular relaxation after cold potassium cardioplegia and reperfusion (Sellke et al). 1993;105:52-8

Epinephrine

Effects of high plasma epinephrine and $\mathrm{Ca}^{2+}$ concentrations on neonatal myocardial function after ischemia (Caspi et al). 1993; 105:59-67

Equipment and supplies

Detection of dysplasia and carcinoma in situ with a lung imaging fluorescence endoscope device (Lam et al). 1993;105:1035-40
Experience with an implantable tiered therapy device incorporating antitachycardia pacing and cardioverter/defibrillator therapy (Mitchell et al). 1993;105:453-63

A long-term ventricular assist system (Pierce et al). 1993;105:520-4

Thoracoscopic pleurectomy for treatment of complicated spontaneous pneumothorax (Inderbitzi et al). 1993;105:84-8

Esophageal achalasia, surgery

Invited letter conceming: technique for prevention of gastroesophageal reflux after transthoracic Heller's operation (Ellis) (Letter); (Gatzinsky et al) (Reply). 1993; 105:555-7

Technique for prevention of gastroesophageal reflux after transthoracic Heller's operation (Gatzinsky et al). 1993;105:553-5 (Letter)

Esophageal diverticulum

Thoracic esophageal diverticula: Why is operation necessary? (Altorki et al). 1993;105:260-4

Esophageal motility disorders

Thoracic esophageal diverticula: Why is operation necessary? (Altorki et al). 1993;105:260-4

Esophageal neoplasms

Esophagectomy with or without thoracotomy: Is there any difference? (Tilanus et al). 1993;105:898-903

Survival of patients with carcinoma of the esophagus treated with combined-modality therapy (Wolfe et al). 1993;105:749-56

Transhiatal esophagectomy for benign and malignant disease (Orringer et al). 1993;105:265-77

Esophagectomy

Esophagectomy with or without thoracotomy: Is there any difference? (Tilanus et al). 1993; 105:898-903

Lengths of different routes for esophageal replacement in a white population (Rakić and Djuranović). 1993;105:1122 (Letter)

Transhiatal esophagectomy for benign and malignant disease (Orringer et al). 1993; 105:265-77

Esophagus

Dysphagia lusoria (İğci et al). 1993;105:1116-8 (Letter)

Survival of patients with carcinoma of the esophagus treated with combined-modality therapy (Wolfe et al). 1993;105:749-56

Ewing's sarcoma; see Sarcoma, Ewing's

Exercise test

Clinical evaluation with exercise performance in twenty patients who underwent coronary artery bypass grafting with both the gastroepiploic and intemal thoracic arteries (Isomura et al). 1993;105:1088-94

Expectations

Fulfilling expectations (Fosburg). 1993;105:194-200 (Pres. address)

Experimental model; see Models, theoretical

Extracorporeal circulation

Blood activation during neonatal extracorporeal life support (Plötz et al). 1993;105:823-32

Extracorporeal membrane oxygenation

Plasma fentanyl levels in infants undergoing extracorporeal membrane oxygenation (Leuschen et al). 1993; 105:885-91

The reoxygenation phenomenon (Como and Samaja) (Letter); (Martin and Short) (Reply). 1993;105:373-4

F

Factor V

Immunization by bovine thrombin used with fibrin glue during cardiovascular operations: development of thrombin and factor V inhibitors (Bernuyer et al). 1993;105:892-7

Redo cardiac surgery: late bleeding complications from topical thrombin-induced factor $\mathrm{V}$ deficiency (Cmolik et al). 1993; $105: 222-8$ 
Factor IX deficiency; see Christmas disease

False-negative reactions

N2 lung cancer: outcome in patients with false-negative computed tomographic scans of the chest (Daly et al). 1993;105:904-11

\section{Femoral artery}

Relative risk of aortic and femoral insertion of intraaortic balloon pump after coronary artery bypass grafting procedures (Pinkard et al). 1993; 105:721-8

Fentanyl

Plasma fentanyl levels in infants undergoing extracorporeal mem-

Fetus brane oxygenation (Leuschen et al). 1993;105:885-91

Exclusion of the placenta during fetal cardiac bypass augments systemic flow and provides important information about the mechanism of placental injury (Fenton et al). 1993;-105:-502-12

Fibrillation, ventricular; see Ventricular fibrillation

Fibrin tissue adhesive

Immunization by bovine thrombin used with fibrin glue during cardiovascular operations: development of thrombin and factor V inhibitors (Bernuyer et al). 1993;105:892-7

Fibronectins

Changes in plasma fibronectin in children after elective repair of congenital heart defects (Hackbarth et al). 1993;105:31-6

Fibrous histiocytoma; see Histiocytoma

Filtration

Particle-induced coronary vasoconstriction: the need for in-line filtration of cardioplegic solutions (Palanzo and O'Neill) (Letter); (Rosenfeldt and Munsch) (Reply). 1993;105:1118-9

Fistula, coronary artery-pulmonary artery; see Arterio-arterial fistula

FK-506

A decade (1982 to 1992) of pediatric cardiac transplantation and the impact of FK 506 immunosuppression (Armitage et al). 1993; 105:464-73

Pediatric lung transplantation: the years 1985 to 1992 and the clinical trial of FK 506 (Armitage et al). 1993;105:337-46

Prolonged lung allograft survival with a short course of FK 506 (Hirai et al). 1993;105:1-8

Flavones

Flavone improves functional recovery after ischemia in isolated reperfused rabbit hearts (Ning et al). 1993;105:541-9

Fluorescence endoscopy; see Endoscopy

Fontan operation

Invited letter concerning: the importance of pulsatile flow when systemic venous retum is connected directly to the pulmonary arteries (Jonas) (Letter); (Muster and Mavroudis) (Reply). 1993; 105:173-6

Staged Fontan operation for complex cardiac anomalies with subaortic obstruction (Di Donato et al). 1993;105:398-405

Successful thrombectomy for thrombosis of the right side of the heart after the Fontan operation: report of two cases and review of the literature (Hedrick et al). 1993; 105:297-301

Foregut abnormalities; see Digestive system abnormalities

Free radicals

Time-course of free radical activity during coronary artery operations with cardiopulmonary bypass (Davies et al). 1993;105: 979-87

\section{$\boldsymbol{G}$}

\section{Gastroepiploic artery; see Arteries}

Gastroesophageal reflux

Functional foregut abnormalities in Barrett's esophagus (Stein et al). 1993;105:107-11

Invited letter concerning: technique for prevention of gastroesophageal reflux after transthoracic Heller's operation (Ellis) (Letter); (Gatzinsky et al) (Reply). 1993; 105:555-7
Technique for prevention of gastroesophageal reflux after transthoracic Heller's operation (Gatzinsky et al). 1993;105:553-5 (Letter)

Global ischemia; see Ischemia

Glucose

The optimal glucose concentration for intermittent cardioplegia in isolated rat heart when added to St. Thomas' Hospital cardioplegic solution (Owen et al). 1993;105:995-1006

Graft rejection

Breaking of transplantation tolerance after reduction of immunosuppression (Wijngaard et al). 1993;105:183-4 (Letter)

Cytoimmunologic monitoring for rejection and infection after lung transplantation (Schuurman et al). 1993;105:178-80 (Letter)

Invited letter concerning: transplantation tolerance and transplantation (George) (Letter); (Wijngaard et al) (Reply). 1993;105: 184-5

The sensitivity of transbronchial biopsy for the diagnosis of acute lung rejection (Tazelaar et al). 1993;105:674-8

Graft survival

Degeneration of aortic valve allografts in young recipients (Clarke et al). 1993;105:934-42

Effect of aprotinin (Trasylol) on aorta-coronary bypass graft patency (Bidstrup et al). 1993;105:147-53

Mechanical durability of pulmonary allograft conduits at systemic pressure: angiographic and histologic study in lambs (Kadoba et al). 1993;105:132-41

Prolonged lung allograft survival with a short course of FK 506 (Hirai et al). 1993;105:1-8

Surgical repair of type A aortic dissection by the circulatory arrest-graft inclusion technique in sixty-six patients (Galloway et al). 1993;105:781-90

Graham Traveling Fellowship

Graham Taveling Fellowship. 1993;105:572-3, 768, 954, 1123

H

Heart arrest, induced

Effect of calcium and preischemic hypothermia on recovery of myocardial function after cardioplegic ischemia in neonatal lambs (Aoki et al). 1993;105:207-13

Impaired endothelium-dependent coronary microvascular relaxation after cold potassium cardioplegia and reperfusion (Sellke et al). 1993;105:52-8

The microvascular distribution of cardioplegic solution in the piglet heart: retrograde versus antegrade delivery (Gates et al). $1993 ; 105: 845-53$

Myocardial distribution of cardioplegic solution after retrograde delivery in patients undergoing cardiac surgical procedures (Aronson et al). 1993; 105:214-21

Normocalcemic blood or crystalloid cardioplegia provides better neonatal myocardial protection than does low-calcium cardioplegia (Pearl et al). 1993;105:201-6

The optimal glucose concentration for intermittent cardioplegia in isolated rat heart when added to St. Thomas' Hospital cardioplegic solution (Owen et al). 1993;105:995-1006

Proton gradient during cardiac arrest: oxygenation of St. Thomas' Hospital cardioplegic solution and carbon dioxide level (Ichikawa and Yamamoto) (Letter); (von Oppell) (Reply). 1993; 105:551-3

Recovery of postischemic contractile function is depressed by antegrade warm continuous blood cardioplegia (Misare et al). 1993; 105:37-44

Retrograde versus antegrade delivery of cardioplegic solution in myocardial revascularization: a clinical trial in patients with three-vessel coronary artery disease who underwent myocardial revascularization with extensive use of the intemal mammary artery (Noyez et al). 1993;105:854-63 
Heart arrest, induced-cont'd

Surgical repair of type A aortic dissection by the circulatory arrest-graft inclusion technique in sixty-six patients (Galloway et al). 1993; 105:781-90

Ventricular function after normothermic versus hypothermic cardioplegia (Yau et al). 1993;105:833-44

Warm versus cold blood cardioplegia-Is there a difference? (Matsuura et al). 1993; 105:45-51

\section{Heart-assist devices}

Experience with an implantable tiered therapy device incorporating antitachycardia pacing and cardioverter/defibrillator therapy (Mitchell et al). 1993;105:453-63

A long-term ventricular assist system (Pierce et al). 1993;105:520-4

Heart contractility; see Myocardial contraction

Heart defects, congenital

Anatomic correction of atrioventricular discordance (Yamagishi et al). 1993;105:1067-76

Anatomic repair of transposition of great arteries with ventricular septal defect and aortic arch obstruction: one-stage versus twostage procedure (Planché et al). 1993;105:925-33

Biventricular repair of hypoplastic right ventricle assisted by pulsatile bidirectional cavopulmonary anastomosis (Muster et al). 1993; 105:112-9

Bless the babies: one hundred fifteen late survivors of heart transplantation during the first year of life (Bailey et al). 1993;105: 805-15

Changes in plasma fibronectin in children after elective repair of congenital heart defects (Hackbarth et al). 1993;105:31-6

A computer simulation of the plasma leakage through a vascular prosthesis made of expanded polytetrafluoroethylene (Tabata et al). 1993;105:598-604

Exclusion of the placenta during fetal cardiac bypass augments systemic flow and provides important information about the mechanism of placental injury (Fenton et al). 1993;105:502-12

Interleukin-8 release and neutrophil degranulation after pediatric cardiopulmonary bypass (Finn et al). 1993; 105:234-41

Intraventricular repair for Taussig-Bing anomaly (Kawashima et al). 1993;105:591-7

Isolated unilateral pulmonary artery agenesis (Canver). 1993;105: 766-7 (Letter)

The management of severe subaortic stenosis, ventricular septal defect, and aortic arch obstruction in the neonate (Bove et al). 1993;105:289-96

A new video-assisted thoracoscopic surgical technique for interruption of patent ductus arteriosus in infants and children (Laborde et al). 1993; 105:278-80

Outcomes in neonatal pulmonary atresia with intact ventricular septum: a multiinstitutional study (Hanley et al). 1993;105: 406-27

Repair of truncus arteriosus in the neonate (Hanley et al). 1993; 105:1047-56

Results of a policy of primary repair of truncus arteriosus in the neonate (Bove et al). 1993;105:1057-66

Results of surgical repair of congenital supravalvular aortic stenosis (Myers et al). 1993;105:281-8

Staged Fontan operation for complex cardiac anomalies with subaortic obstruction (Di Donato et al). 1993;105:398-405

Successful thrombectomy for thrombosis of the right side of the heart after the Fontan operation: report of two cases and review of the literature (Hedrick et al). 1993; 105:297-301

Superior function of a bicuspid over a monocuspid patch for reconstruction of a hypoplastic pulmonary root in pigs (Sievers et al). 1993;105:580-90
Heart failure, congestive

Prostaglandin $E_{1}$ for patients who have both heart and lung failure after cardiotomy (Mayumi and Tokunaga). 1993;105:1120-1 (Letter)

Heart-lung transplantation

A clinical trial of University of Wisconsin solution for pulmonary preservation (Hardesty et al). 1993; 105:660-6

An economic analysis of heart-lung transplantation: costs, insurance coverage, and reimbursement (Evans et al). 1993;105: 972-8

Improved ultrastructural lung preservation with prostaglandin $E_{1}$ as donor pretreatment in a primate model of heart-lung transplantation (Higgins et al). 1993;105:965-71

Heart preservation; see Organ preservation

Heart septal defects, ventricular

Anatomic repair of transposition of great arteries with ventricular septal defect and aortic arch obstruction: one-stage versus twostage procedure (Planché et al). 1993;105:925-33

The management of severe subaortic stenosis, ventricular septal defect, and aortic arch obstruction in the neonate (Bove et al) 1993; 105:289-96

Heart septum

Aneurysm of the membranous ventricular septum in transposition of the great arteries (Como et al). 1993;105:369-71 (Letter)

Outcomes in neonatal pulmonary atresia with intact ventricular septum: a multiinstitutional study (Hanley et al). 1993;105: 406-27

Heart surgery

Prostaglandin $E_{1}$ for patients who have both heart and lung failure after cardiotomy (Mayumi and Tokunaga). 1993;105:1120-1 (Letter)

Heart transplantation

Bless the babies: one hundred fifteen late survivors of heart transplantation during the first year of life (Bailey et al). 1993;105: 805-15

Breaking of transplantation tolerance after reduction of immunosuppression (Wijngaard et al). 1993; 105:183-4 (Letter)

A comparison of intracellular solutions for donor heart preservation (Hendry et al). 1993; 105:667-73

A decade (1982 to 1992) of pediatric cardiac transplantation and the impact of FK 506 immunosuppression (Amitage et al). 1993; 105:464-73

Improved recovery of heart transplants with a specific kit of preservation solutions (Menasché et al). 1993;105:353-63

Invited letter conceming: transplantation tolerance and transplantation (George) (Letter); (Wijngaard et al) (Reply). 1993;105: 184-5

Heart valve, transplantation

Inhibition of adenosine deaminase and nucleoside transport: utility in a model of homograft cardiac valve preimplantation processing (Abd-Elfattah et al). 1993;105:1095-105

Heart valve prosthesis

Clinical experience with the Omnicarbon prosthetic heart valve (Misawa et al). 1993;105:168-72

Effects of fixation back pressure and antimineralization treatment on the morphology of porcine aortic bioprosthetic valves (Flomenbaum and Schoer). 1993; 105:154-64

Late results of valve replacement with the Björk-Shiley valve (1973 to 1982) (Orszulak et al). 1993;105:302-12

Prosthetic valve endocarditis: experience with porcine bioprostheses (Sett et al). 1993;105:428-34

Prosthetic valve endocarditis with ring abscesses: surgical management and long-term results (Jault et al). 1993;105:1106-13

Sound spectral analysis of prosthetic valvular clicks for diagnosis of thrombosed Björk-Shiley tilting standard disc valve prostheses (Sato et al). 1993; 105:313-20 


\section{Heart ventricle}

Extensive cryoablation of the left ventricular posterior papillary muscle and subjacent ventricular wall: impact on mitral valve function and hemodynamics (Bakker et al). 1993;105:327-36

Is there an anatomic basis for subvalvular right ventricular outflow tract obstruction after an arterial switch repair for complete transposition? A morphometric study and review (Akiba et al). $1993 ; 105: 142-6$

Staged Fontan operation for complex cardiac anomalies with subaortic obstruction (Di Donato et al). 1993;105:398-405

Heart ventricle, right

Intraventricular repair for Taussig-Bing anomaly (Kawashima et al). 1993;105:591-7

Heller's operation; see Esophageal achalasia, surgery

Hemodilution

Acute isovolemic hemodilution and blood transfusion: effects on regional function and metabolism in myocardium with compromised coronary blood flow (Spahn et al). 1993; 105:694-704

Hemodynamics

Cardiac-derived thromboxane $A_{2}$ : An initiating mediator of reperfusion injury? (Byme et al). 1993;105:689-93

Extensive cryoablation of the left ventricular posterior papillary muscle and subjacent ventricular wall: impact on mitral valve function and hemodynamics (Bakker et al). 1993;105:327-36

Hemophilia B; see Christmas disease

Hemoptysis

Management and prognosis of massive hemoptysis: recent experience with 120 patients (Knott-Craig et al). 1993; 105:394-7

\section{Hemorrhage}

Comparison of two aprotinin dosage regimens in pediatric patients having cardiac operations: influence on platelet function and blood loss (Boldt et al). 1993;105:705-11

Effect of aprotinin (Trasylol) on aorta-coronary bypass graft patency (Bidstrup et al). 1993;105:147-53

Infusion of autologous platelet rich plasma does not reduce blood loss and product use after coronary artery bypass: a prospective, randomized, blinded study (Tobe et al). 1993;105:1007-14

Inhibition of platelet function by heparin: an etiologic factor in postbypass hemornhage (John et al). 1993;105:816-22

Redo cardiac surgery: late bleeding complications from topical thrombin-induced factor V deficiency (Cmolik et al). 1993; 105:222-8

Tranexamic acid (Cyklokapron) is not necessary to reduce blood loss after coronary artery bypass operations (Øvrum et al). $1993 ; 105: 78-83$

\section{Hemostatics}

Hemostatic activation during cardiopulmonary bypass with different aprotinin dosages in pediatric patients having cardiac operations (Dietrich et al). 1993; 105:712-20

The hemostatic effect of autologous platelet-rich plasma versus autologous whole blood after cardiac operations: Is platelet separation really necessary? (Mohr et al). 1993;105:371-3 (Letter)

Heparin

Inhibition of platelet function by heparin: an etiologic factor in postbypass hemorthage (John et al). 1993; 105:816-22

Hernia

Intrathoracic stomach: presentation and results of operation (Allen et al). 1993;105:253-9

\section{Hernia, hiatal}

Transhiatal esophagectomy for benign and malignant disease (Orringer et al). 1993; 105:265-77

\section{Histiocytoma}

Pleural effusion as the first manifestation of a malignant fibrous histiocytoma (García-Talavera et al). 1993;105:767 (Letter)

\section{Histology}

Mechanical durability of pulmonary allograft conduits at systemic pressure: angiographic and histologic study in lambs (Kadoba et al). 1993;105:132-41

Homograft cardiac valve; see Heart valve, transplantation

Hypertension, pulmonary

Selective pulmonary vasodilatation with inhaled nitric oxide (Tönz et al). 1993;105:760-2 (Letter)

\section{Hypertrophy}

Staged Fontan operation for complex cardiac anomalies with subaortic obstruction (Di Donato et al). 1993;105:398-405

Hypoplastic pulmonary root; see Pulmonary artery

Hypoplastic right ventricle; see Heart defects, congenital

Hypothermia, induced

Cold agglutinins and warm heart surgery (Gokhale et al). 1993; 105:557 (Letter)

Determinants of myocardial oxygen consumption in fibrillating dog hearts: comparison between normothermia and hypothermia (Yaku et al). 1993;105:679-88

Effect of calcium and preischemic hypothermia on recovery of myocardial function after cardioplegic ischemia in neonatal lambs (Aoki et al). 1993;105:207-13

Left ventricular pressure overload during postnatal development: effects on coronary vasodilator reserve and tolerance to hypothermic global ischemia (Yamamoto and Avkiran). 1993;105: 120-31

Hypothermic cardioplegia; see Heart arrest, induced

Hypothermic global ischemia; see Ischemia

Hypotonic solutions

Hypotonic cisplatin treatment for carcinomatous pleuritis found at thoracotomy in patients with lung cancer: in vitro experiments and preliminary clinical results (Ichinose et al). 1993;105: $1041-6$

Hypoxemia; see Anoxemia

Hypoxia; see Anoxia

Imaging fluorescence endoscopy; see Endoscopy

Immunity, cellular

Successful restoration of cell-mediated immune response after cardiopulmonary bypass by immunomodulation (Markewitz et al). 1993;105:15-24

Immunization

Immunization by bovine thrombin used with fibrin glue during cardiovascular operations: development of thrombin and factor V inhibitors (Berruyer et al). 1993;105:892-7

Immunomodulation; see Adjuvants, immunologic

Immunosuppression

Breaking of transplantation tolerance after reduction of immunosuppression (Wijngaard et al). 1993;105:183-4 (Letter)

A decade (1982 to 1992) of pediatric cardiac transplantation and the impact of FK 506 immunosuppression (Armitage et al). 1993; 105:464-73

Invited letter conceming: transplantation tolerance and transplantation (George) (Letter); (Wijngaard et al) (Reply). 1993;105: 184-5

Successful restoration of cell-mediated immune response after cardiopulmonary bypass by immunomodulation (Markewitz et al). 1993;105:15-24

Implantable tiered therapy device; see Heart assist devices

In memoriam

E. Stanley Crawford (1922-1992). 1993;105:579

Nina S. Braunwald (1928-1992) (Waldhausen). 1993;105:773-4 (Obit.) 


\section{Infant}

Bless the babies: one hundred fifteen late survivors of heart transplantation during the first year of life (Bailey et al). 1993;105: 805-15

A decade (1982 to 1992) of pediatric cardiac transplantation and the impact of FK 506 immunosuppression (Armitage et al). 1993; 105:464-73

Intraventricular repair for Taussig-Bing anomaly (Kawashima et al). 1993;105:591-7

Invited letter conceming: aprotinin use in pediatric cardiac operations (Edmunds) (Letter); (Dietrich and Mössinger) (Reply); (Boldt) (Reply). 1993; 105:757-60

Invited letter concerning: cardiac stun syndrome (Silverman). 1993; 105:374-5 (Letter)

Lung volumes, mechanics, and perfusion after pulmonary resection in infancy (Wemer et al). 1993;105:737-42

A new video-assisted thoracoscopic surgical technique for interruption of patent ductus arteriosus in infants and children (Laborde et al). 1993; 105:278-80

Pattems of ductal tissue in coarctation of the aorta in early infancy (van Son et al). 1993;105:368-9 (Letter)

Plasma fentanyl levels in infants undergoing extracorporeal membrane oxygenation (Leuschen et al). 1993; 105:885-91

The reoxygenation phenomenon (Corno and Samaja) (Letter); (Martin and Short) (Reply). 1993;105:373-4

Total anomalous pulmonary venous drainage by double connection corrected by ascending vein and coronary sinus repair (van de Wal). 1993;105:367-8 (Letter)

The use of "nonanatomic" pulmonary resection in infants with extensive congenital adenomatoid malformation of the lung (Browdie et al). 1993;105:732-6

\section{Infant, newborn}

Blood activation during neonatal extracorporeal life support (Plötz et al). 1993;105:823-32

Effect of calcium and preischemic hypothermia on recovery of myocardial function after cardioplegic ischemia in neonatal lambs (Aoki et al). 1993;105:207-13

Effects of high plasma epinephrine and $\mathrm{Ca}^{2+}$ concentrations on neonatal myocardial function after ischemia (Caspi et al). 1993;105:59-67

Invited letter conceming: critical aortic stenosis (McKay) (Letter); (Freedom) (Reply). 1993;105:365-7

Left ventricular pressure overload during postnatal development: effects on coronary vasodilator reserve and tolerance to hypothermic global ischemia (Yamamoto and Avkiran). 1993;105: 120-31

The management of severe subaortic stenosis, ventricular septal defect, and aortic arch obstruction in the neonate (Bove et al). 1993; 105:289-96

Normocalcemic blood or crystalloid cardioplegia provides better neonatal myocardial protection than does low-calcium cardioplegia (Pearl et al). 1993;105:201-6

Outcomes in neonatal pulmonary atresia with intact ventricular septum: a multiinstitutional study (Hanley et al). 1993;105: 406-27

The protective effect of magnesium on acute catecholamine cardiotoxicity in the neonate (Caspi et al). 1993;105:525-31

Repair of truncus arteriosus in the neonate (Hanley et al). 1993; 105:1047-56

Results of a policy of primary repair of truncus arteriosus in the neonate (Bove et al). 1993;105:1057-66

\section{Infection}

Cytoimmunologic monitoring for rejection and infection after lung transplantation (Schuurman et al). 1993;105:178-80 (Letter)

In situ repair of mycotic aneurysm of the ascending aorta (Pasic et al). 1993;105:321-6
Infusion of autologous platelets; see Blood component transfusion Inhalation administration; see Administration, inhalation

Innominate artery; see Brachiocephalic trunk

Insufflated talc; see Talc

Insurance, health, reimbursement

An economic analysis of heart-lung transplantation: costs, insurance coverage, and reimbursement (Evans et al). 1993;105: 972-8

Interleukin-8

Interleukin-8 release and neutrophil degranulation after pediatric cardiopulmonary bypass (Finn et al). 1993; 105:234-41

Intrazortic balloon pumping

Relative risk of aortic and femoral insertion of intraaortic balloon pump after coronary artery bypass grafting procedures (Pinkard et al). 1993; 105:721-8

Intracellul:sr solutions; see Cardioplegic solutions

Intraoperative care

An evaluation of the role of omentopexy and of early perioperative corticosteroid administration in clinical lung transplantation (Miller et al). 1993;105:247-52

Intraoperative use of inhaled low-dose nitric oxide (Miller et al). 1993; 105:550-1 (Letter)

Intraoperative complications

Cardiopulmonary dysfunction produced by reoxygenation of immature hypoxemic animals supported by cardiopulmonary bypass: prevention by intravenous metabolic pretreatment (Matheis et al). 1993:105:513-9

Intrathoracic meningocele; see Meningocele

Intrathoracic stomach; see Stomach diseases

Ischemia

Effect of calcium and preischemic hypothermia on recovery of myocardial function after cardioplegic ischemia in neonatal lambs (Aoki et al). 1993;105:207-13

Effects of 2,3-butanedione monoxime in isolated hearts: protection during reperfusion after global ischemia (Boban et al). 1993; 105:532-40

The effects of amrinone versus dobutamine on myocardial mechanics and energetics after hypothermic global ischemia (Ko et al). 1993;105:1015-24

Effects of high plasma epinephrine and $\mathrm{Ca}^{2+}$ concentrations on neonatal myocardial function after ischemia (Caspi et al). 1993;105:59-67

Flavone improves functional recovery after ischemia in isolated reperfused rabbit hearts (Ning et al). 1993;105:541-9

Left ventricular pressure overload during postnatal development: effects on coronary vasodilator reserve and tolerance to hypothermic global ischemia (Yamamoto and Avkiran). 1993;105: 120-31

Pathogenesis of ischemic mitral insufficiency (Llaneras et al). 1993;105:439-43

Recovery of postischemic contractile function is depressed by antegrade warm continuous blood cardioplegia (Misare et al). $1993 ; 105: 37-44$

Warm versus cold blood cardioplegia-Is there a difference? (Matsuura et al). 1993;105:45-51

Isosorbide dinitrate

Dilating effects of isosorbide dinitrate on diameter of intemal thoracic artery graft (Koike and Kimura). 1993;105:1121-2 (Letter)

Isovolemic hemodilution; see Hemodilution

L

Left ventricle; see Heart ventricle

Left ventricular function; see Ventricular function, left

Lelomyosarcoma

Leiomyosarcoma of the lung (Conte and Leitner). 1993;105:111920 (Letter) 


\section{Letters}

Letters. 1993;105:173-88, 364-78, 550-62, 757-67, 943-53, 1114-22

Lobectomy of lung; see Lung, surgery

Low-calcium cardioplegia; see Heart arrest, induced

Lung, metabolism

Changes in alveolar oxygen and carbon dioxide concentration and oxygen consumption during lung preservation: the maintenance of aerobic metabolism during lung preservation (Date et al). 1993; 105:492-501

Evaluation of lung metabolism during successful twenty-fourhour canine lung preservation (Date et al). 1993; 105:480-91

Lung, physiology

Physiologic evaluation of pulmonary function in the candidate for lung resection (Miller). 1993;105:347-52

Lung, surgery

Anatomic lobectomy of the lung by means of thoracoscopy: an experimental study (Kohno et al). 1993;105:729-31

Cardiac operation with associated pulmonary resection (Yokoyama et al). 1993; 105:912-7

Lung volumes, mechanics, and perfusion after pulmonary resection in infancy (Wemer et al). 1993;105:737-42

The use of "nonanatomic" pulmonary resection in infants with extensive congenital adenomatoid malformation of the lung (Browdie et al). 1993;105:732-6

Lung diseases

Differential lung ventilation: applications beyond the operating room (Adoumie et al). 1993; 105:229-33

Indications, risks, and results of completion pneumonectomy (Grégoire et al). 1993;105:918-24

Management and prognosis of massive hemoptysis: recent experience with 120 patients (Knott-Craig et al). 1993;105:394-7

Outcomes in neonatal pulmonary atresia with intact ventricular septum: a multiinstitutional study (Hanley et al). 1993;105: 406-27

Lung fallure; see Respiratory distress syndrome

Lung neoplasms

Anterior transcervical-thoracic approach for radical resection of lung tumors invading the thoracic inlet (Dartevelle et al). 1993;105:1025-34

Cardiac operation with associated pulmonary resection (Yokoyama et al). 1993; 105:912-7

Detection of dysplasia and carcinoma in situ with a lung imaging fluorescence endoscope device (Lam et al). 1993;105:1035-40

Hypotonic cisplatin treatment for carcinomatous pleuritis found at thoracotomy in patients with lung cancer: in vitro experiments and preliminary clinical results (lchinose et al). 1993;105: $1041-6$

Leiomyosarcoma of the lung (Conte and Leitner). 1993;105:1119. 20 (Letter)

N2 lung cancer: outcome in patients with false-negative computed tomographic scans of the chest (Daly et al). 1993;105:904-11

Surgical resection of stage IIIA and stage IIIB non-small-cell lung cancer after concurrent induction chemoradiotherapy: a Southwest Oncology Group trial (Rusch et al). 1993;105:97-106

Lung preservation; see Organ preservation

Lung transplantation

Bronchial circulation after experimental lung transplantation: the effect of long-term administration of prednisolone (Inui et al). 1993; 105:474-9

Changes in alveolar oxygen and carbon dioxide concentration and oxygen consumption during lung preservation: the maintenance of aerobic metabolism during lung preservation (Date et al). 1993;105:492-501

A clinical trial of University of Wisconsin solution for pulmonary preservation (Hardesty et al). 1993; 105:660-6
Cytoimmunologic monitoring for rejection and infection after lung transplantation (Schuuman et al). 1993;105:178-80 (Letter)

Evaluation of lung metabolism during successful twenty-fourhour canine lung preservation (Date et al). 1993;105:480-91

An evaluation of the role of omentopexy and of early perioperative corticosteroid administration in clinical lung transplantation (Miller et al). 1993; 105:247-52

Lung size matching for double lung transplantation based on the submammary thoracic perimeter: accuracy and functional results (Massard et al). 1993;105:9-14

Pediatric lung transplantation: the years 1985 to 1992 and the clinical trial of FK 506 (Amitage et al). 1993;105:337-46

Prolonged lung allograft survival with a short course of FK 506 (Hirai et al). 1993;105:1-8

The sensitivity of transbronchial biopsy for the diagnosis of acute lung rejection (Tazelaar et al). 1993;105:674-8

Lung ventilation; see Respiration, artificial

Lung volume measurements

Lung volumes, mcchanics, and perfusion after pulmonary resection in infancy (Wemer et al). 1993;105:737-42

\section{$\mathbf{M}$}

\section{Malignant pleural efiusion; see Pleural efiusion}

Mammary arteries

Retrograde versus antegrade delivery of cardioplegic solution in myocardial revascularization: a clinical trial in patients with three-vessel coronary artery disease who underwent myocardial revascularization with extensive use of the intemal mammary artery (Noyez et al). 1993;105:854-63

A simple and versatile sling stemal retractor for internal mammary artery harvesting (de Andrade Vicente). 1993;105:560-1 (Letter)

Thoracic wall necrosis in a patient with intemal mammary-coronary bypass after prosthetic replacement of the thoracoabdominal aorta (Aebert et al). 1993;105:557-8 (Letter)

Massive hemoptysis; see Hemoptysis

Maze procedure; see Surgery, methods

Mechanical ventilation; see Respiration, artificial

Mediastinum

Aneurysm of saphenous vein graft used for aorta-coronary bypass, resembling an anterior mediastinal mass (Robicsek et al). 1993;105:949-51 (Letter)

Meningocele

Intrathoracic meningocele (Auge and Georg). 1993;105:561-2 (Letter)

Mesothelioma

Mesothelioma: an incurable, nonsurgically treatable disease (Lewis) (Letter); (Rusch) (Reply). 1993;105:943-4

Microvasculature, see Blood vessels

Mitral valve

Extensive cryoablation of the left ventricular posterior papillary muscle and subjacent ventricular wall: impact on mitral valve function and hemodynamics (Bakker et al). 1993;105:327-36

Mitral valve insufirciency

Annuloplasty with flexible or rigid ring does not alter left ventricular systolic performance, energetics, or ventricular-arterial coupling in conscious, closed-chest dogs (Castro et al). 1993; 105:643-59

Left ventricular function in experimental mitral regurgitation with intact chordae tendineae (Hennein et al). 1993;105:624-32

Pathogenesis of ischemic mitral insufficiency (Llaneras et al). $1993 ; 105: 439-43$

Mitral valve stenosis

Long-term results of mitral commissurotomy (Scalia et al). 1993; 105:633-42 


\section{Models, theoretical}

Anatomic lobectomy of the lung by means of thoracoscopy: an experimental study (Kohno et al). 1993;105:729-31

Left ventricular function in experimental mitral regurgitation with intact chordae tendineae (Hennein et al). 1993;105:624-32

Monocuspid patch; see Bioprosthesis

Mortality

Clinical experience with the Omnicarbon prosthetic heart valve (Misawa et al). 1993;105:168-72

Muscles

Skeletal muscle-powered ventricle: effects of size and configuration on ventricular function (Oda et al). 1993;105:68-77

Mycotic aneurysm; see Aneurysm, infected

Myocardial contraction

Effects of hypoxia on intracellular calcium and contractility (Urthaler et al). 1993;105:1114-6 (Letter)

Recovery of postischemic contractile function is depressed by antegrade warm continuous blood cardioplegia (Misare et al). 1993; 105:37-44

Redistribution of myocardial calcium during ischemia: relationship to onset of contracture (Jimenez et al). 1993;105:988-94

Myocardial infarction

Pathogenesis of ischemic mitral insufficiency (Llaneras et al). $1993 ; 105: 439-43$

Myocardial ischemia

Redistribution of myocardial calcium during ischemia: relationship to onset of contracture (Jimenez et al). 1993;105:988-94

Myocardial reperfusion

Effects of 2,3-butanedione monoxime in isolated hearts: protection during reperfusion after global ischemia (Boban et al). 1993; 105:532-40

Flavone improves functional recovery after ischemia in isolated reperfused rabbit hearts (Ning et al). 1993;105:541-9

Impaired endothelium-dependent coronary microvascular relaxation after cold potassium cardioplegia and reperfusion (Sellke et al). 1993;105:52-8

Superiority of controlled surgical reperfusion versus percutaneous transluminal coronary angioplasty in acute coronary occlusion (Allen et al). 1993;105:864-84

\section{Myocardial revascularization}

Retrograde versus antegrade delivery of cardioplegic solution in myocardial revascularization: a clinical trial in patients with three-vessel coronary artery disease who underwent myocardial revascularization with extensive use of the internal mammary artery (Noyez et al). 1993; 105:854-63

Myocardium

Myocardial distribution of cardioplegic solution after retrograde delivery in patients undergoing cardiac surgical procedures (Aronson et al). 1993;105:214-21

Normocalcemic blood or crystalloid cardioplegia provides better neonatal myocardial protection than does low-calcium cardioplegia (Pearl et al). 1993;105:201-6

Myocardium, drug effects

The effects of amrinone versus dobutamine on myocardial mechanics and energetics after hypothermic global ischemia (Ko et al). 1993;105:1015-24

Myocardium, injuries

Invited letter concerning: cardiac stun syndrome (Silverman). 1993; 105:374-5 (Letter)

The reoxygenation phenomenon (Como and Samaja) (Letter); (Martin and Short) (Reply). 1993;105:373-4

Myocardium, metabolism

Acute isovolemic hemodilution and blood transfusion: effects on regional function and metabolism in myocardium with compromised coronary blood flow (Spahn et al). 1993; 105:694-704
Determinants of myocardial oxygen consumption in fibrillating dog hearts: comparison between normothermia and hypothermia (Yaku et al). 1993;105:679-88

\section{Myocardium, physiology}

Effect of calcium and preischemic hypothermia on recovery of myocardial function after cardioplegic ischemia in neonatal lambs (Aoki et al). 1993;105:207-13

Effects of high plasma epinephrine and $\mathrm{Ca}^{2+}$ concentrations on neonatal myocardial function after ischemia (Caspi et al). 1993;105:59-67

Myocardium, ultrastructure

Reply to invited letter conceming: assessment of myocardial ultrastructure after retrograde infusion of cardioplegic solution ( $\mathrm{J}$ Thorac Cardiovasc Surg 1992;104:1745-6) (Fischer and Bamer). 1993;105:762-3 (Letter)

\section{$\mathbf{N}$}

Necrosis

Thoracic wall necrosis in a patient with intemal mammary-coronary bypass after prosthetic replacement of the thoracoabdominal aorta (Aebert et al). 1993;105:557-8 (Letter)

Neointima; see Blood vessels

Neonate; see Infant, newborn

Neoplasms

Medical tumors of the chest wall: solitary plasmacytoma and Ewing's sarcoma (Burt et al). 1993; 105:89-96

Solitary bone plasmacytoma of rib presenting as a superior sulcus tumor (Rocco et al). 1993;105:944-5 (Letter)

Neurofibromatosis I

Intrathoracic meningocele (Auge and Georg). 1993;105:561-2 (Letter)

Neutrophils

Effect of cardiopulmonary bypass on systemic release of neutrophil elastase and tumor necrosis factor (Butler et al). 1993; 105:25-30

Interleukin-8 release and neutrophil degranulation after pediatric cardiopulmonary bypass (Finn et al). 1993;105:234-41

Reply to: neutrophil activation during cardiopulmonary bypass (J ThORAC CaRdiovasC SuRg 1992;104:1746-8) (Dreyer et al). 1993;105:763 (Letter)

Newborn; see Infant, newborn

Nitric oxide

Intraoperative use of inhaled low-dose nitric oxide (Miller et al). 1993;105:550-1 (Letter)

Selective pulmonary vasodilatation with inhaled nitric oxide (Tönz et al). 1993; 105:760-2 (Letter)

Non-small cell lung carcinoma; see Carcinoma, non-small cell lung

Normocalcemic blood cardioplegia; see Heart arrest, induced

Normothermic cardioplegia; see Heart arrest, induced

Nucleosides

Inhibition of adenosine deaminase and nucleoside transport: utility in a model of homograft cardiac valve preimplantation processing (Abd-Elfattah et al). 1993;105:1095-105

\section{0}

\section{Obituaries}

E. Stanley Crawford (1922-1992). 1993;105:579

Nina S. Braunwald (1928-1992) (Waldhausen). 1993;105:773-4

\section{Omentum}

An evaluation of the role of omentopexy and of early perioperative corticosteroid administration in clinical lung transplantation (Miller and DeHoyos). 1993;105:247-52 


\section{Omnicarbon prosthetic valve; see Heart valve prosthesis \\ Organ preservation}

Changes in alveolar oxygen and carbon dioxide concentration and oxygen consumption during lung preservation: the maintenance of aerobic metabolism during lung preservation (Date et al). 1993;105:492-501

A clinical trial of University of Wisconsin solution for pulmonary preservation (Hardesty et al). 1993; 105:660-6

A comparison of intracellular solutions for donor heart preservation (Hendry et al). 1993; 105:667-73

Evaluation of lung metabolism during successful twenty-fourhour canine lung preservation (Date et al). 1993; 105:480-91

Improved recovery of heart transplants with a specific kit of preservation solutions (Menasché et al). 1993;105:353-63

Improved ultrastructural lung preservation with prostaglandin $E_{1}$ as donor pretreatment in a primate model of heart-lung transplantation (Higgins et al). 1993;105:965-71

Organ weight

Lung size matching for double lung transplantation based on the submammary thoracic perimeter: accuracy and functional results (Massard et al). 1993;105:9-14

Oxygen

Cardiopulmonary dysfunction produced by reoxygenation of immature hypoxemic animals supported by cardiopulmonary bypass: prevention by intravenous metabolic pretreatment (Matheis et al). 1993;105:513-9

Proton gradient during cardiac arrest: oxygenation of St. Thomas' Hospital cardioplegic solution and carbon dioxide level (Ichikawa and Yamamoto) (Letter); (von Oppell) (Reply). 1993; 105:551-3

Oxygen consumption

Changes in alveolar oxygen and carbon dioxide concentration and oxygen consumption during lung preservation: the maintenance of aerobic metabolism during lung preservation (Date et al). 1993;105:492-501

Determinants of myocardial oxygen consumption in fibrillating dog hearts: comparison between normothermia and hypothermia (Yaku et al). 1993;105:679-88

\section{$\mathbf{p}$}

Pacing wires; see Cardiac pacing, artificial

Pancreatopeptidase

Effect of cardiopulmonary bypass on systemic release of neutrophil elastase and tumor necrosis factor (Butler et al). 1993; 105:25-30

Papillary muscles

Extensive cryoablation of the left ventricular posterior papillary muscle and subjacent ventricular wall: impact on mitral valve function and hemodynamics (Bakker et al). 1993;105:327-36

Patent ductus arteriosus; see Ductus arteriosus, patent

Pediatrics; see Child; Infant

Percutaneous transluminal coronary angioplasty; see Angioplasty, transluminal, percutaneous coronary

Pericardial flap; see Surgical flaps

Pericardium

Preliminary experimental results of a new resorbable biomaterial as pericardial substitute (Fradin et al). 1993;105:364-5 (Letter)

Perimetry

Lung size matching for double lung transplantation based on the submammary thoracic perimeter: accuracy and functional results (Massard et al). 1993;105:9-14

Perioperative care; see Intraoperative care

Placenta

Exclusion of the placenta during fetal cardiac bypass augments systemic flow and provides important information about the mechanism of placental injury (Fenton et al). 1993;105:502-12
Plasma

Changes in plasma fibronectin in children after elective repair of congenital heart defects (Hackbarth et al). 1993;105:31-6

A computer simulation of the plasma leakage through a vascular prosthesis made of expanded polytetrafluoroethylene (Tabata et al). 1993;105:598-604

Effects of high plasma epinephrine and $\mathrm{Ca}^{2+}$ concentrations on neonatal myocardial function after ischemia (Caspi et al). 1993;105:59-67

The hemostatic effect of autologous platelet-rich plasma versus autologous whole blood after cardiac operations: Is platelet separation really necessary? (Mohr et al). 1993;105:371-3 (Letter)

Infusion of autologous platelet rich plasma does not reduce blood loss and product use after coronary artery bypass: a prospective, randomized, blinded study (Tobe et al). 1993;105:1007-14

Plasma fentanyl levels in infants undergoing extracorporeal membrane oxygenation (Leuschen et al). 1993;105:885-91

Plasma levels of endothelin-1 and thrombin-antithrombin III complex in patients undergoing open chest operations (Onizuka et al). 1993;105:559-60 (Letter)

Reply to invited letter conceming: changes in plasma-free thyroid hormones during cardiopulmonary bypass (J THORAC CARDIOVASC SURG 1992;104:526-7) (Gøtzsche and Weeke). 1993; 105:176-8 (Letter)

\section{Plasmacytoma}

Medical tumors of the chest wall: solitary plasmacytoma and Ewing's sarcoma (Burt et al). 1993;105:89-96

Solitary bone plasmacytoma of rib presenting as a superior sulcus tumor (Rocco et al). 1993;105:944-5 (Letter)

Platelet activation

Blood activation during neonatal extracorporeal life support (Plötz et al). 1993;105:823-32

Platelet function tests

Comparison of two aprotinin dosage regimens in pediatric patients having cardiac operations: influence on platelet function and blood loss (Boldt et al). 1993;105:705-11

Platelets; see Blood platelets

Pleura

Thoracoscopic pleurectomy for treatment of complicated spontaneous pneumothorax (Inderbitzi et al). 1993;105:84-8

Pleural effusion

Comparison of insufflated talc under thoracoscopic guidance with standard tetracycline and bleomycin pleurodesis for control of malignant pleural effusions (Hartman et al). 1993;105:743-8

Pleural effusion as the first manifestation of a malignant fibrous histiocytoma (García-Talavera et al). 1993;105:767 (Letter)

Pleurisy

Hypotonic cisplatin treatment for carcinomatous pleuritis found at thoracotomy in patients with lung cancer: in vitro experiments and preliminary clinical results (Ichinose et al). 1993;105: 1041-6

Pneumonectomy

Indications, risks, and results of completion pneumonectomy (Grégoire et al). 1993;105:918-24

Mesothelioma: an incurable, nonsurgically treatable disease (Lewis) (Letter); (Rusch) (Reply). 1993;105:943-4

Physiologic evaluation of pulmonary function in the candidate for lung resection (Miller). 1993;105:347-52

\section{Pneumothorax}

Thoracoscopic pleurectomy for treatment of complicated spontaneous pneumothorax (Inderbitzi et al). 1993; 105:84-8

Thoracoscopic stapled resection for spontaneous pneumothorax (Hazelrigg et al). 1993;105:389-93 
Polytetrafluoroethylene

A computer simulation of the plasma leakage through a vascular prosthesis made of expanded polytetrafluoroethylene (Tabata et al). 1993; 105:598-604

Polyurethanes

Very small-diameter polyurethane vascular prostheses with rapid endothelialization for coronary artery bypass grafting (Okoshi et al). 1993;105:791-5

Porcine bioprosthesis; see Bioprosthesis

Posterior aorta; see Aorta

Postnatal development; see Infant, newborn

Postoperative complications

Invited letter conceming: technique for prevention of gastroesophageal reflux after transthoracic Heller's operation (Ellis) (Letter); (Gatzinsky et al) (Reply). 1993; 105:555-7

Technique for prevention of gastroesophageal reflux after transthoracic Heller's operation (Gatzinsky et al). 1993;105:553-5 (Letter)

Postoperative period

Selection of patients for same-day coronary bypass operations (Anderson et al). 1993;105:444-52

Postoperative wound infection; see Surgical wound infection

Potassium

Impaired endothelium-dependent coronary microvascular relaxation after cold potassium cardioplegia and reperfusion (Sellke et al). 1993;105:52-8

Prednisolone

Bronchial circulation after experimental lung transplantation: the effect of long-term administration of prednisolone (Inui et al) $1993 ; 105: 474-9$

Preoperative care

Cardiopulmonary dysfunction produced by reoxygenation of immature hypoxemic animals supported by cardiopulmonary bypass: prevention by intravenous metabolic pretreatment (Matheis et al). 1993;105:513-9

Preservation solutions; see Organ preservation

Presidential address

Fulfilling expectations (Fosburg). 1993;105:194-200

Primate model; see Disease models, animal

Prostaglandin E1; see Alprostadil

Prosthesis failure

Effects of fixation back pressure and antimineralization treatment on the morphology of porcine aortic bioprosthetic valves (Flomenbaum and Schoen). 1993; 105:154-64

Late results of valve replacement with the Björk-Shiley valve (1973 to 1982) (Orszulak et al). 1993;105:302-12

Sound spectral analysis of prosthetic valvular clicks for diagnosis of thrombosed Björk-Shiley tilting standard disc valve prostheses (Sato et al). 1993;105:313-20

Prosthesis-related infections

Prosthetic valve endocarditis: experience with porcine bioprostheses (Sett et al). 1993;105:428-34

Prosthetic valve endocarditis with ring abscesses: surgical management and long-term results (Jault et al). 1993;105:1106-13

Protons

Proton gradient during cardiac arrest: oxygenation of St. Thomas' Hospital cardioplegic solution and carbon dioxide level (Ichikawa and Yamamoto) (Letter); (von Oppell) (Reply) 1993; 105:551-3

Pulmonary artery

Invited letter conceming: the importance of pulsatile flow when systemic venous retum is connected directly to the pulmonary arteries (Jonas) (Letter); (Muster and Mavroudis) (Reply). 1993;105:173-6

Isolated unilateral pulmonary artery agenesis (Canver). 1993;105: 766-7 (Letter)
Mechanical durability of pulmonary allograft conduits at systemic pressure: angiographic and histologic study in lambs (Kadoba et al). 1993; 105:132-41

Superior function of a bicuspid over a monocuspid patch for reconstruction of a hypoplastic pulmonary root in pigs (Sievers et al). 1993;105:580-90

Tetralogy of Fallot with pulmonary atresia, coronary arterypulmonary artery fistula, and origin of left pulmonary artery from descending aorta: total correction in infancy (Metras et al). 1993; 105:186-8 (Letter)

Pulmonary atresia; see Lung diseases

Pulmonary function tests; see Respiratory function tests

Pulmonary hypertension; see Hypertension, pulmonary

Pulmonary preservation; see Organ preservation

Pulmonary resection; see Lung, surgery

Pulmonary root

Time course of dimension and function of the autologous pulmonary root in the aortic position (Sievers et al). 1993;105:775-80

Pulmonary valve stenosis

Surgical correction of a recurrent aneurysm of the ascending aorta simulating pulmonary stenosis (Rabajoli et al). 1993;105:9489 (Letter)

Pulmonary veins

Total anomalous pulmonary venous drainage by double connection corrected by ascending vein and coronary sinus repair (van de Wal). 1993;105:367-8 (Letter)

Pulsatile flow

Invited letter conceming: the importance of pulsatile flow when systemic venous retum is connected directly to the pulmonary arteries (Jonas) (Letter); (Muster and Mavroudis) (Reply). 1993; 105:173-6

Quality improvement

Fulfilling expectations (Fosburg). 1993;105:194-200 (Pres. address)

Radiotherapy

Surgical resection of stage IIIA and stage IIIB non-small-cell lung cancer after concurrent induction chemoradiotherapy: a Southwest Oncology Group trial (Rusch et al). 1993;105:97-106

Regional blood flow

Exclusion of the placenta during fetal cardiac bypass augments systemic flow and provides important information about the mechanism of placental injury (Fenton et al). 1993;105:502-12

Reimbursement; see Insurance, health, reimbursement

Relative risk; see Risk factors

Reoperation

The effect of coronary reoperation on the survival of patients with stenoses in saphenous vein bypass grafts to coronary arteries (Lytle et al). 1993; 105:605-14

Indications, risks, and results of completion pneumonectomy (Grégoire et al). 1993;105:918-24

Redo cardiac surgery: late bleeding complications from topical thrombin-induced factor $\mathrm{V}$ deficiency (Cmolik et al). 1993; 105:222-8

Reoxygenation; see Extracorporeal membrane oxygenation; Oxygen

Reperfused hearts; see Myocardial reperfusion

Reperfusion injury

Cardiac-derived thromboxane $\mathrm{A}_{2}$ : An initiating mediator of reperfusion injury? (Byme et al). 1993;105:689-93

Respiration, artificial

Differential lung ventilation: applications beyond the operating room (Adoumie et al). 1993; 105:229-33 


\section{Respiratory distress syndrome}

Prostaglandin $E_{1}$ for patients who have both heart and lung failure after cardiotomy (Mayumi and Tokunaga). 1993;105:1120-1 (Letter)

\section{Respiratory function tests}

Physiologic evaluation of pulmonary function in the candidate for lung resection (Miller). 1993;105:347-52

Retrograde cardioplegia; see Heart arrest, induced

Revascularization; see Blood vessels

Ribs

Solitary bone plasmacytoma of rib presenting as a superior sulcus tumor (Rocco et al). 1993;105:944-5 (Letter)

Right ventricular function; see Ventricular function, right

Ring abscess; see Abscess

Risk factors

Indications, risks, and results of completion pneumonectomy (Grégoire et al). 1993;105:918-24

Relative risk of aortic and femoral insertion of intraaortic balloon pump after coronary artery bypass grafting procedures (Pinkard et al). 1993; 105:721-8

\section{s}

St. Thomas Hospital cardioplegic solution; see Cardioplegic solutions

Same-day surgery

Selection of patients for same-day coronary bypass operations (Anderson et al). 1993;105:444-52

Saphenous vein

Aneurysm of saphenous vein graft used for aorta-coronary bypass, resembling an anterior mediastinal mass (Robicsek et al). 1993;105:949-51 (Letter)

The effect of coronary reoperation on the survival of patients with stenoses in saphenous vein bypass grafts to coronary arteries (Lytle et al). 1993; 105:605-14

Sarcoma, Ewing's

Medical tumors of the chest wall: solitary plasmacytoma and Ewing's sarcoma (Burt et al). 1993; 105:89-96

Size matching; see Organ weight

Skeletal muscles; see Muscles

Sling sternal retractor; see Surgical equipment

Sound spectral analysis; see Spectrum analysis

Southwest Oncology Group

Surgical resection of stage IIIA and stage IIIB non-small-cell lung cancer after concurrent induction chemoradiotherapy: a Southwest Oncology Group trial (Rusch et al). 1993;105:97-106

Spectrum analysis

Sound spectral analysis of prosthetic valvular clicks for diagnosis of thrombosed Björk-Shiley tilting standard disc valve prostheses (Sato et al). 1993; 105:313-20

Staplers; see Surgical staplers

Statement of appreciation

Statement of appreciation. 1993; 105:193

Stomach diseases

Intrathoracic stomach: presentation and results of operation (Allen et al). 1993; 105:253-9

Stroke volume

Skeletal muscle-powered ventricle: effects of size and configuration on ventricular function (Oda et al). 1993;105:68-77

Subaortic stenosis; see Aortic valve stenosis

Subclavian artery

Aortic aneurysm after subclavian arterial flap angioplasty for coarctation of the aorta (Berri et al). 1993; 105:951 (Letter)

Dysphagia lusoria (I̊ğci et al). 1993;105:1116-8 (Letter)
Submammary thoracic perimeter; see Perimetry

Subvalvular right ventricular outflow tract obstruction; see Ventricular outflow tract obstruction

Sulcus tumors; see Neoplasms

Superior vena cava; see Vena cava, superior

Supravelvular aortic stenosis; see Aortic valve stenosis

Surgery, methods

Initial experience with the maze procedure for atrial fibrillation (McCarthy et al). 1993; 105:1077-87

Surgical equipment

Anatomic lobectomy of the lung by means of thoracoscopy: an experimental study (Kohno et al). 1993;105:729-31

A simple and versatile sling stemal retractor for intemal mammary artery harvesting (Villela de Andrade Vicente). 1993;105:5601 (Letter)

Surgical flaps

Aortic aneurysm after subclavian arterial flap angioplasty for coarctation of the aorta (Berri et al). 1993; 105:951 (Letter)

Blunt injury of the innominate artery: use of a vascular pericardial flap (Asimacopoulos et al). 1993;105:764-5 (Letter)

Surgical staplers

Thoracoscopic stapled resection for spontaneous pneumothorax (Hazelrigg et al). 1993;105:389-93

Surgical wound infection

Clinical significance of epicardial pacing wire cultures (Hastings and Robicsek). 1993;105:165-7

Survival

Actuarial five-year survival estimates (Mengoli) (Letter); (Dartevelle and Macchiarini) (Reply). 1993;105:375-7

The effect of coronary reoperation on the survival of patients with stenoses in saphenous vein bypass grafts to coronary arteries (Lytle et al). 1993;105:605-14

Endoscopic surveillance of Barrett's esophagus: Does it help? (Streitz et al). 1993;105:383-8

Late results of valve replacement with the Björk-Shiley valve (1973 to 1982) (Orszulak et al). 1993;105:302-12

Repair of truncus arteriosus in the neonate (Hanley et al). 1993; 105:1047-56

Results of a policy of primary repair of truncus arteriosus in the neonate (Bove et al). 1993;105:1057-66

Survival of patients with carcinoma of the esophagus treated with combined-modality therapy (Wolfe et al). 1993;105:749-56

Survival rate

Bless the babies: one hundred fifteen late survivors of heart transplantation during the first year of life (Bailey et al). 1993; 105: 805-15

Clinical experience with the Omnicarbon prosthetic heart valve (Misawa et al). 1993;105:168-72

Systemic flow; see Regional blood flow

Systole

Annuloplasty with flexible or rigid ring does not alter left ventricular systolic performance, energetics, or ventricular-arterial coupling in conscious, closed-chest dogs (Castro et al). 1993; 105:643-59

T

\section{Tachycardia}

Experience with an implantable tiered therapy device incorporating antitachycardia pacing and cardioverter/defibrillator therapy (Mitchell et al). 1993;105:453-63

Extensive cryoablation of the left ventricular posterior papillary muscle and subjacent ventricular wall: impact on mitral valve function and hemodynamics (Bakker et al). 1993;105:327-36 
Talc

Comparison of insufflated talc under thoracoscopic guidance with standard tetracycline and bleomycin pleurodesis for control of malignant pleural effusions (Hartman et al). 1993;105:743-8

Taussig-Bing anomaly

Intraventricular repair for Taussig-Bing anomaly (Kawashima et al). 1993;105:591-7

Tetracycline

Comparison of insufflated talc under thoracoscopic guidance with standard tetracycline and bleomycin pleurodesis for control of malignant pleural effusions (Hartman et al). 1993; 105:743-8

Tetralogy of Fallot

Tetralogy of Fallot with pulmonary atresia, coronary arterypulmonary artery fistula, and origin of left pulmonary artery from descending aorta: total correction in infancy (Metras et al). 1993;105:186-8 (Letter)

\section{Thoracic arteries}

Clinical evaluation with exercise performance in twenty patients who underwent coronary artery bypass grafting with both the gastroepiploic and intemal thoracic arteries (Isomura et al). 1993;105:1088-94

Dilating effects of isosorbide dinitrate on diameter of intemal thoracic artery graft (Koike and Kimura). 1993;105:1121-2 (Letter)

\section{Thoracic diseases}

Transhiatal esophagectomy for benign and malignant disease (Orringer et al). 1993; 105:265-77

Thoracic esophageal diverticulum; see Esophageal diverticulum

Thoracic neoplasms

Medical tumors of the chest wall: solitary plasmacytoma and Ewing's sarcoma (Burt et al). 1993;105:89-96

Thoracic perimeter, submammary; see Perimetry

Thoracic wall necrosis; see Necrosis

Thoracoabdominal aorta; see Aorta, abdominal; Aorta, thoracic

Thoracoscopy

Anatomic lobectomy of the lung by means of thoracoscopy: an experimental study (Kohno et al). 1993;105:729-31

Comparison of insufflated talc under thoracoscopic guidance with standard tetracycline and bleomycin pleurodesis for control of malignant pleural effusions (Hartman et al). 1993; 105:743-8

A new video-assisted thoracoscopic surgical technique for interruption of patent ductus arteriosus in infants and children (Laborde et al). 1993; 105:278-80

Thoracoscopic pleurectomy for treatment of complicated spontaneous pneumothorax (Inderbitzi et al). 1993; 105:84-8

Thoracoscopic stapled resection for spontaneous pneumothorax (Hazelrigg et al). 1993;105:389-93

\section{Thoracotomy}

Esophagectomy with or without thoracotomy: Is there any difference? (Tilanus et al). 1993;105:898-903

Hypotonic cisplatin treatment for carcinomatous pleuritis found at thoracotomy in patients with lung cancer: in vitro experiments and preliminary clinical results (Ichinose et al). 1993;105: $1041-6$

Thoracoscopic stapled resection for spontaneous pneumothorax (Hazelrigg et al). 1993;105:389-93

Thorax

Anterior transcervical-thoracic approach for radical resection of lung tumors invading the thoracic inlet (Dartevelle et al). 1993; 105: 1025-34

N2 lung cancer: outcome in patients with false-negative computed tomographic scans of the chest (Daly et al). 1993;105:904-11
Thrombectomy; see Thrombosis

Thrombin

Immunization by bovine thrombin used with fibrin glue during cardiovascular operations: development of thrombin and factor $\mathrm{V}$ inhibitors (Berruyer et al). 1993;105:892-7

Plasma levels of endothelin-1 and thrombin-antithrombin III complex in patients undergoing open chest operations (Onizuka et al). 1993;105:559-60 (Letter)

Redo cardiac surgery: late bleeding complications from topical thrombin-induced factor V deficiency (Cmolik et al). 1993; 105:222-8

Thrombosis

Sound spectral analysis of prosthetic valvular clicks for diagnosis of thrombosed Björk-Shiley tilting standard disc valve prostheses (Sato et al). 1993;105:313-20

Successful thrombectomy for thrombosis of the right side of the heart after the Fontan operation: report of two cases and review of the literature (Hedrick et al). 1993;105:297-301

Thromboxane A2

Cardiac-derived thromboxane $A_{2}$ : An initiating mediator of reperfusion injury? (Byme et al). 1993;105:689-93

Thyroid gland

Allograft replacement of the trachea: experimental synchronous revascularization of composite thyrotracheal transplant (Khalil-Marzouk). 1993;105:242-6

\section{Thyrold hormones}

Reply to invited letter conceming: changes in plasma-free thyroid hormones during cardiopulmonary bypass (J THORAC CARDIOVASC SURG 1992;104:526-7) (Gøtzsche and Weeke). 1993; 105:176-8 (Letter)

Thyrotrachael transplant; see Trachea, transplantation

Tissue donors

Improved ultrastructural lung preservation with prostaglandin $\mathrm{E}_{1}$ as donor pretreatment in a primate model of heart-lung transplantation (Higgins et al). 1993;105:965-71

Lung size matching for double lung transplantation based on the submammary thoracic perimeter: accuracy and functional results (Massard et al). 1993;105:9-14

Tissue transplantation

Acceleration of neointima formation in vascular prostheses by transplantation of autologous venous tissue fragments: application to small-diameter grafts (Noishiki et al). 1993;105: 796-804

Tomography, x-ray computed

N2 lung cancer: outcome in patients with false-negative computed tomographic scans of the chest (Daly et al). 1993;105:904-11

Trachea, transplantation

Allograft replacement of the trachea: experimental synchronous revascularization of composite thyrotracheal transplant (Khalil-Marzouk). 1993;105:242-6

Tranexamic acid

Tranexamic acid (Cyklokapron) is not necessary to reduce blood loss after coronary artery bypass operations (Øvrum et al). 1993;105:78-83

Transbronchial blopsy; see Biopsy

Transcervical approach; see Thorax

Transesophageal echocardlography; see Echocardiography

Transhiatal esophagectomy; see Esophagectomy

Transplantation, autologous

Acceleration of neointima formation in vascular prostheses by transplantation of autologous venous tissue fragments: application to small-diameter grafts (Noishiki et al). 1993;105: 796-804

Time course of dimension and function of the autologous pulmonary root in the aortic position (Sievers et al). 1993;105:775-80 
Transplantation, homologous

Allograft replacement of the trachea: experimental synchronous revascularization of composite thyrotracheal transplant (Khalil-Marzouk). 1993;105:242-6

Degeneration of aortic valve allografts in young recipients (Clarke et al). 1993;105:934-42

Mechanical durability of pulmonary allograft conduits at systemic pressure: angiographic and histologic study in lambs (Kadoba et al). 1993;105:132-41

Prolonged lung allograft survival with a short course of FK 506 (Hirai et al). 1993; 105:1-8

Transplantation immunology

Breaking of transplantation tolerance after reduction of immunosuppression (Wijngaard et al). 1993; 105:183-4 (Letter)

Invited letter concerning: transplantation tolerance and transplantation (George) (Letter); (Wijngaard et al) (Reply). 1993;105: 184-5

Transport, blological; see Blological transport

Transposition of great vessels

Anatomic repair of transposition of great arteries with ventricular septal defect and aortic arch obstruction: one-stage versus twostage procedure (Planché et al). 1993;105:925-33

Aneurysm of the membranous ventricular septum in transposition of the great arteries (Como et al). 1993;105:369-71 (Letter)

Is there an anatomic basis for subvalvular right ventricular outflow tract obstruction after an arterial switch repair for complete transposition? A morphometric study and review (Akiba et al). 1993;105:142-6

Transposition of the great arteries with posterior aorta (Antunes). 1993;105:369 (Letter)

Transthoracic Heller's operation; see Esophageal achalasia, surgery

Trasylol; see Aprotinin

Truncus arteriosus

Repair of truncus arteriosus in the neonate (Hanley et al). 1993; 105:1047-56

Results of a policy of primary repair of truncus arteriosus in the neonate (Bove et al). 1993;105:1057-66

Tuberculosis, pulmonary

Management and prognosis of massive hemoptysis: recent experience with 120 patients (Knott-Craig et al). 1993; 105:394-7

Tumor necrosis factor

Effect of cardiopulmonary bypass on systemic release of neutrophil elastase and tumor necrosis factor (Butler et al). 1993; 105:25-30

Tumors; see Neoplasms

Type A aortic dissection; see Aneurysm, dissecting

u

University of Wisconsin solution; see Cardioplegic solutions

Vascular pericardial flap; see Surgical flaps

Vascular prosthesis; see Blood vessel prosthesis

Vasoconstriction

Particle-induced coronary vasoconstriction: the need for in-line filtration of cardioplegic solutions (Palanzo and O'Neill) (Letter); (Rosenfeldt and Munsch) (Reply). 1993;105:1118-9

\section{Vasodilation}

Left ventricular pressure overload during postnatal development: effects on coronary vasodilator reserve and tolerance to hypothermic global ischemia (Yamamoto and Avkiran). 1993;105: 120-31

Selective pulmonary vasodilatation with inhaled nitric oxide (Tönz et al). 1993;105:760-2 (Letter)
Vasodilato: agents

Should adenosine continue to be ignored as a cardioprotective agent in cardiac operations? (Galiñanes et al). 1993;105:180-3 (Letter)

Vena cava, superior

Actuarial five-year survival estimates (Mengoli) (Letter); (Dartevelle and Macchiarini) (Reply). 1993;105:375-7

Ventilation, mechanical; see Respiration, artificial

Ventricle; see Heart ventricle

Ventricular assist system; see Heart-assist devices

Ventricular fibrillation

Determinants of myocardial oxygen consumption in fibrillating dog hearts: comparison between normothermia and hypothermia (Yaku et al). 1993;105:679-88

Ventricular function

Skeletal muscle-powered ventricle: effects of size and configuration on ventricular function (Oda et al). 1993;105:68-77

Ventricular function after normothermic versus hypothermic cardioplegia (Yau et al). 1993;105:833-44

Ventricular function, left

Acute isovolemic hemodilution and blood transfusion: effects on regional function and metabolism in myocardium with compromised coronary blood flow (Spahn et al). 1993;105:694-704

Annuloplasty with flexible or rigid ring does not alter left ventricular systolic performance, energetics, or ventricular-arterial coupling in conscious, closed-chest dogs (Castro et al). 1993; 105:643-59

Cardiac-derived thromboxane $A_{2}$ : An initiating mediator of reperfusion injury? (Byme et al). 1993;105:689-93

Left ventricular function in experimental mitral regurgitation with intact chordae tendineae (Hennein et al). 1993;105:624-32

Left ventricular pressure overload during postnatal development: effects on coronary vasodilator reserve and tolerance to hypothermic global ischemia (Yamamoto and Avkiran). 1993;105: 120-31

The protective effect of magnesium on acute catecholamine cardiotoxicity in the neonate (Caspi et al). 1993; 105:525-31

Ventricular function, right

Anatomic correction of atrioventricular discordance (Yamagishi et al). 1993;105:1067-76

Biventricular repair of hypoplastic right ventricle assisted by pulsatile bidirectional cavopulmonary anastomosis (Muster et al). 1993:105:112-9

Ventricular outflow tract obstruction

Is there an anatomic basis for subvalvular right ventricular outflow tract obstruction after an arterial switch repair for complete transposition? A morphometric study and review (Akiba et al). $1993 ; 105: 142-6$

Ventricular septal defects; see Heart septal defects, ventricular

Ventricular septum; see Heart septum

Video-assisted thoracoscopy; see Thoracoscopy

\section{w}

Warm blood cardioplegia; see Heart arrest, induced

Western Thoracic Surgical Association

Annual meeting notes. 1993;105:382

Notice of annual meeting. 1993;105:191-2, 380-1, 577-8, 771-2, 958-9, 1127-8

Program for annual meeting. 1993;105:960-4

Whites

Lengths of different routes for esophageal replacement in a white population (Rakić and Djuranović). 1993;105:1122 (Letter)

Whole blood; see Blood

Wounds, nonpenetrating

Blunt injury of the innominate artery: use of a vascular pericardial flap (Asimacopoulos et al). 1993;105:764-5 (Letter) 